\title{
(Bio)degradable and Biocompatible Nano- Objects from Polymerization-Induced and Crystallization-Driven Self-Assembly
}

\author{
Chen Zhu, Julien Nicolas*
}

Université Paris-Saclay, CNRS, Institut Galien Paris-Saclay, 92296 Châtenay-Malabry, France

*To whom correspondence should be addressed.

Email: julien.nicolas@u-psud.fr

Tel.: +33146835853 


\begin{abstract}
Polymerization-induced self-assembly (PISA) and crystallization-driven self-assembly (CDSA) techniques have emerged as powerful approaches to produce a broad range of advanced synthetic nano-objects with high potential in biomedical applications. PISA produces nano-objects of different morphologies (e.g., spheres, vesicles and worms), with high solids content ( $10-50 \mathrm{wt} . \%)$ and without additional surfactant. CDSA can finely control the self-assembly of block copolymers and readily forms non-spherical crystalline nano-objects and more complex, hierarchical assemblies, with spatial and dimensional control over particle length or surface area, which is typically difficult to achieve by PISA. Considering the importance of these two polymerization processes in the current scientific landscape of block copolymer self-assembly and the craze for their use in the biomedical field, this review will focus on the advances in PISA and CDSA to produce nano-objects suitable for biomedical applications in terms of (bio)degradability and biocompatibility. This review will therefore discuss these two aspects in order to guide the future design of block copolymer nanoparticles for future translation towards clinical applications.
\end{abstract}




\section{Introduction}

Over the past few decades, there has been a growing demand for the development of synthetic polymer nanoparticles for biological applications such as drug delivery. Polymer nanoparticles indeed offer key advantages over the use of free drugs. They improve the therapeutic index of drugs by ensuring a sustained drug release, they protect them from early metabolization and induce preferential accumulation in the diseased area, they result in reduced off-target toxicity and side effects. ${ }^{1}$ Biodegradability and/or biocompatibility are two essential properties to confer on polymeric nanoparticles to ensure their innocuousness once administered in the human body. Biodegradable polymers are polymers whose degradation is governed by biological activity (i.e., via cells), whereas degradable polymers is the preferred term for polymers that degrade hydrolytically in vitro or in vivo, or by the action of enzymes in vitro. ${ }^{2}$ As for biocompatible polymers, they should not produce toxic products and/or stimulate an immune response. ${ }^{3}$ A crucial point to bear in mind when developing new polymers for biomedical applications is that neither the starting polymers, nor their degradation products (if they are (bio)degradable) should be toxic to the surrounding environment. ${ }^{3}$ Among the different classes of biodegradable synthetic polymers that have been studied for biomedical applications, ${ }^{4}$ aliphatic polyesters (e.g., polylactide (PLA), poly(e-caprolactone) (PCL), poly(lactide-co-glycolide) (PLGA)), ${ }^{5}$ polypeptides (from $N$ carboxyanhydrides (NCAs) polymerization $)^{6}$ and poly(alkyl cyanoacrylates) ${ }^{7}$ are the most representative and some of them are even FDA-approved. When only biocompatibility is required,

well-established synthetic biocompatible polymers are poly(ethylene glycol) (PEG) ${ }^{8-12}$ PEGbased macromonomers, ${ }^{13}$ polysaccharides ${ }^{14}$ and some other hydrophilic polymers suggested as PEG alternatives. ${ }^{12,15}$

Biodegradable polymer nanoparticles are mostly obtained by formulation of preformed biodegradable polymers by nanoprecipitation/solvent displacement method or emulsion solvent evaporation, ${ }^{1,4,16,17}$ because of the difficulty/impossibility to perform their in situ synthesis in aqueous dispersed media. However, the resulting nanoparticles exhibit very low concentrations 
( 1-5 wt.\%), often use surfactants, and mostly lead to spherical morphologies, which may severely limit their use.

Polymerization-induced self-assembly (PISA) has recently emerged as one of the most robust polymerization processes capable of producing aqueous suspensions of polymer nano-objects of different morphologies (e.g., spheres, vesicles and worms), with high solids content ( 10-50 wt.\%) and without additional surfactant. ${ }^{18-21}$ It relies on the chain extension of a solvophilic macro initiator/chain transfer agent (macro-CTA) previously prepared by reversible-deactivation radical polymerization (RDRP), ${ }^{22-25}$ by a solvophilic/solvophobic monomer whose polymer is solvophobic. ${ }^{26,27}$ Self-assembly then occurs during the chain-extension, leading to different morphologies such as spheres, worms or vesicles. It is also worth noting its high versatility in terms of monomers, ${ }^{28,29}$ polymerization methods/reaction conditions, ${ }^{20}$ obtained morphologies, ${ }^{30}$ functionalities/features, ${ }^{31,32}$ as well as its potential use in biomedical applications. ${ }^{33,34}$

Crystallization-driven self-assembly (CDSA) is also another process that attracted tremendous attention due to the possibility to control the self-assembly of block polymers and to readily form non-spherical nanoparticles and more complex, hierarchical assemblies. ${ }^{35}$ It relies on the use of block copolymers with a semi-crystalline block, whose crystallization is promoted after introduction of a non-solvent to the crystalline block. It then drives self-assembly from spheres to 1D cylindrical structures and 2D platelet structures, ${ }^{36}$ with spatial and dimensional control over particle length or surface area, which is typically difficult to achieve by PISA. ${ }^{35,37,38}$ Such nanoassemblies have attracted considerable interest in many applications, including drug delivery, ${ }^{39,40}$ light-harvesting ${ }^{41,42}$ and optoelectronic materials. ${ }^{41}$

Considering the importance of these two polymerization processes in the current scientific landscape of block copolymer self-assembly and the craze for their use in the biomedical field, we thought it would be important to review the advances in PISA and CDSA to produce nano-objects suitable for biomedical applications in terms of (bio)degradability and biocompatibility. This review will therefore focus on discussing the biodegradability and biocompatibility aspect of block 
copolymer nano-objects obtained by the PISA and CDSA processes, as well as other aspects such as their morphologies and biomedical applications (Figure 1).

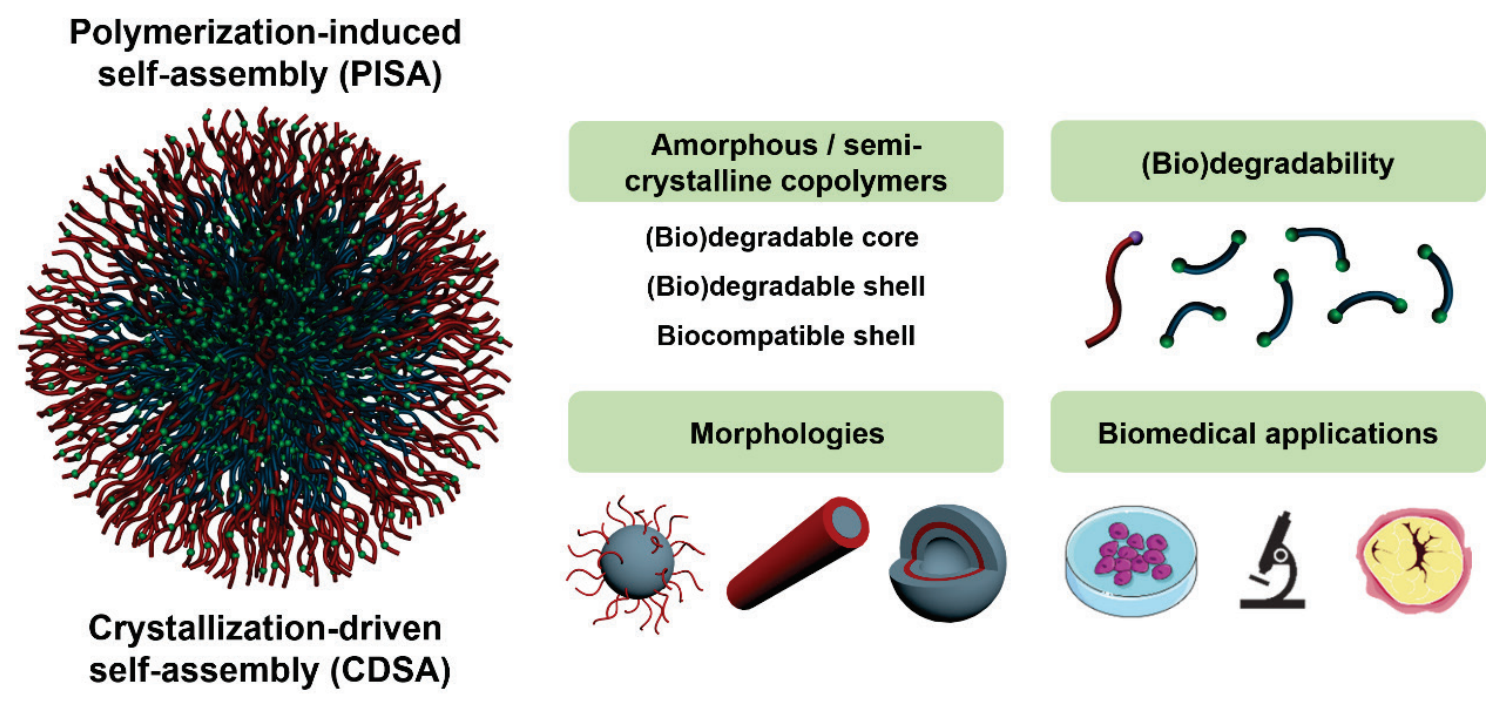

Figure 1. (Bio)degradable and/or biocompatible block copolymer nano-objects obtained by PISA or CDSA processes.

\section{Polymerization-induced self-assembly (PISA)}

\section{1. (Bio)degradable nano-objects by PISA}

\subsubsection{Ester-containing nano-objects}

Most PISA processes are based on the polymerization of vinyl monomers, resulting in nondegradable carbon-carbon backbones, which prevent their degradation and may induce toxic responses from the biological environment. Among the different approaches to confer degradability to vinyl polymers, radical ring-opening polymerization (rROP) of cyclic ketene acetals (CKA) offers a simple route towards multiple insertion of labile groups into the copolymer chain (Figure 2a). ${ }^{43-46}$ Indeed, when properly designed and/or employed in the right 
polymerization conditions, CKA monomers polymerize by a rROP mechanism and give ester groups in the copolymer backbone. They can then be hydrolysed and/or enzymatically cleaved, resulting in significant decrease in molar mass of the starting copolymer. ${ }^{44,45}$ While little progress has been made on the homopolymerization of CKA, their copolymerization with common vinyl monomers (e.g., methacrylates, acrylates, vinyl acetate, etc), by free-radical polymerization (FRP) or RDRP, has become a very productive area of research. ${ }^{45}$ Three main CKAs are currently widely used in copolymerization by rROP (Figure 2b): 2-methylene-1,3-dioxepane (MDO), 5,6-benzo-2methylene-1,3-dioxepane (BMDO) and 2-methylene-4-phenyl-1,3-dioxolane (MPDL).

(a)

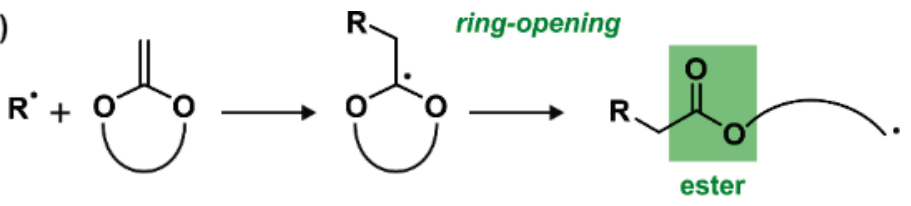

(b)
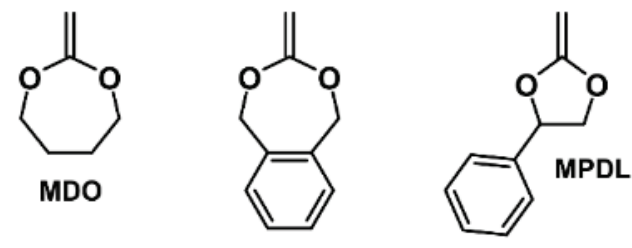

BMDO

Figure 2. (a) General mechanism for the rROP of cyclic ketene acetals (CKA). (b) Structures of 2methylene-1,3-dioxepane (MDO), 5,6-benzo-2-methylene-1,3-dioxepane (BMDO) and 2-methylene-4phenyl-1,3-dioxolane (MPDL). Reproduced from ref. ${ }^{45}$.

The first combination of PISA and rROP, naturally termed radical ring-opening (co)polymerization-induced self-assembly (rROPISA), was reported in $2019 .{ }^{47}$ It was performed in heptane to avoid early hydrolysis of CKA monomers while still conferring degradability to vinyl copolymer nanoparticles via incorporation of CKA units in the solvophobic block/nanoparticle core. $^{47}$ Based on a simple modification of a protocol reported by Armes, ${ }^{48}$ the CKA was copolymerized with benzyl methacrylate (BzMA) in heptane at $90{ }^{\circ} \mathrm{C}$ during chain extension of a 
poly(lauryl methacrylate) (PLMA) macro-CTA, that was previously synthesised by RAFTmediated polymerization (Figure 3). This approach was applied to MPDL, BMDO and MDO. ${ }^{49}$ Incorporation of CKAs did not really affect the PISA process, as stable nanoparticles were obtained comprising 4 to 40 mol.\% CKA monomer units in their solvophobic block. Interestingly, all nanoparticles exhibited spherical morphologies regardless the block length ratio and the fraction of CKA in the core, which was assigned by influence of RAFT agent and insertion of CKA. Even though degradation under accelerated conditions $(2.5 \mathrm{wt} \% \mathrm{KOH})$ was shown for the copolymers and films formed by solvent casting, the nanoparticle colloidal properties remained unchanged. 


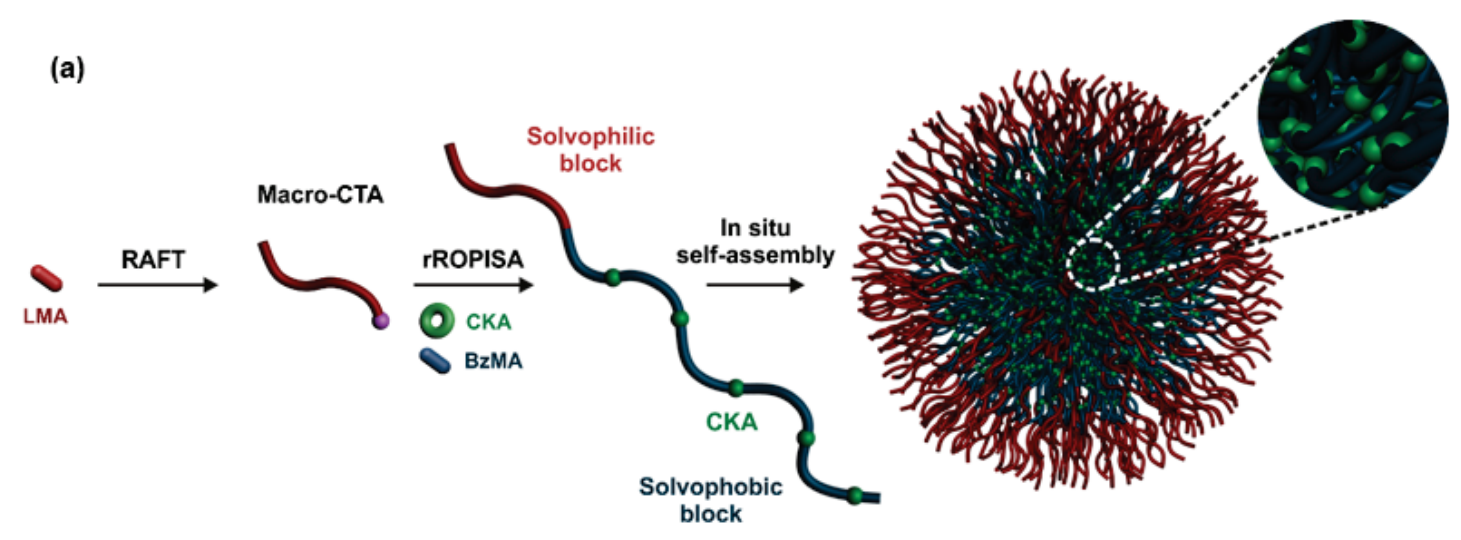

(b)

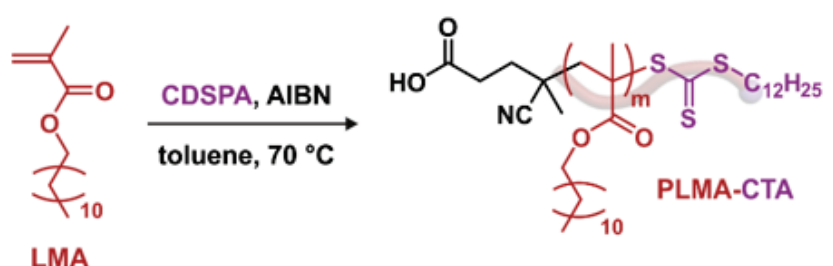

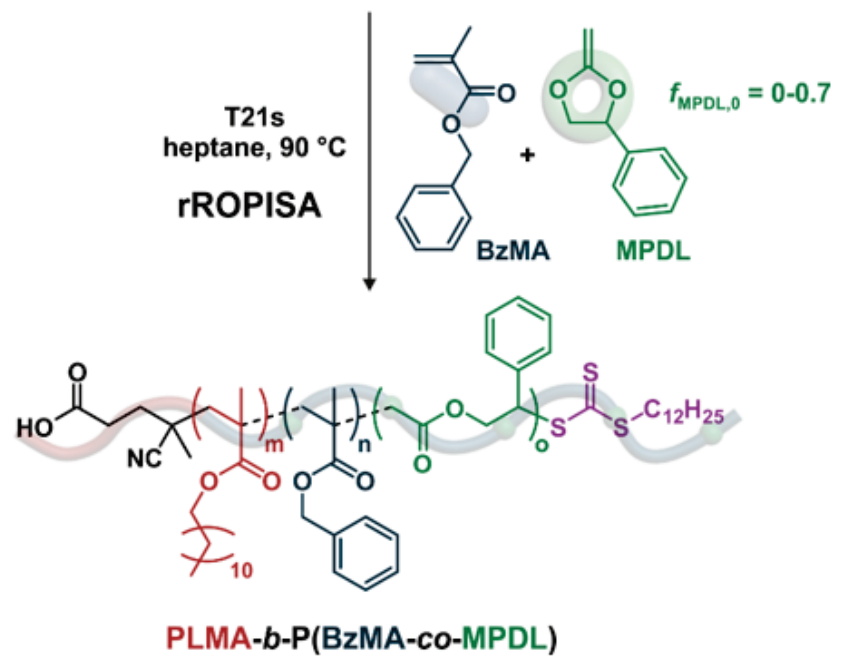

Figure 3. (a) Schematic representation of the rROPISA process for the synthesis of PLMA- $b-\mathrm{P}(\mathrm{BzMA}-\mathrm{co}-$ CKA) diblock copolymer nanoparticles. (b) RAFT-mediated synthesis of poly(lauryl methacrylate) (PLMA) followed by radical ring-opening copolymerization-induced self-assembly (rROPISA) of benzyl methacrylate (BzMA) and 2-methylene-4-phenyl-1,3-dioxolane (MPDL) to synthesize degradable PLMA$b$-P(BzMA-co-MPDL) diblock copolymer nanoparticles. Adapted from ref. ${ }^{47}$.

The rROPISA process was then extended to the synthesis of site-specific degradable nanoparticles by positioning the CKA either at the surface, or in the core, or at the surface and in the core of the nanoparticles (Figure 4). ${ }^{49}$ Inducing surface degradation was achieved by means of P(LMA-co- 
BMDO) and P(LMA-co-MPDL) macro-CTAs further chain-extended by BzMA under PISA conditions, whereas chain extension with BzMA in presence of CKA enabled the synthesis of "alldegradable" nanoparticles. Despite partial livingness of the macro-CTAs and a rather poor control of the polymerization during chain extension, stable nanospheres and porous nanospheres were formed for surface-degradable nanoparticles. A degradation study confirmed the presence of ester groups at the expected locations in the copolymers, with experimental molar masses that matched well the theoretical values after degradation.

(a)

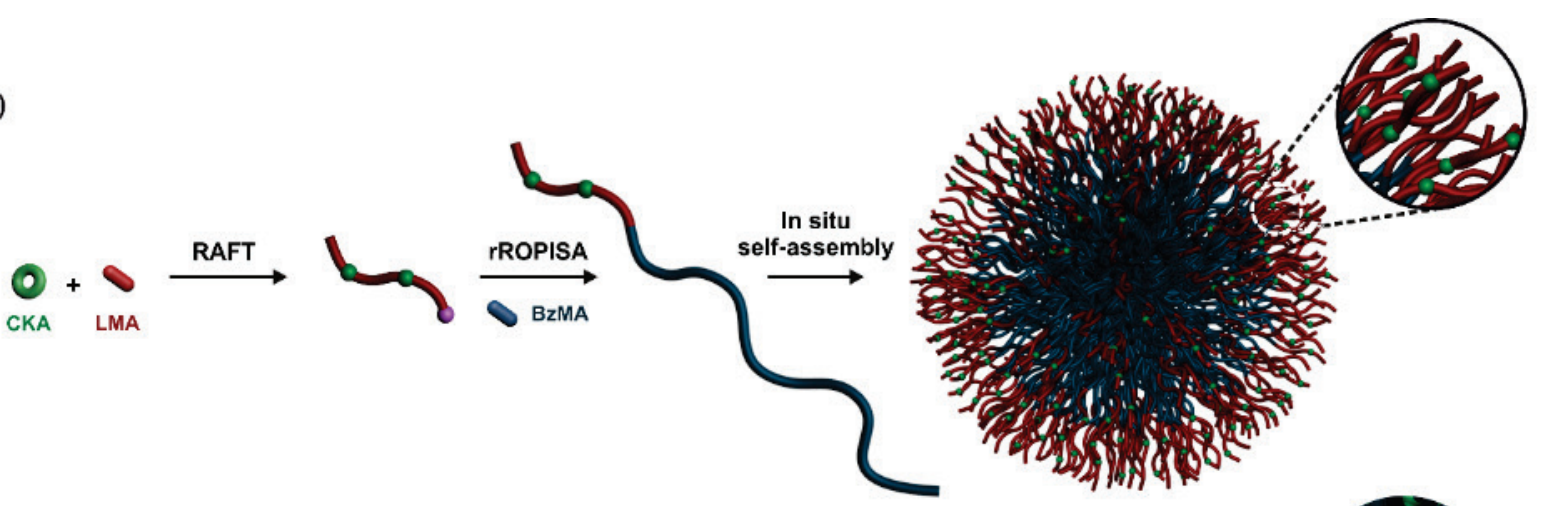

(b)

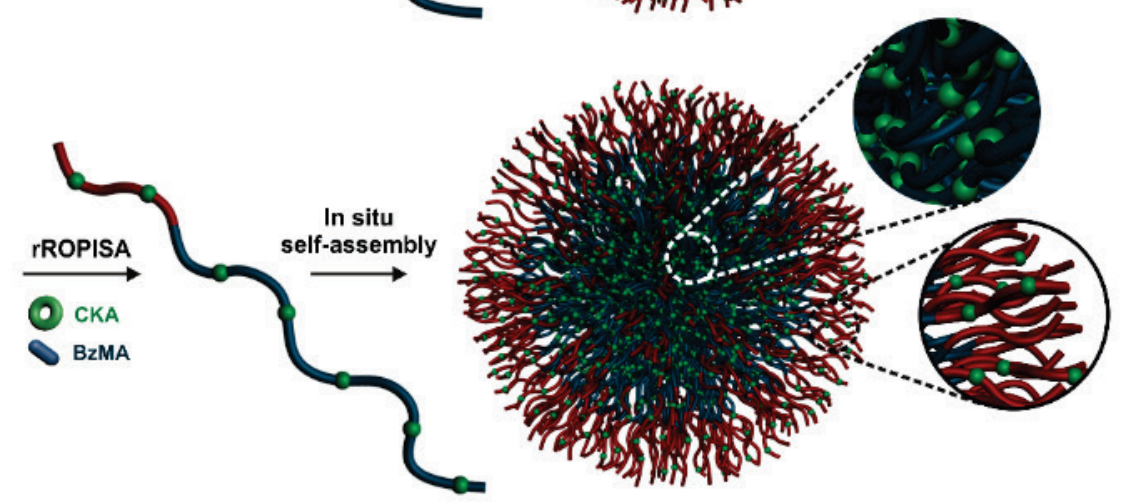

Figure 4. Radical ring-opening (co)polymerization-induced self-assembly (rROPISA) process for the synthesis of: (a) surface-degradable nanoparticles and (b) surface + core degradable nanoparticles using cyclic ketene acetals (CKAs) as ester bond precursors in the copolymer chains. Adapted from ref. ${ }^{49}$.

However, although this is a valuable proof of concept for the synthesis of degradable vinyl polymer nanoparticles by PISA, the main limitation of performing rROPISA in heptane is that the solvophilic shell of the nanoparticles prevents transfer to water to obtain aqueous suspensions of 
degradable copolymer nanoparticles. Also, rROPISA of CKA in aqueous dispersed media is very challenging because of the extreme sensitivity of CKA towards protic solvent and traces of water.

To overcome this limitation, a two-step rROPISA process was developed to allow the preparation of aqueous suspensions of CKA-containing polymer nanoparticles (Figure 5a). ${ }^{50}$ Poly[oligo(ethylene glycol) methyl ether methacrylate)]-b-poly(lauryl methacrylate-co-cyclic ketene acetal) (POEGMA- $b$-P(LMA-co-CKA)) diblock copolymer nanoparticles were first obtained in DMF, then transferred to water during which their colloidal properties were maintained thanks to a suitable PEG-based stabilizing shell (Figure 5b-d). This was successfully applied to the three main CKAs whose content could be adjusted, leading to cytocompatible nanoparticles on three different healthy cell lines that could be degraded in aqueous solution under accelerated conditions. Considering their PEG coating and the long-term hydrolytic degradation in physiological conditions of CKA-containing polymers, ${ }^{51}$ this study therefore allows to consider the use of nanoparticles obtained by rROPISA for drug delivery applications. 


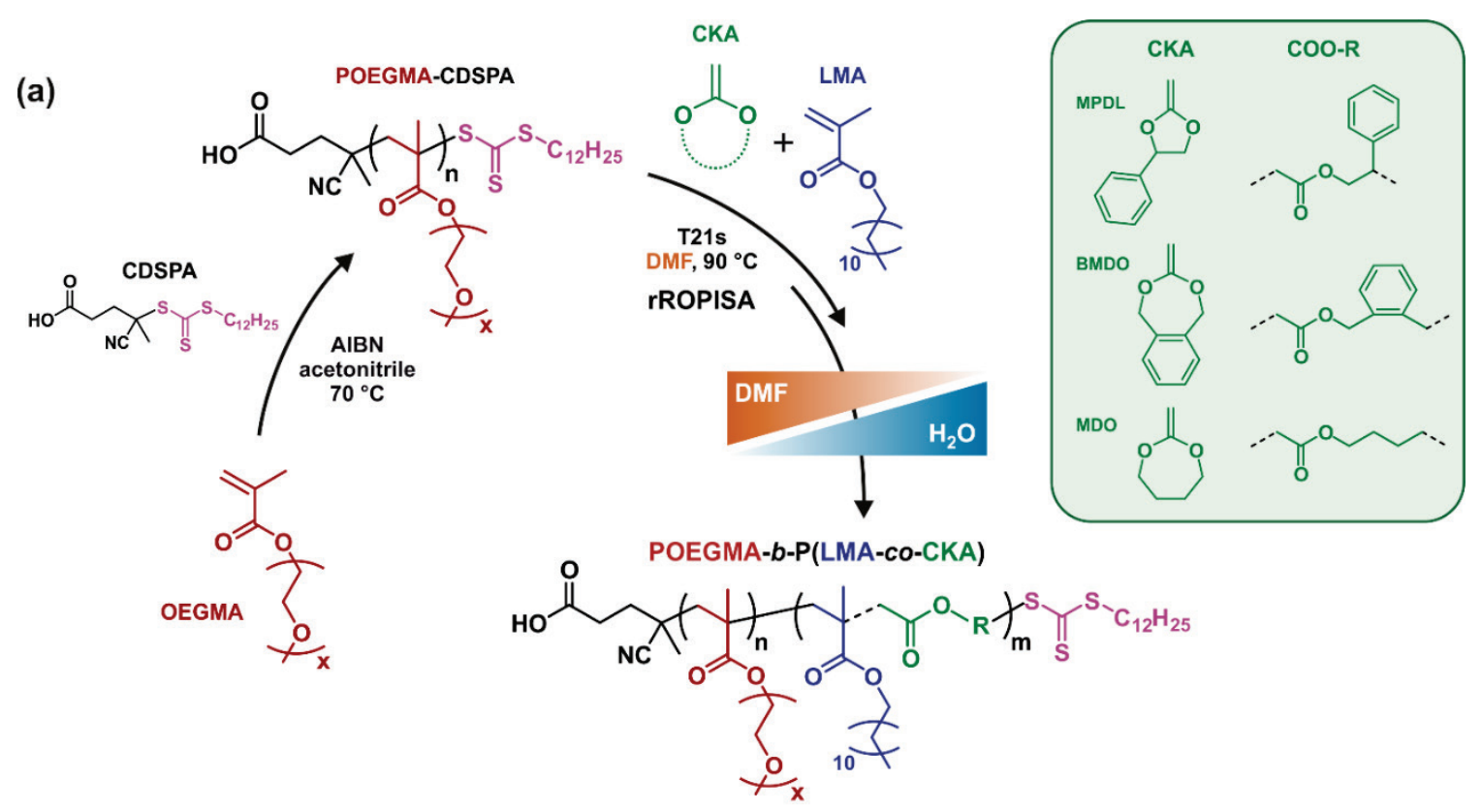

(b)

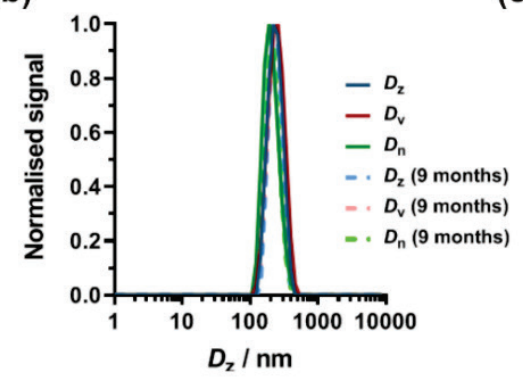

(c)

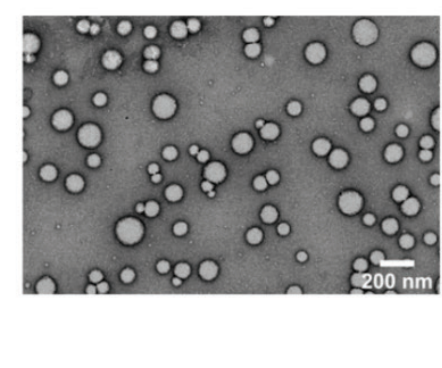

(d)

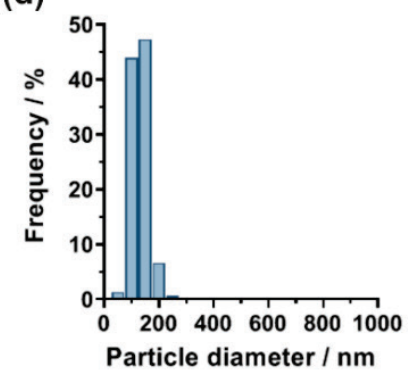

Figure 5. (a) 2-step rROPISA process to produce poly[oligo(ethylene glycol) methyl ether methacrylate)]$b$-poly(lauryl methacrylate-co-cyclic ketene acetal) (POEGMA-b-P(LMA-co-CKA)) diblock copolymer nanoparticles in DMF followed by a transfer to water to yield aqueous suspensions of degradable nanoparticles. (b) Average diameters from DLS reported in number $\left(D_{\mathrm{n}}\right)$, volume $\left(D_{\mathrm{v}}\right)$ and intensity $\left(D_{\mathrm{z}}\right)$ distribution after formulation and after 9 months, (c) representative TEM image and (d) particle size distribution by TEM of POEGMA- $b$-P(LMA-co-CKA) diblock copolymer nanoparticles $\left(F_{\mathrm{MPDL}}=0.09\right)$. Adapted from ref. ${ }^{50}$.

\subsubsection{Disulfide-containing nano-objects}

When switching from a CKA to a macrocyclic allylic sulfide monomer (3-methyl-idene-1,9-dioxa5,12,13-trithiacyclopentadecane-2,8-dione, MTC) as precursor of labile groups (e.g., disulfide, ester) into the copolymer backbone, stable aqueous suspensions of different nano-objects containing cleavable disulfide bonds in the solvophobic block were obtained by PISA (Figure 6). ${ }^{52}$ 
More specifically, a biocompatible poly(glycerol methacrylate) (PGMA) solvophilic macro-CTA was successfully chain extended with 2-hydroxypropyl methacrylate (HPMA) and MTC in water at $70^{\circ} \mathrm{C}$. Spheres, worms or vesicles were selectively obtained simply by adjusting the chain length of the core-forming block. Despite very low amounts of MTC $(<1 \mathrm{~mol} \%)$ were incorporated into the copolymer because of side reactions, disulfide bond cleavage was shown by subjecting the nanoparticles to a reductive environment using tris(2-carboxyethyl) phosphine (TCEP) at pH 8-9, resulting in a shape transformation from worm-like nanoparticles to spheres as demonstrated by SEC, TEM and DLS.

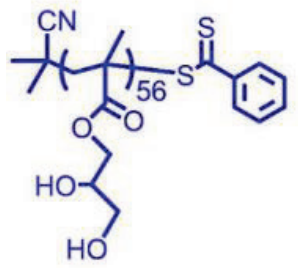

PGMA $_{56}$

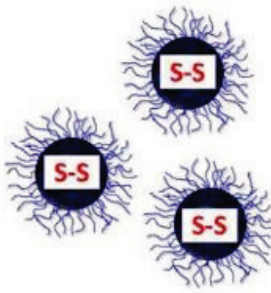

Spheres
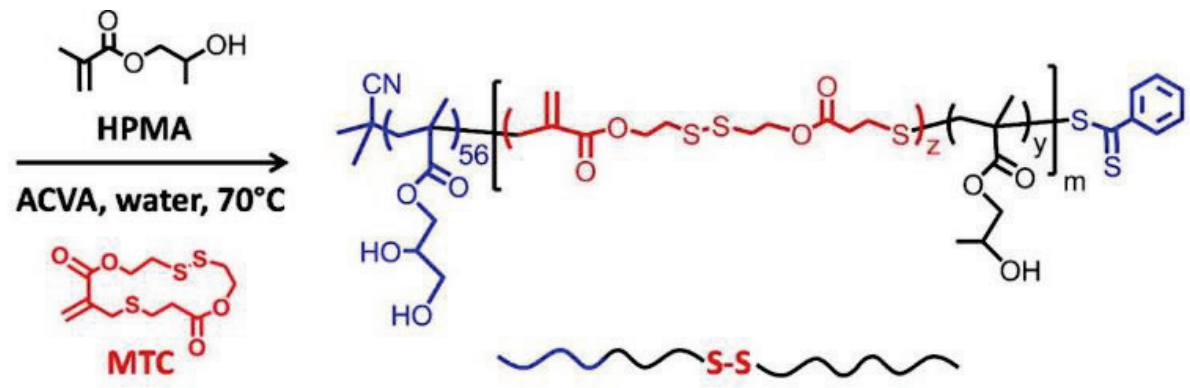

Polymerization-Induced Self-Assembly (PISA)

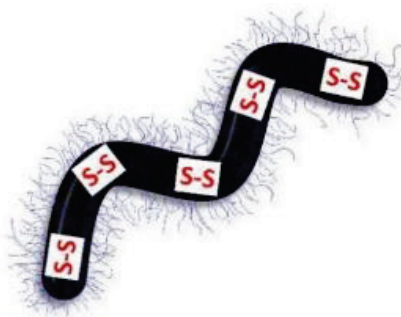

Worms

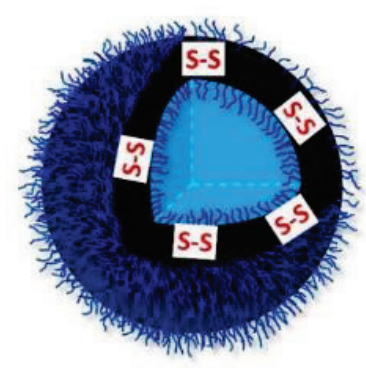

Vesicles

Increasing DP $(\mathbf{Y}+\mathbf{Z})$

Figure 6. Synthesis of $\mathrm{PGMA}_{56}-b-\mathrm{P}\left(\mathrm{HPMA}_{\mathrm{y}}-\mathrm{co}-\mathrm{MTC}_{\mathrm{z}}\right)$ diblock copolymer nano-objects (spheres, worms and vesicles as the overall target DP $(\mathrm{y}+\mathrm{z})$ is increased) via RAFT-mediated copolymerization of HPMA with MTC in aqueous solution at $70^{\circ} \mathrm{C}$. The nano-objects contain cleavable disulfide bonds in the hydrophobic P(HPMA-co-MTC) chains. Adapted from ref. ${ }^{52}$. 


\subsubsection{Synthetic polypeptide nano-objects}

Synthetic polypeptides are also promising polymers because of their biocompatibility and biodegradability. One of the most robust and versatile methods to produce synthetic polypeptides relies on the ROP of $N$-carboxyanhydrides (NCA). ${ }^{6}$ This approach often provides polypeptides with good control over the molecular weight, but it suffers from the high sensitivity of NCA monomers to water and moisture, and thus needs to operate in organic solvents. ${ }^{6}$ For instance, NCA-based ROPISA was performed in THF using L-phenylalanine (L-Phe) NCA (Figure 7). ${ }^{53}$ The one-pot polymerization was initiated by an amino poly(ethylene glycol) methyl ether under air at $10^{\circ} \mathrm{C}$ to yield PEG- $b$-P(L-Phe) polypeptides. Nanospheres or hollow vesicles were formed depending on initiator/monomer/solids content ratio. The degradation of nanoparticles was confirmed by enzymatic cleavage (using trypsin for $96 \mathrm{~h}$ ) of the vesicles after their transfer from THF to water, as evidenced by DLS and TEM.

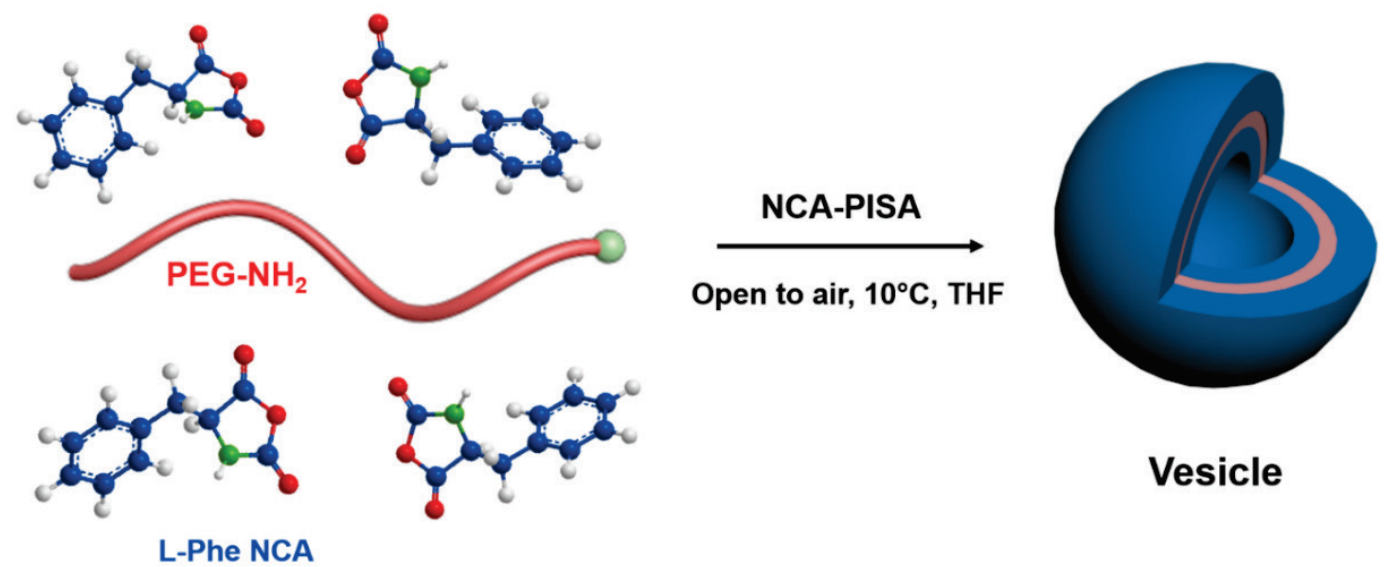

Figure 7. ROP of L-phenylalanine $N$-carboxyanhydrides (L-Phe NCA) in THF. Adapted from ref. ${ }^{53}$.

The first aqueous ROPISA of NCA was a major step forward in the synthesis of biodegradable nanoparticles (Figure 8). ${ }^{54}$ An $\alpha$-amino-poly(ethylene oxide) macroinitiator $\left(\mathrm{PEG}_{5 \mathrm{k}}-\mathrm{NH}_{2}\right)$ was chain extended with $\gamma$-benzyl-L-glutamate $N$-carboxyanhydrides ( $\gamma$-BLG NCA) in a sodium bicarbonate aqueous solution $(\mathrm{pH}=8.5,50 \mathrm{mM})$ under $4^{\circ} \mathrm{C}$ to protect $\mathrm{NCA}$ monomers from 
hydrolysis, resulting in well-defined amphiphilic diblock copolymer nano-objects with worm-like or needle-like morphologies at solids content up to $13 \mathrm{wt} \%$.

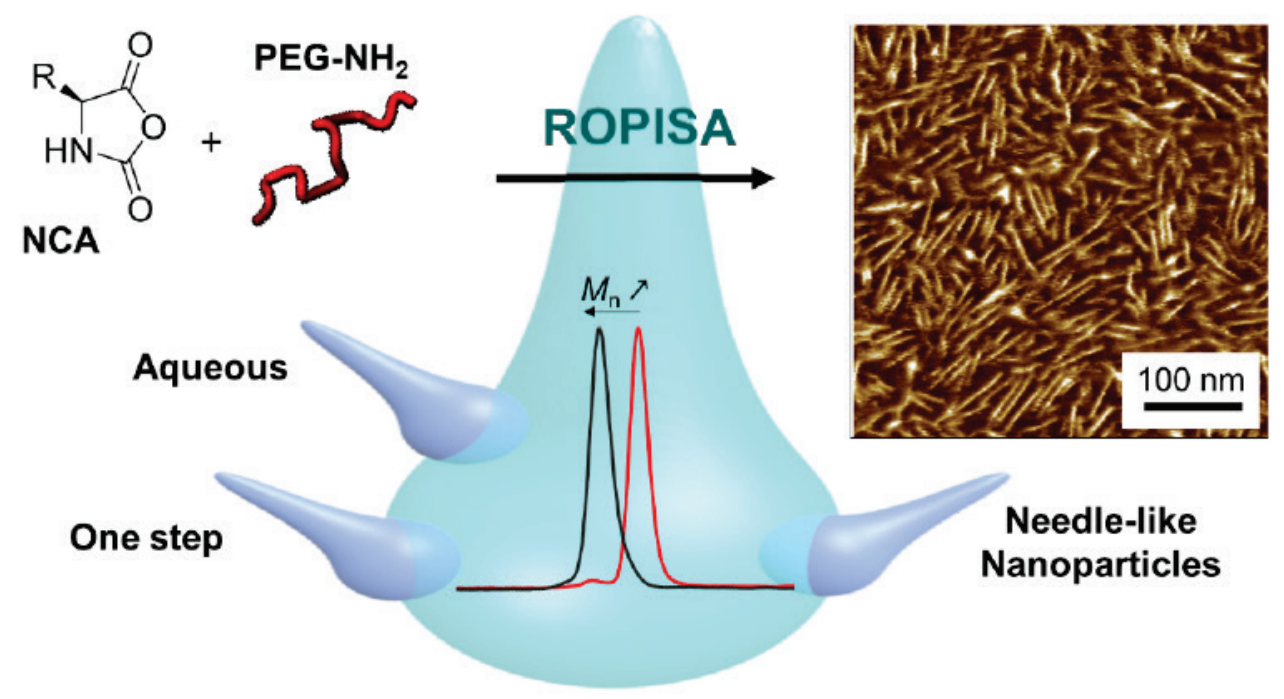

Figure 8. One-step ring-opening polymerization-induced self-assembly (ROPISA) of $N$ carboxyanhydrides (NCAs) initiated by a $\alpha$-amino-poly(ethylene glycol) macroinitiator for the synthesis of needle-like polypeptide nanoparticles. Adapted from ref. ${ }^{54}$.

\subsubsection{Polypeptoid nano-objects}

Polypeptoids are pseudo-peptidic polymers composed of an aliphatic polyamide backbone with substitution on the nitrogen atoms (Figure 9). ${ }^{55}$ Like synthetic polypeptides, their potential degradability, non-cytotoxicity and non-immunogenicity make them promising candidates for biomedical applications. Conversely to polypeptides, such unique $N$-substituted polyglycine backbones induce random coil conformations or well-defined secondary structures. It has been shown that polypeptoids can be degraded hydrolytically under accelerated conditions whereas degradation is very slow in a cellular environment. ${ }^{56}$ Also, such polymers exhibit enhanced resistance to enzymatic degradation. ${ }^{56}$ 


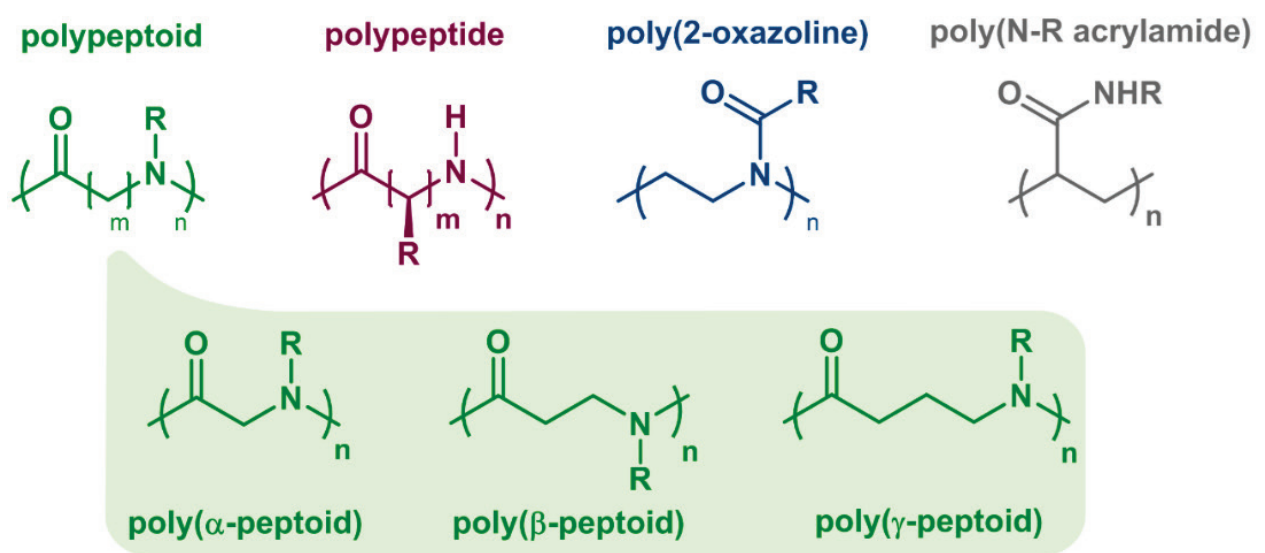

Figure 9. Chemical structures of polypeptides and pseudo-peptidic polymers. Adapted from ref. ${ }^{55}$.

Poly(sarcosine) (PSar) is a well-known polypeptoid, whose monomer (Sar NCA) is similar to alanine but with the methyl residue bound to the nitrogen. ${ }^{57}$ PSar exhibits similar "stealth" properties as PEG, making it suitable candidate to design long circulating nanoparticle. ${ }^{15,58,59}$ PSar was used as a macro-CTA to generate PSar-based nano-objects by photo-polymerization-induced self-assembly (photo-PISA), in order to investigate their resistance to enzymatic degradation. ${ }^{60}$ PSar was synthesized by ROP of Sar NCA followed by conjugation to a trithiocarbonate RAFT agent to allow the photo-PISA of HPMA in water (Figure 10a). Different morphologies were obtained depending on the solids content and chain length of the PHPMA block (Figure 10b). In particular, unilamellar PSar- $b$-PHPMA vesicles were studied for their proteolytic resistance ability against enzymes (e.g., $\alpha$-chymotrypsin, trypsin, and pepsin). Both empty and enzyme-loaded vesicles presented superior stability in the presence of proteolytic enzymes, demonstrating their resistance to enzyme degradation. 
a)

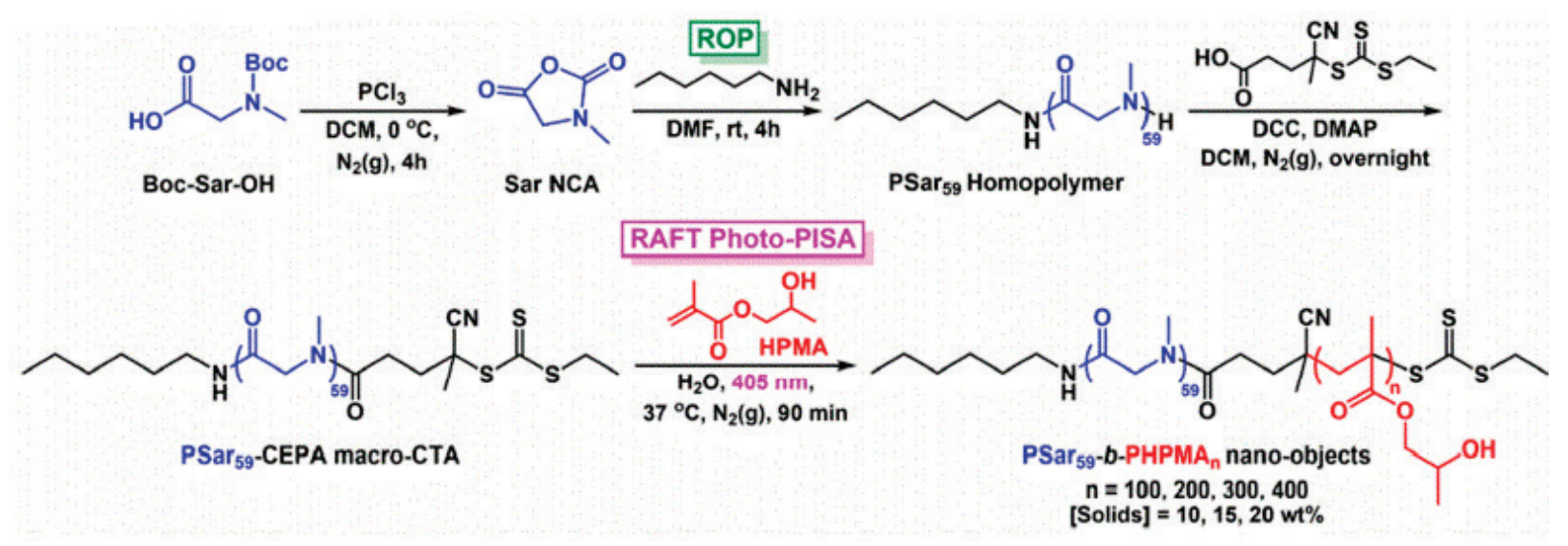

b)
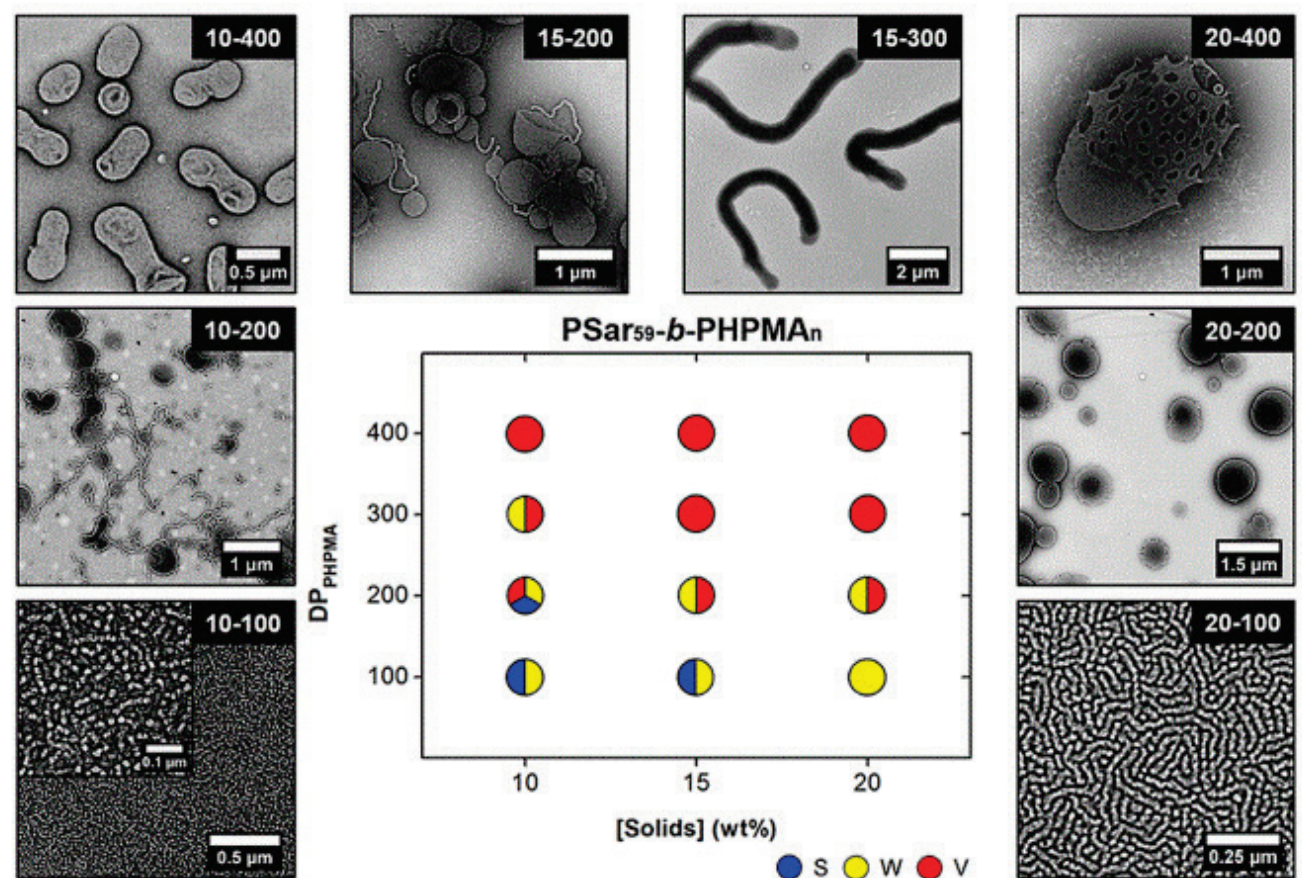

Figure 10. (a) Synthesis of PSar- $b$-PHPMA diblock copolymers by aqueous photo-PISA. (b) Phase diagram of PSar $59-b$-PHPMA $(\mathrm{n}=100-400)$ diblock copolymer nano-objects prepared via aqueous photo-PISA by adjusting the solids content and the PHPMA chain length (blue circles $=$ spheres, yellow circles $=$ worms, red circles $=$ vesicles). Adapted from ref. ${ }^{60}$. 


\subsection{Biocompatible nano-objects by PISA}

Biocompatibility is a key parameter when designing (polymer) materials for biomedical applications. This is an even more important parameter to consider when developing new nanoparticulate systems for drug delivery. In this context, if biodegradability cannot be implemented, it is therefore important that PISA-derived nano-objects are made at least of biocompatible polymer building blocks. This is true especially at the level of the shell, as those polymers would be mainly responsible for the interaction with the biological medium once in the body, thus governing the fate of the nanoparticles. ${ }^{61}$ In practice, in addition to natural polymers like proteins/polypeptides, oligonucleotides and polysaccharides, synthetic polymers such as poly(ethylene glycol) (PEG), poly(2-hydroxypropyl) methacrylamide (PHPMAAm), poly(vinyl pyrrolidone) (PVP), poly(2-oxazoline)s, polypeptoids, polyacrylamides and a few other polymers have generated solid data in terms of biocompatibility, and some of them have also been safely used in clinical trials. ${ }^{9}$ Here we summarize the results obtained by PISA with the main natural and synthetic biocompatible polymers as solvophilic block, which were therefore used as shell-forming polymers (Figure 11). 
(a)
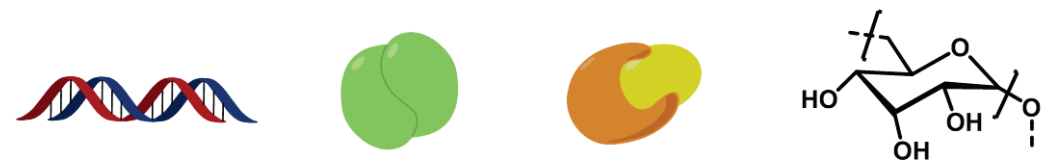

DNA

Protein

Enzyme

Polysaccharide

(b)

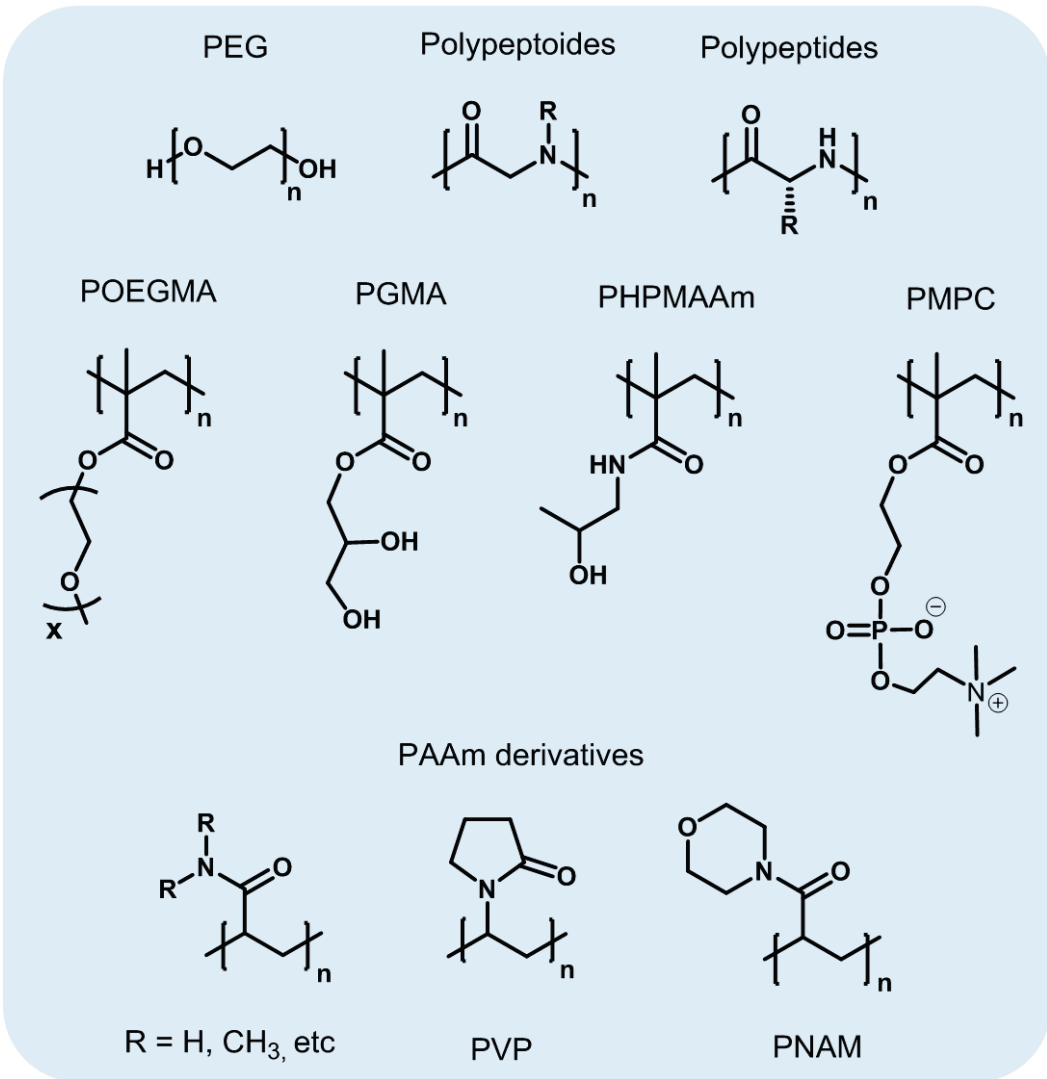

Figure 11. Schematic representation or structures of: (a) natural and (b) synthetic biocompatible polymers used as shell-forming block in PISA. PEG = poly(ethylene glycol), PAAm = poly(acrylamide), POEGMA = poly[oligo (ethylene glycol) methacrylate], PGMA = poly (glycerol methacrylate), PHPMAAm = poly $(2-$ hydroxypropyl methacrylamide), PMPC = poly[2-(methacryloyloxy) ethyl phosphorylcholine], PVP = poly(vinyl pyrrolidone), PNAM = poly $(N$-acryloylmorpholine $)$.

\subsubsection{Poly(ethylene glycol)-based polymers}

As seen again recently with the development of mRNA vaccines, PEG is so far the most widely used hydrophilic polymer for biological applications due to its stabilizing and stealth properties. ${ }^{8,10,12,15,62}$ Many PEGylated therapies or drug-loaded nanoparticles have been developed and have either reached the stage of clinical trials or even been approved by the FDA. ${ }^{5}$ Despite 
some concerns regarding PEG-related hypersensitivity, its non-degradability or its potential accumulation in the body, ${ }^{12}$ PEG is still the golden standard in the biomedical field when considering biocompatible hydrophilic polymers, which explains why PEG-based polymers have been extensively used as solvophilic macro-CTA in PISA.

In this regard, the synthesis of amphiphilic PEG- $b$-PHPMA diblock copolymers represents an interesting combination, as PHPMA is often used as hydrophobic polymer for the design of nanocarriers for biomedical applications. The first example of PEG- $b$-PHPMA copolymer nanoparticles formed by aqueous PISA was achieved from a PEG $_{113}$ macro-CTA that was chain extended with HPMA at $70^{\circ} \mathrm{C} .{ }^{63}$ Different morphologies like spheres, worms or vesicles were targeted by varying the chain length of core-forming block or the solids content. This copolymer has shown promising results in terms of encapsulation of macromolecules, for instance with bovine serum albumin (BSA) used as model biomacromolecule. ${ }^{64}$ The PISA process was conducted under light at room temperature and nearly complete conversion was achieved within $15 \mathrm{~min}$ at $405 \mathrm{~nm}$ irradiation. The successful encapsulation into the formed vesicles of BSA (35\% active) was shown by via Bradford assay. Given those promising results, encapsulation of enzymes, ${ }^{65-69}$ hydrophilic ${ }^{64,70}$ and hydrophobic ${ }^{71}$ proteins, contrast agents ${ }^{72}$ and other molecules ${ }^{73}$ have also been reported (Table 1). 
Table 1. Encapsulation of Biomacromolecules and Other Payloads by PISA During the Synthesis of PEG$b$-PHPMA, POEGMA- $b$-PHPMA and PGMA- $b$-PHPMA Diblock Copolymer Nanoparticles.

\begin{tabular}{|c|c|c|c|c|c|}
\hline \multicolumn{2}{|c|}{ Payloads $^{a}$} & Method & $\begin{array}{l}\text { Hydrophilic } \\
\text { block }\end{array}$ & Applications & Ref \\
\hline \multirow{6}{*}{ Enzymes } & \multirow{3}{*}{ HRP } & $\begin{array}{l}\text { In situ } \\
\text { encapsulation }\end{array}$ & $\mathrm{PEG}_{113}$ & Enzymatic nanoreactor & 67 \\
\hline & & $\begin{array}{c}\text { In situ } \\
\text { encapsulation }\end{array}$ & $\mathrm{PGMA}_{40}$ & Enzymatic nanoreactor & 69 \\
\hline & & $\begin{array}{c}\text { Physical } \\
\text { encapsulation }\end{array}$ & $\mathrm{PEG}_{113}$ & $\begin{array}{l}\text { Thermo-responsive } \\
\text { enzymatic nanoreactor }\end{array}$ & 66 \\
\hline & \multirow{2}{*}{ Gox } & $\begin{array}{c}\text { In situ } \\
\text { encapsulation }\end{array}$ & $\mathrm{PEG}_{113}$ & Enzymatic nanoreactor & 67 \\
\hline & & $\begin{array}{c}\text { In situ } \\
\text { encapsulation }\end{array}$ & $\mathrm{PEG}_{113}$ & Antimicrobial property & 68 \\
\hline & ASNS & $\begin{array}{c}\text { In situ } \\
\text { encapsulation }\end{array}$ & $\mathrm{PEG}_{113}$ & Carrier & 65 \\
\hline \multirow{5}{*}{ Proteins } & \multirow{2}{*}{ BSA } & \multirow{2}{*}{$\begin{array}{c}\text { Physical } \\
\text { encapsulation } \\
\text { In situ } \\
\text { encapsulation }\end{array}$} & $\mathrm{PEG}_{113}$ & Carrier & 64 \\
\hline & & & $\mathrm{PGMA}_{40}$ & Enzymatic nanoreactor & 69 \\
\hline & GFP & $\begin{array}{c}\text { In situ } \\
\text { encapsulation }\end{array}$ & $\mathrm{PEG}_{113}$ & Enzymatic nanoreactor & 67 \\
\hline & $\mathrm{OmpF}$ & $\begin{array}{c}\text { In situ } \\
\text { encapsulation }\end{array}$ & $\mathrm{PEG}_{113}$ & $\begin{array}{l}\text { Channel-forming membrane } \\
\text { with hydrophobic protein }\end{array}$ & 71 \\
\hline & IFN & Grafting from & POEGMA & Protein delivery/conjugation & 70 \\
\hline $\begin{array}{l}\text { Contrast } \\
\text { agents }\end{array}$ & Iohexol & Graft through & $\mathrm{PEG}_{111}$ & $\mathrm{X}$-ray image & 72 \\
\hline \multirow{3}{*}{ Others } & Silica & $\begin{array}{c}\text { Physical } \\
\text { encapsulation }\end{array}$ & $\mathrm{PEG}_{113}$ & Carrier & 64 \\
\hline & $\begin{array}{c}\mathrm{Au} \\
\text { nanoparticles }\end{array}$ & $\begin{array}{l}\text { Host-guest } \\
\text { interaction }\end{array}$ & $\begin{array}{c}\beta-C D- \\
\text { POEGMA }_{13}\end{array}$ & $\begin{array}{l}\text { Polymer-based } \\
\text { nanocomposites }\end{array}$ & 73 \\
\hline & Rho & $\begin{array}{l}\text { Cross-linking } \\
\text { encapsulation }\end{array}$ & $\mathrm{PEG}_{102}$ & Carrier & 74 \\
\hline
\end{tabular}

${ }^{a} \mathrm{HRP}=$ horseradish peroxidase Gox = glucose oxidase; ASNS = L-asparaginase; BSA = bovine serum albumin; GFP $=$ green fluorescent protein; $\mathrm{OmpF}=$ outer membrane protein $\mathrm{F} ; \mathrm{IFN}=$ interferon- $\alpha$; Rho $=$ Rhodamine $\mathrm{B}$. 
To study the potential of PEG- $b$-PHPMA nanoparticles as enzymatic nanoreactors, a photoinitiated aqueous PISA system was performed using visible light without initiator at $37^{\circ} \mathrm{C}$ to form nanoparticles with different morphologies (Figure 12). ${ }^{67}$ Horseradish peroxidase (HRP) and glucose oxidase (Gox) were selected as enzymes to be encapsulated into the nanoparticles during the PISA process. HRP catalyzes the oxidation of 3,3'-dimethoxybenzidine (DMB) into a colored dimer product, whereas Gox catalyzes the oxidation of $D$-glucose into $\delta$-glucono-1,5-lactone and produces hydrogen peroxide $\left(\mathrm{H}_{2} \mathrm{O}_{2}\right)$ as a byproduct. HRP and Gox enzymes were encapsulated insitu to form enzyme-loaded vesicles. The enzyme loading efficiencies were in the $27-54 \%$ range, with enzyme activity approaching $60 \%$ for both enzymes. An enzymatic cascade reaction was also performed to further illustrate the efficiency of the system by mixing vesicles loaded with each enzyme in a 1:1 molar ratio in PBS, resulting in an overall enzyme activity of $46 \%$. The encapsulation of green fluorescent protein (GFP) into the same system demonstrated the compartmentalization and retention of GFP inside the vesicles' lumens. Despite a loss in fluorescence after purification of the vesicles, $56 \%$ of the GFP was retained with $11 \%$ encapsulation efficiency. 

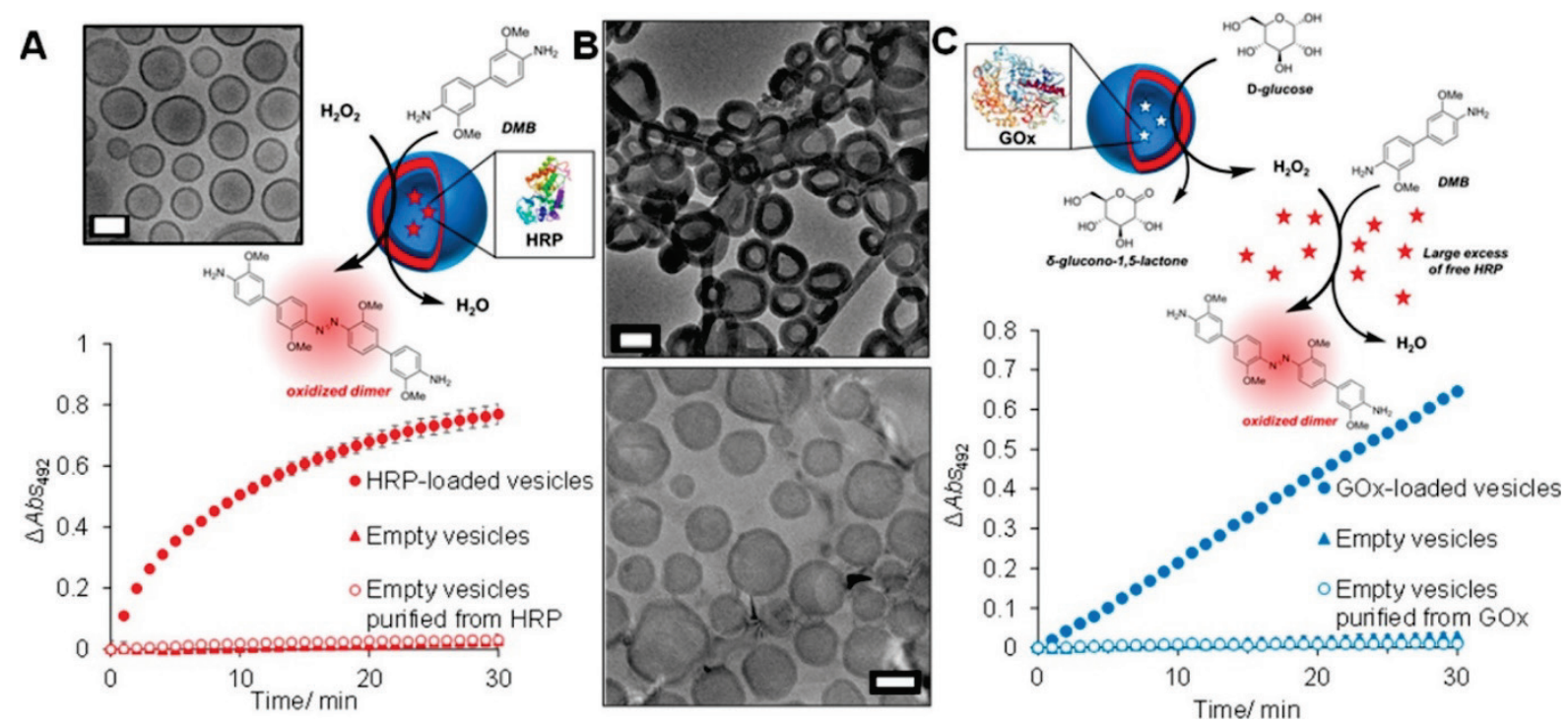

Figure 12. (A) Cryo-TEM image of HRP-loaded vesicles and schematic showing the HRP-catalyzed oxidation of DMB to its coloured dimer product detected in the colorimetric assay, as well as activity of the HRP-loaded vesicles against negative controls. (B) Cryo-TEM images of Gox-loaded vesicles using commercial Gox (top) and purified Gox (bottom). (C) Schematic showing the Gox-catalyzed oxidation of d-glucose to $\delta$-glucono-1,5-lactone, as well as activity of the Gox-loaded vesicles against negative controls. Scale bars $=200 \mathrm{~nm}$. Adapted from ref. ${ }^{67}$.

The enzyme activity could also be controlled by the use of thermo-responsive nanoparticles obtained by PISA. ${ }^{66}$ In this context, PEG $_{113}-b$-PHPMA 400 nanoparticles were used as seeds for subsequent copolymerization with $N$-isopropylacrylamide (NIPAM) and allyl acrylamide (ALAM) by light-initiated PISA. The HRP enzyme was loaded during the crosslinking process with a loading efficiency of $\sim 28 \%$. HRP activity was controlled by adjusting the temperature of $\mathrm{PEG}_{113^{-}}$ $b$-PHPMA $400-b$-P(NIPAM $\left.200-c o-\mathrm{ALAM}_{9}\right)$ thermoresponsive vesicles, which resulted in an increase in enzymatic reaction rate up to $32^{\circ} \mathrm{C}$, whereas a sharp decrease was observed when further increasing the temperature. It also indicates that hydrophobicity of the vesicular membrane directly affects the permeability to small molecules as they diffuse into the vesicles. To allow the release of bimacromolecules from the vesicles under mild conditions, non-cross-linked vesicles that can transform into worms at low temperature were also prepared by this route. 
The antimicrobial properties of Gox-loaded PEG $_{113}-b$-PHPMA nanoreactors were also demonstrated in response to glucose ${ }^{68}$ Colorimetric assays of enzymatic activity on Gox-loaded vesicles indicated high efficacy up to seven-log reduction in the growth of Gram-positive $(S$. aureus and S. epidermidis in Mueller-Hinton broth) and Gram-negative (E. coli and Klebsiella pneumoniae) bacteria at high glucose concentrations (5170 mg. $\left.\mathrm{L}^{-1}\right)$, with a five-log reduction achievable for Gram-positive bacteria at low glucose concentrations (170 mg. $\left.\mathrm{L}^{-1}\right)$. Their activity against resistant bacterial stains, such as a clinical isolate of methicillin-resistant S. aureus (MRSA), was also proven. Further optimizations in terms of nanoreactor concentrations was performed to be non-toxic against Gram-positive bacteria under physiological blood glucose concentrations.

Vesicles obtained from aqueous PISA can also find application as an alternative to the PEGylation of enzymes. ${ }^{65}$ This strategy yielded vesicles with a PHPMA membrane exhibiting size-selective permeability, allowing the loaded L-asparaginase (ASNS), used for leukemia treatment, to retain $52 \%$ of its activity and catalyze the removal of asparagine from the external medium (Figure 13). Such vesicles increased the proteolytic stability in vitro and in vivo, and reduced antibody recognition compared with the use of the free enzyme or the PEGylated counterpart. Furthermore, the ASNS-loaded vesicles inhibited cell proliferation on ASNS gene silenced A549 in vitro, with similar efficacy than the PEGylated ASNS bioconjugate (58\% vs. $61 \%)$. 


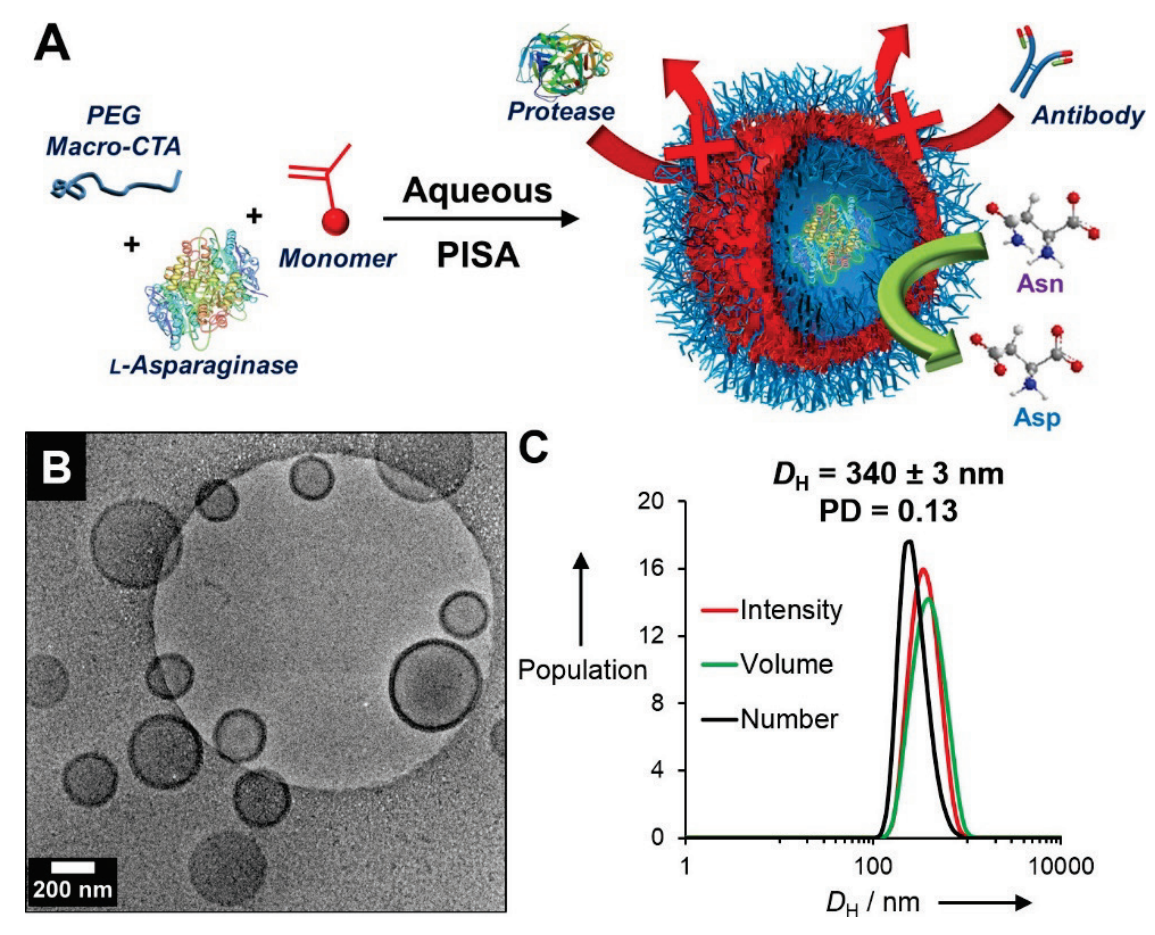

Figure 13. (A) Synthesis of ASNS-loaded vesicle by aqueous polymerization-induced self-assembly (PISA) with a semipermeable membrane that acts as a size-selective barrier allowing for therapeutic function, but not degradation or antibody binding. (B) Representative cryo-TEM image and (C) DLS size distribution of purified ASNS-loaded vesicles. Adapted from ref. ${ }^{65}$.

While encapsulation via PISA of hydrophilic proteins, such as BSA or GFP, has been reported, encapsulation of hydrophobic biomacromolecules is much less common. Investigation of the influence of small molecular surfactants on the targeted vesicular morphology showed the high tolerance of the aqueous photo-PISA process towards non-ionic surfactants conversely to ionic ones (Figure 14). Interestingly, a hydrophobic channel-forming outer membrane protein $\mathrm{F}(\mathrm{OmpF})$ porin was successfully inserted into the membrane of the vesicles with the assistance of non-ionic surfactants. $^{71}$ As a proof of concept, HRP was encapsulated into the lumen of the OmpF-containing vesicles, resulting in a $24 \%$ permeability enhancement compared OmpF-free HRP-loaded vesicles. This approach could therefore be of high interest for the design of biomimetic nanoreactors. 


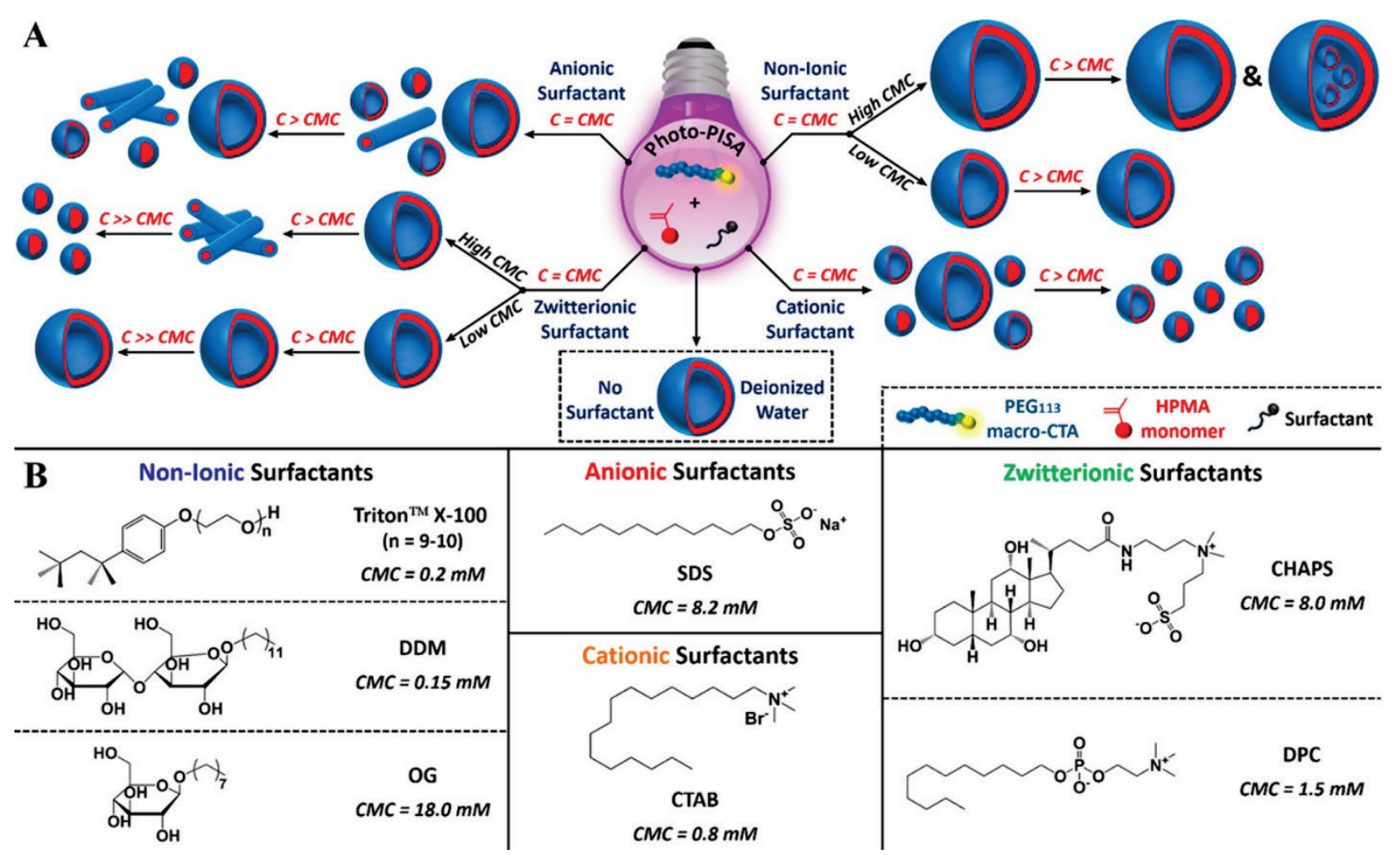

Figure 14. (A) Schematic illustration showing $\mathrm{PEG}_{113}-b-\mathrm{PHPMA}_{400}$ nano-object morphologies after aqueous photo-PISA in the presence of different surfactants. (B) Chemical structures and main characteristics of the surfactants used. Adapted from ref. ${ }^{71}$.

Encapsulation of fluorescent dyes or contrast agents in PEG- $b$-PHPMA copolymer nano-objects from PISA was also reported (Figure 15). To enlarge the imaging window for diagnosis, iohexol, a common contrast agent used in X-ray computed tomography (CT), was successfully incorporated into such nanoparticles through a cross-linking approach. ${ }^{72}$ Iohexol was turned into a hexa-acrylate crosslinker before its copolymerization with HPMA from a $\mathrm{PEG}_{111}$ macro-CTA in water at $70^{\circ} \mathrm{C}$. The resulting copolymer nanoparticles exhibited high colloidal stability in PBS and FBS for $48 \mathrm{~h}$, a rather high concentration (120 mg.mL $\left.\mathrm{m}^{-1}\right)$, and a high loading efficiency in iohexol (36.5\%), which ensure sufficient contrast in CT imaging. Compared to free iohexol, iohexol-loaded nanoparticles exhibit longer circulation time, lack of toxicity, similar imaging ability, and significant 
accumulation in tumors. However, further improvements are needed with respect to the lack of biodegradation and the limited amount of iodine that is still too low to meet clinical requirement.

A

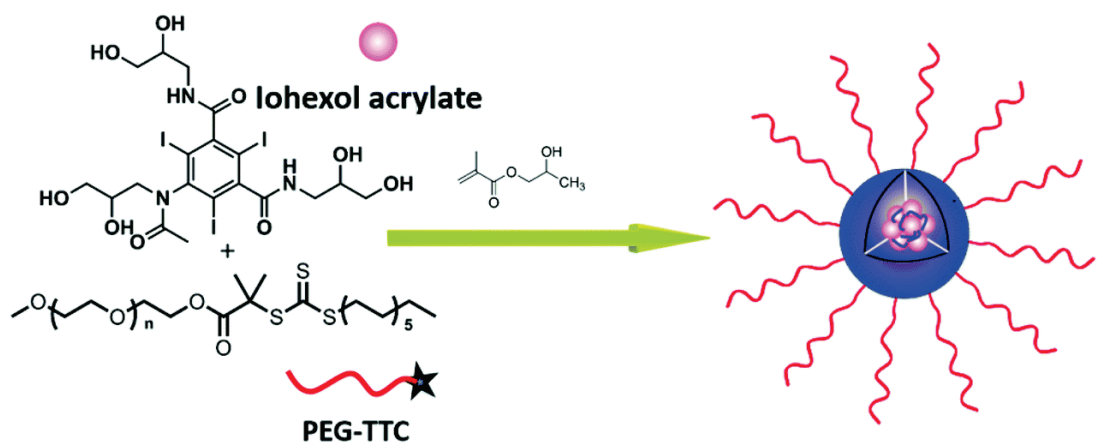

B
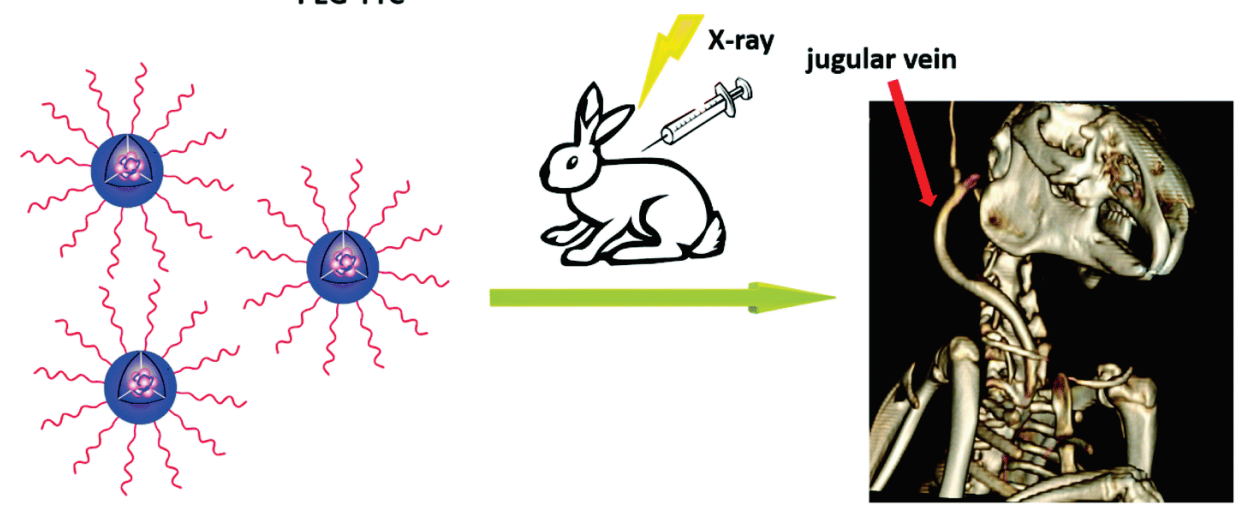

Figure 15. (A) Synthesis of cross-linked iohexol-containing nanoparticles (INPs) by PISA. (B) Application of INPs in X-ray computed tomography. Adapted from ref. ${ }^{72}$.

In addition to linear PEG/PEO derivatives, POEGMA was also intensively investigated as solvophilic block, since not only they share the same 'stealth' properties as linear PEG, ${ }^{75,76}$ but they can also confer thermoresponsive properties, ${ }^{77}$ depending on the OEG sidechain lengths. Like linear PEG-based nanoparticles obtained from PISA, POEGMA- $b$-PHPMA diblock copolymer nanoparticles have also been successfully used to encapsulate various biomacromolecules.

This was illustrated by the encapsulation of Gox during the synthesis of POEGMA- $b$ PHPMA or PGMA- $b$-PHPMA diblock copolymer nano-objects via photo-PISA (405 nm visible light) under oxygen-tolerant conditions (Table 1). ${ }^{69}$ Gox and glucose $(0.1 \mathrm{M})$ were added prior to 
the photoinitiation to ensure the constant removal of oxygen. It was also shown that the presence of Gox did not influence the encapsulation of other biomacromolecules as HRP and BSA were successfully loaded into PGMA40- $b$-PHPMA 250 vesicles with a loading efficiency of about $50 \%$.

The chemical conjugation of proteins, which represents an appealing alternative to their simple physical encapsulation, has also been reported with such systems. For instance, interferon$\alpha$ (IFN) was linked to an ATRP initiator, subsequently used to conduct PISA by consecutive polymerization of OEGMA and HPMA to form the solvophilic and solvophobic blocks, respectively (Figure 16). ${ }^{70}$ Well-controlled and narrowly-dispersed IFN-POEGMA- $b$-PHPMA copolymer micelles with size around $112 \pm 23 \mathrm{~nm}$ in diameter were obtained, despite some disassembly at low concentration $\left(0.5 \mu \mathrm{g} \cdot \mathrm{mL}^{-1}\right)$. Importantly, the PISA process did not alter the secondary structure of IFN as observed by circular dichroism (CD). Their biological evaluation, compared to that of free IFN, showed similar antiproliferative activities, but prolonged circulation time $(83.8 \mathrm{~h}$ for IFN-POEGMA- $b$-PHPMA micelles, $49.5 \mathrm{~h}$ for FDA-approved PEGylated interferon- $\alpha$ PEGASYS, $31.3 \mathrm{~h}$ for IFN-POEGMA and $0.834 \mathrm{~h}$ for free IFN). Eventually, IFNPOEGMA- $b$-PHPMA micelles completely suppressed tumor growth with $100 \%$ animal survival, whereas PEGASYS and IFN were much less effective at the same dose.
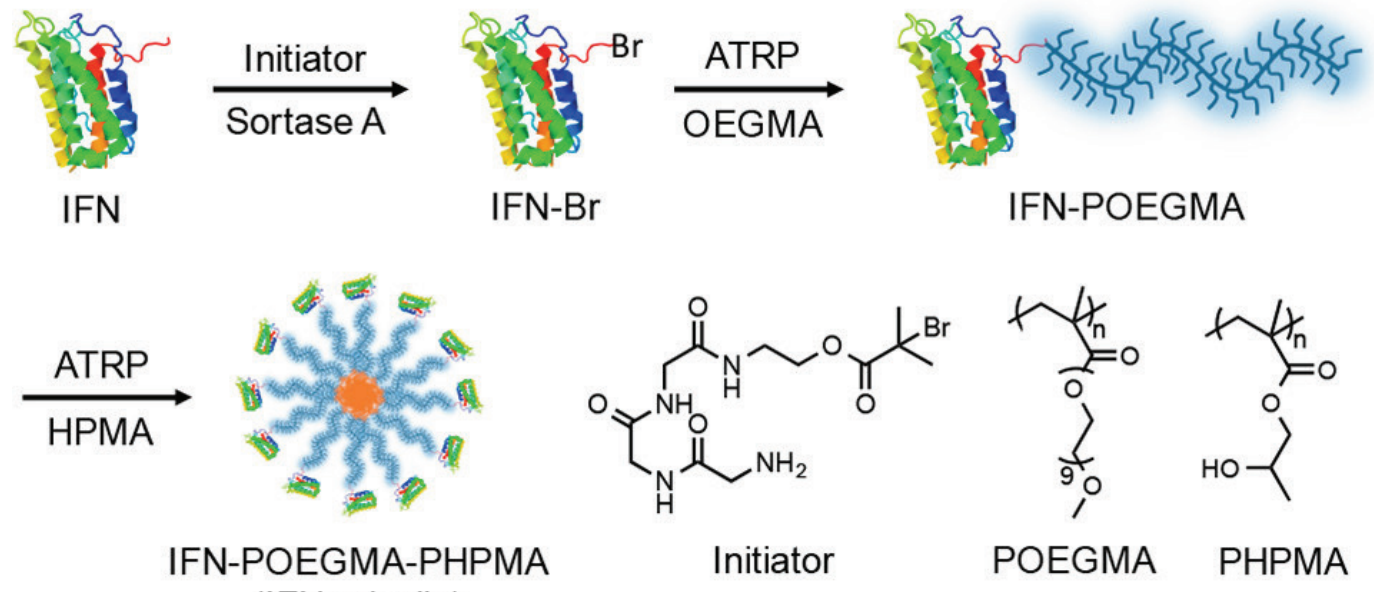

Figure 16. Synthesis of IFN-POEGMA- $b$-PHPMA micelles by polymerization-induced self-assembly. Adapted from ref. ${ }^{70}$. 
Another interesting pathway to link biomacromolecules by PISA is through host-guest interaction. ${ }^{73} \mathrm{~A} \beta$-cyclodextrin $(\beta-\mathrm{CD})$ was linked onto the RAFT agent further engaged in PISA to produce $\beta$-CD-POEGMA $13-b$-PHPMA nanoparticles with different shapes (Figure 17). They were then incubated with $\mathrm{Au}$ nanoparticles previously conjugated to a polymer exhibiting adamantane moieties, used as guest molecules for binding with $\beta$ - $\mathrm{CD}$ moieties. High binding efficiency $\left(\mathrm{K}_{\mathrm{a}} \approx 10^{4} \mathrm{M}^{-1}\right)$ were determined and TEM demonstrated an efficient way to produce polymer-based nanocomposites by PISA.

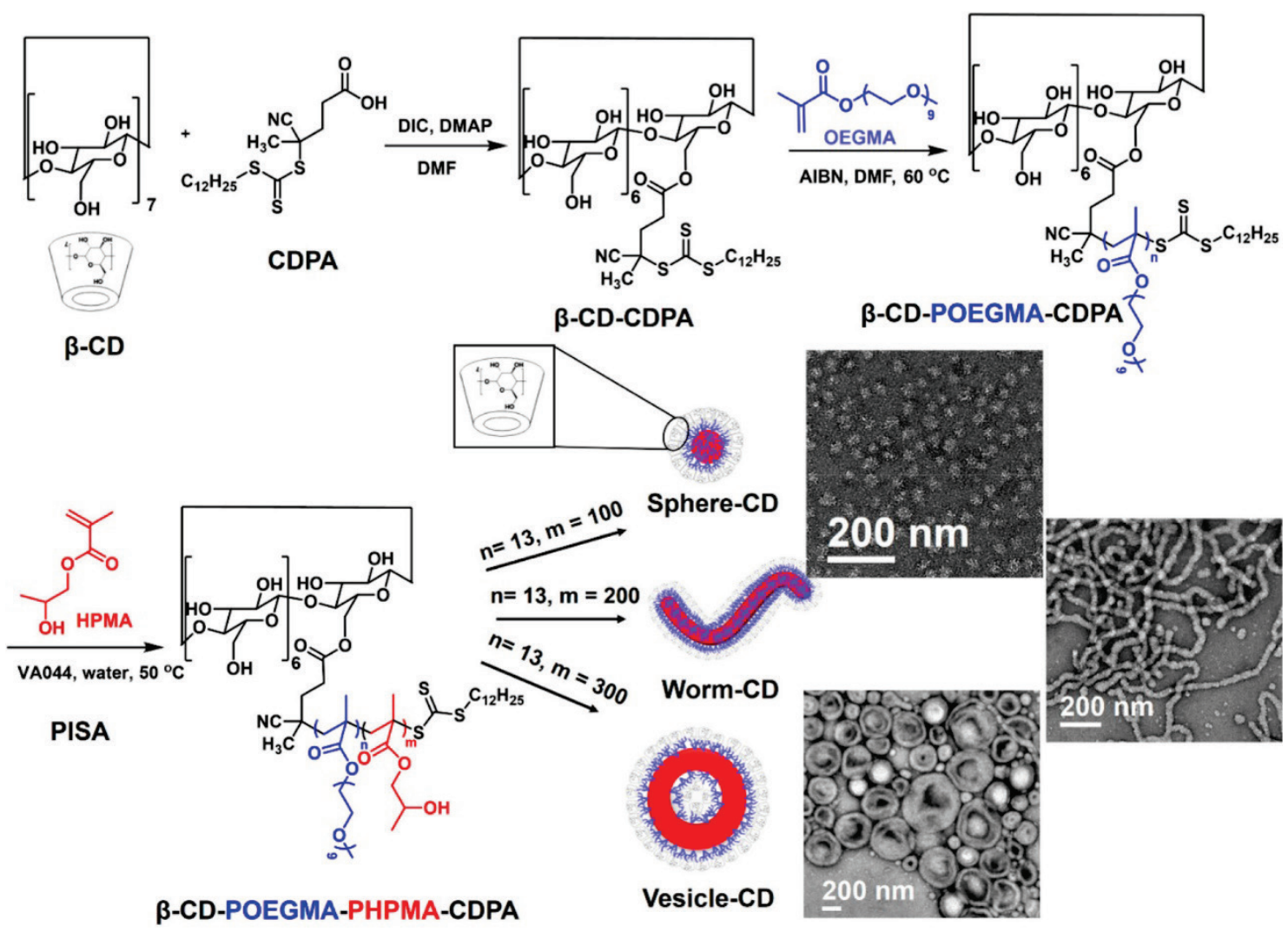

Figure 17. Synthesis of $\beta$-cyclodextrin surface-functionalized poly[oligo(ethylene glycol) methyl ether methacrylate]- $b$-poly(hydroxylpropyl methacrylate) ( $\beta$-CD-POEGMA- $b$-PHPMA) diblock copolymer nano-objects via RAFT aqueous dispersion polymerization, and corresponding TEM images. Adapted from ref. $^{73}$. 
Despite their non-biocompatibility, other polymers have been investigated as the solvophobic block for PISA to generate sophisticated nano-objects for potential applications in drug delivery ${ }^{78-}$ ${ }^{81}$ and imaging. ${ }^{82}$ The molecules of interest were physically encapsulated during polymerization or grafted to the polymer scaffold; either by a post-modification method, or after prior to conjugation to a monomer (also termed "grafting through" approach). ${ }^{83}$

In fact, the first example of in situ encapsulation into PEGylated nano-objects from PISA was reported with polystyrene (PS) as a solvophobic block. POEGMA- $b$-PS nanoparticles were obtained by PISA and Nile red was used as a model molecule to confer fluorescence. ${ }^{79}$ Interestingly, when the chain extension was performed with styrene and vinyl benzaldehyde as a comonomer, this allowed for the nanoparticle crosslinking and the coupling of doxorubicin (Dox) via establishment of a $\mathrm{pH}$-sensitive imine bond, with $5 \mathrm{wt} . \%$ drug loading and $67 \%$ coupling efficiency. ${ }^{80}$ Nano-objects with different morphologies were prepared and their cellular uptake on MCF-7 breast cancer cells showed superior internalization of rod-like nanoparticles than spheres and vesicles. No toxicity up to $10 \mathrm{mg}$. $\mathrm{mL}^{-1}$ was noticed with empty nano-objects but the Doxloaded counterparts exhibited IC50 values in the $0.3-2.2 \mu \mathrm{M}$ range. Influence of size and shape on the in vivo biodistribution of PGMA- $b$-POEGMA- $b$-PS copolymer nano-objects obtained by PISA was studied. ${ }^{84}$ The short surface PGMA block served to link a radiolabelling agent allowing for biodistribution study in nude mice bearing HT1080 tumors. It was found that small spherical nanoparticles exhibited the highest selectivity and tumor accumulation, conversely to rod-like nanoparticles.

Robust protocols have also been developed for the linkage of drugs and imaging molecules to PEGylated nano-objects from PISA by the "grafting through" approach. Grafting a drug onto a polymer scaffold requires a sufficiently stable chemical linkage between the monomer and the drug during polymerization, but sufficiently labile to release the drug under certain physiochemical/biological conditions. For example, camptothecin (CPT), an anticancer drug used against gastrointestinal cancers, was linked to a methacrylate monomer via a disulfide bond and used in PISA for the synthesis of $\mathrm{PEG}_{113}-b-\mathrm{P}\left(\mathrm{MEO}_{2} \mathrm{MA}-c o-\mathrm{CPTM}\right)$ copolymers, that was further 
chain-extended with BzMA and $N, N^{\prime}$-cystaminebismethacrylamide (CBMA) in ethanol/water to yield core-cross-linked polymer prodrug nanoparticles with $\sim 13 \mathrm{wt} \%$ CPT loading. ${ }^{78}$ CPT was released by cleavage from the nanoparticles in the presence of glutathione (GSH) and cytotoxicity experiments on HeLa cells showed the non-cytotoxicity of empty nanoparticles up to $1 \mathrm{mg} \cdot \mathrm{mL}^{-1}$ and the significant cytotoxicity of CPT-loaded nanoparticles from $100 \mu \mathrm{g} \cdot \mathrm{mL}^{-1}$. The polymer prodrug nanoparticles were also significantly internalized by the cells.

Europium (III) (Eu(III)) complexes are widely used as fluorescent lanthanide probes for cell imaging or biosensing applications. ${ }^{82}$ To incorporate such probes into nanoparticles via PISA, a POEGMA macro-CTA was chain extended with Eu(III)-bis(2-thenoyltrifluoroacetone)-monoacrylate-1,10-phenanthroline (Eu(TTA) $2(\mathrm{AA})$ phen) and methyl methacrylate (MMA) in water/acetonitrile $1: 1$ mixture at $80^{\circ} \mathrm{C}$. Spherical and narrowly dispersed POEGMA- $b$-P(MMAco-Eu(TTA)2(AA)phen) (poly(PMEu)) nanoparticles were obtained, with excellent quantum yield $(\sim 37 \%)$, a comparable lifetime of $312.4 \mu$ s and good photostability at acidic and neutral $\mathrm{pH}$. Elemental mapping by scanning transmission electron microscopy revealed that Eu atoms were equally distributed in poly(PMEu) nanoparticle. The cell imaging study showed clear internalization of the nanoparticles with a bright signal in the cytoplasm, revealing great potential as nanoprobes for bioimaging applications.

Conferring stimuli-responsiveness to PISA-derived PEGylated nano-objects is also of high interest. Azobenzene and its derivatives exhibit promising photo-responsive properties ${ }^{85}$ and are sensitive to reducing agents like hydrazine, $\mathrm{Na}_{2} \mathrm{~S}_{2} \mathrm{O}_{4}$ and azoreductase; a feature that can be implemented on polymer nanoparticles. For instance, azobenzene-based polymer nanoparticles for the in situ encapsulation of Dox were investigated (Figure $18 \mathrm{a}) .{ }^{81} \mathrm{~A} \mathrm{PEG}_{4 \mathrm{k}}$ macro-CTA was chainextended under PISA conditions with butyl methacrylate (BMA) and an azobenzene derived methacrylate (TPE-AZO-MMA) in a dioxane/water mixture at $70^{\circ} \mathrm{C}$ to yield nanoparticles with different morphologies. The concomitant nanoparticle dissociation and Dox-release was shown in the presence of $\mathrm{Na}_{2} \mathrm{~S}_{2} \mathrm{O}_{4}$ solution, which was more effective compared with the non-azoreductase Dox-loaded nanoparticles $(58.5 \%$ vs. $<10 \%$ release). Blank nanoparticles exhibited high 
cytocompatibility whereas Dox-loaded nanoparticles pre-activated with azoreductase led to similar cell viability than free Dox (Figure 18b). Crosslinking via establishment of disulfide linkages during the formation of vesicles has also been implemented via the use of disulfide difunctional monomers. ${ }^{74}$ Dual responsive ( $\mathrm{pH}$ and reductant) vesicles with crosslinked membrane and a linear PEG shell were formed, whose reduction in the presence of DL-dithiothreitol under acidic conditions led to disaggregation.

a)
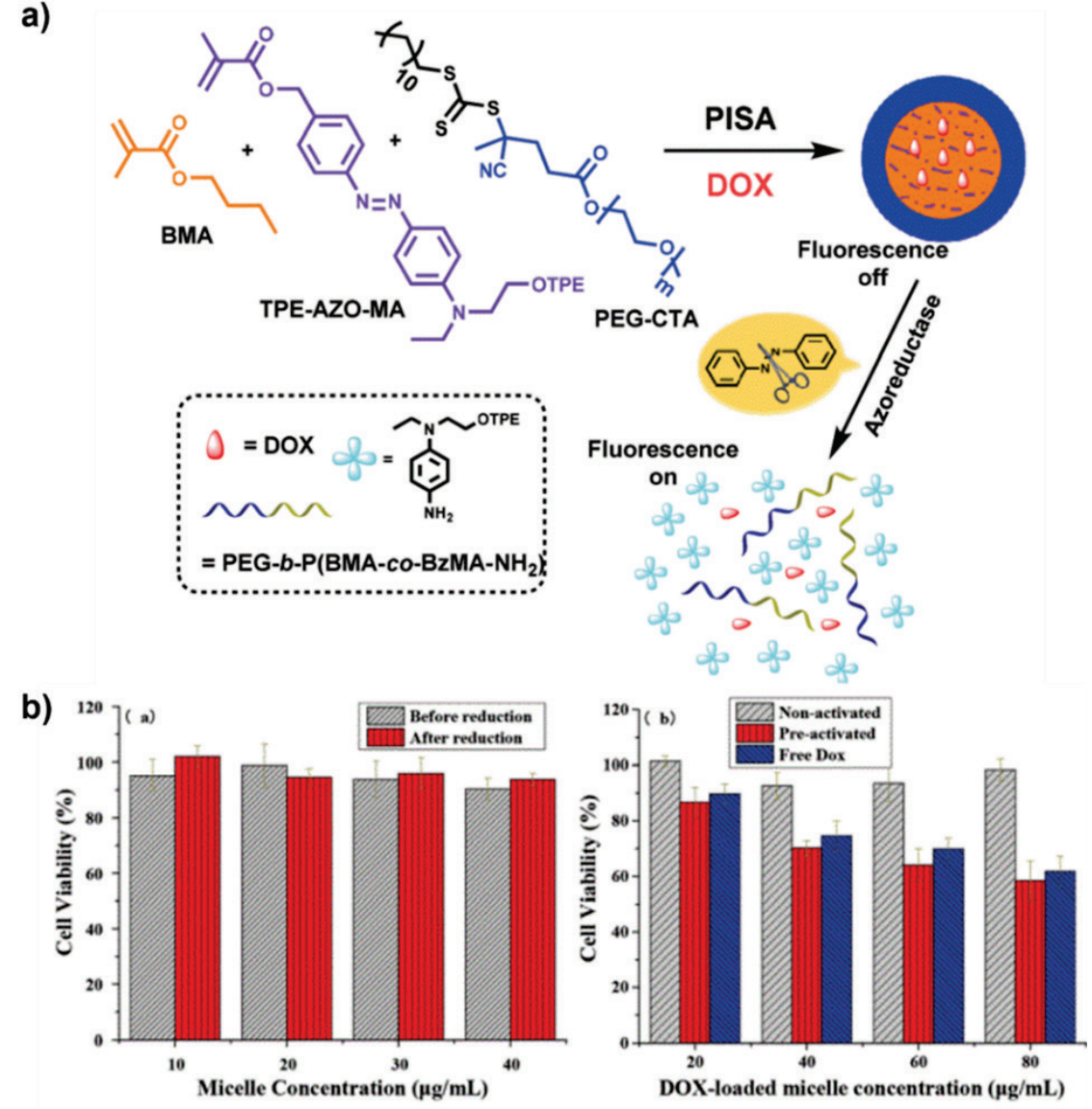

Figure 18. (a) Synthesis of azobenzene-triggered responsive nanoparticles and in-situ encapsulation of Dox by PISA. (b) MTT assays on healthy L929 cells of empty nanoparticles before and after reduction, and on CT26 cancer cells of non-activated or pre-activated Dox-loaded nanoparticles, or free Dox. Adapted from ref. ${ }^{81}$. 
Boron neutron capture therapy (BNCT) is a binary radiotherapeutic modality that relies on the injection of ${ }^{10} \mathrm{~B}$-containing compounds into tumours followed by thermal neutron beam irradiation. This approach has attracted considerable attention in the field of polymer science, ${ }^{86}$ for instance through the design of boron-containing nanoparticles. However, traditional approaches result in low boron contents and tedious synthesis procedures. The first PISA process to formulate boronrich containing nanoparticles was reported by chain extension of a POEGMA macro-CTA with a boronate ester methacrylate monomer (BMMA) in methanol followed by transfer to water (Figure 19). The surface of the nanoparticles was also functionalized by 3 -aminobenzeneboronic acid from a $\alpha$-NHS copolymer to achieve specific targeting of sialylated epitopes on the membrane of solid tumors. Preliminary biological evaluation showed no cytotoxicity on RAW 264.7 and HUVEC cells up to $100 \mu \mathrm{g} . \mathrm{mL}^{-1}$, as well as low cellular accumulation.

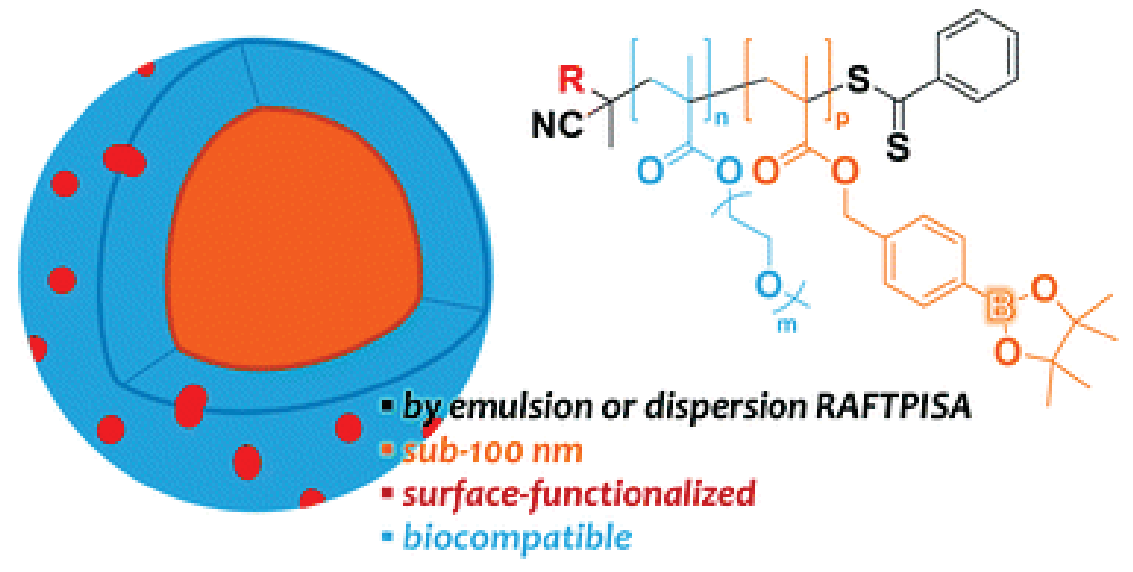

Figure 19. Structure of poly[oligo(ethylene glycol) methacrylate]-b-poly(4-pinacolboronylbenzyl methacrylate) (POEGMA- $b$-PBMMA) copolymer for the synthesis of nanoparticles by emulsion of PISA for application in boron neutron capture therapy (BNCT). Reproduced from ref. ${ }^{87}$.

\subsubsection{Poly(2-hydroxypropyl) methacrylamide}

PHPMAAm has been recognized as a promising hydrophilic polymer used in nanomedicine ${ }^{88}$ as an alternative to PEG, which makes it naturally suitable as a solvophilic block for PISA. This was 
illustrated by the preparation of nano-objects by polymerization-induced self-assembly and reorganization (PISR). It relied on the photoinduced cross-linking of nanoparticles with a PHPMAAm outer shell and their further use as drug carriers. ${ }^{89,90}$ The chain extension from a PHPMAAm macro-CTA was conducted with 7-(2-methacryloyloxy-ethoxy)-4-methyl-coumarin (CMA) and either photosensitive 2-nitrobenzyl methacrylate (NBMA), ${ }^{89}$ or (2(diisopropylamino)ethyl methacrylate (DIPEMA) ${ }^{90}$ to highlight distinct potential functions. In both examples, nano-objects with different morphologies were observed and their UV irradiation led to crosslinked nano-objects via dimerization of the coumarin moieties. Photoinduced cleavage of the NBMA units produced PHPMAAm- $b-\mathrm{P}(\mathrm{CMA}-c o-\mathrm{NBMA})$ copolymer nanoparticles with core carboxylic acid groups that were conjugated to Dox up to high drug loadings ( $25 \mathrm{wt} . \%)$, $\mathrm{pH}-$ sensitive release and efficient endosomal escape. For PHPMAAm- $b$-P(CMA-co-DIPEMA) copolymer nanoparticles, fine tuning the UV irradiation served to adjust the pH-regulated membrane permeability through protonation of the tertiary amine groups of DIPEMA.

A mixture of PHPMAAm and PDEAEMA macro-CTAs was used to produce $\mathrm{pH}-$ responsive polymer prodrugs nanoparticles by PISA after their chain extension with a camptothecin analogue methacrylate (CPTM) monomer in ethanol (Figure 20). ${ }^{91}$ Interestingly, the cellular uptake by HeLa cells showed higher internalization of the prodrug nanoparticles at $\mathrm{pH}=$ 6.8 than $\mathrm{pH}=7.4$, suggesting that protonation enhanced the cellular uptake mechanism. Such observation was corroborated by similar experiments performed with nanoparticles exhibiting a pure PHPMAAm shell. 


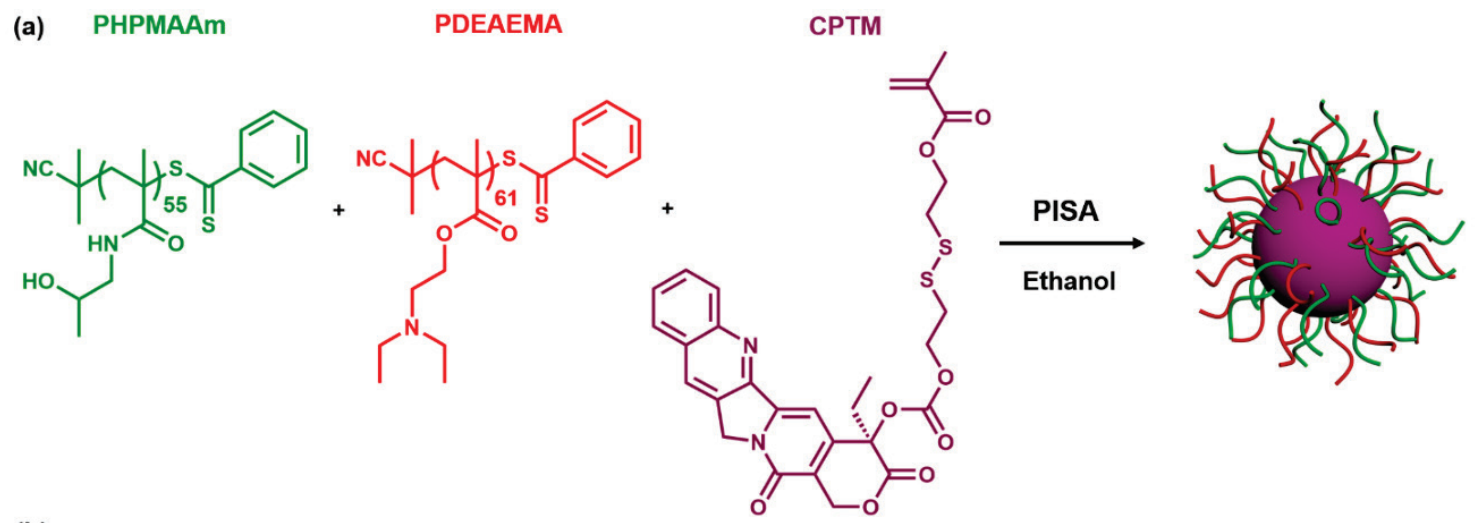

(b)

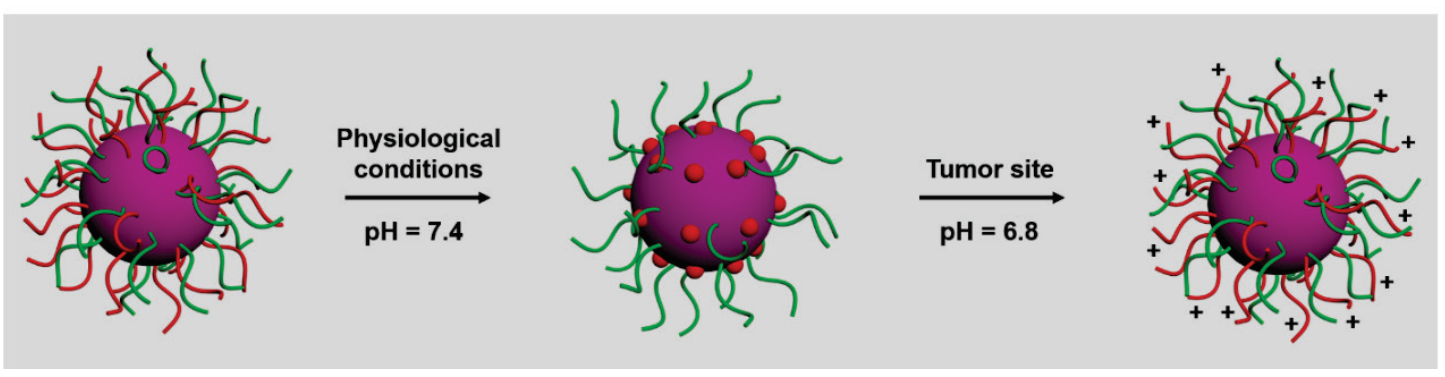

Figure 20. (A) Polymerization-induced self-assembly using CPTM as the monomer and a mixture of PHPMAAm and PDEAEMA macro-CTAs in ethanol to prepare PHPMAAm/PDEAEMA-stabilized polymer prodrug nanoparticles. (B) $\mathrm{pH}$-responsive charge-reversal of the prodrug nanoparticles. Adapted from ref. ${ }^{91}$.

Polyion complexes (PICs) have an interesting potential for biomedical applications. ${ }^{92}$ Similarly to other vinyl polymer nanoparticles, traditional formulation methods limit their scale-up production and prevent high solids content nanoparticles to be obtained. PIC-PISA relied on chain-extension of a PHPMAAm macro-CTA by a $\mathrm{NH}_{3}{ }^{+}$-monomer (2-aminoethylacrylamide hydrochloride, AEAM) in presence of poly(sodium 2-acrylamido-2-methylpropanesulfonate) (PAMPS) as a PICtemplate to yield nano-objects from spheres to network. ${ }^{92}$ The concept of polymerization-induced electrostatic self-assembly (PIESA) was also reported. ${ }^{93}$ This process can give easy access to low dimensional PICs nano-objects (e.g., nanospheres, nanowires, large-area nanofilms, porous nanofilms, vesicles and multi-compartmental vesicles) that could be of high interest for biomedical applications given its versatility in terms of hydrophilic polymers ${ }^{94-96}$ and preparation methods. ${ }^{97-}$ 101 


\subsubsection{Poly(glycerol methacrylate)}

Since poly(glycerols) are considered as an alternative to PEG, ${ }^{12}$ PGMA has been considered as a solvophilic polymer in PISA. Biocompatible PGMA- $b$-PHPMA copolymer nano-objects, with various morphologies can be prepared by PISA, in the same way as the PEG- $b$-PHPMA counterparts (Figure 21a,b). ${ }^{69,102,103}$ Interestingly, a thermo-reversible hydrogel can be formed at room temperature at $10 \mathrm{wt} \%$ solids content by interworm entanglement. ${ }^{104}$ Cytotoxicity assays revealed the non-toxicity of formed nanoparticles on mammalian cells (H357 keratinocytes) at concentration up to $8 \mathrm{wt} . \%$. To illustrate potential applications, silica (Figure 21c) and BSA were successfully encapsulated into the core or the lumen of the nanoparticles, respectively. ${ }^{105}$ Such a PGMA-PHPMA diblock copolymer worm gel has also been shown to be an excellent synthetic mimic for some types of natural mammalian mucins and also be a suitable bioinert 3D matrix for human pluripotent stem cells. ${ }^{106}$ Their ice recrystallization inhibition properties have also been demonstrated on red blood cells, ${ }^{107}$ making them a suitable alternative to the current state-of-theart strategies for cryopreservation, as they do not require organic solvents (e.g., glycerol, DMSO) and they do not have the limitation of ice-recrystallization inhibitors (e.g., antifreeze (glycol)proteins (AF(G)Ps)), which are challenging to synthesize and have biocompatibility issues. 
a)

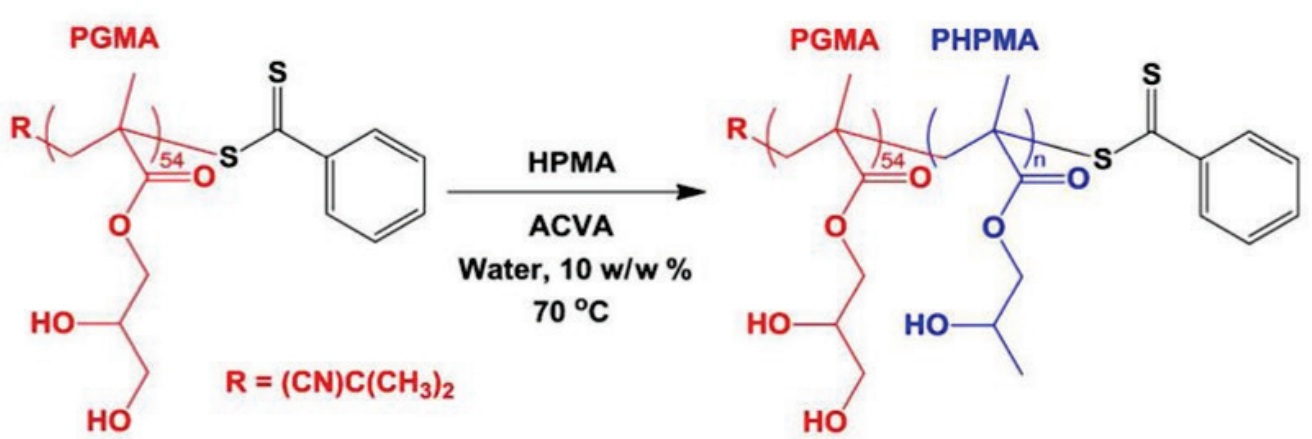

b)
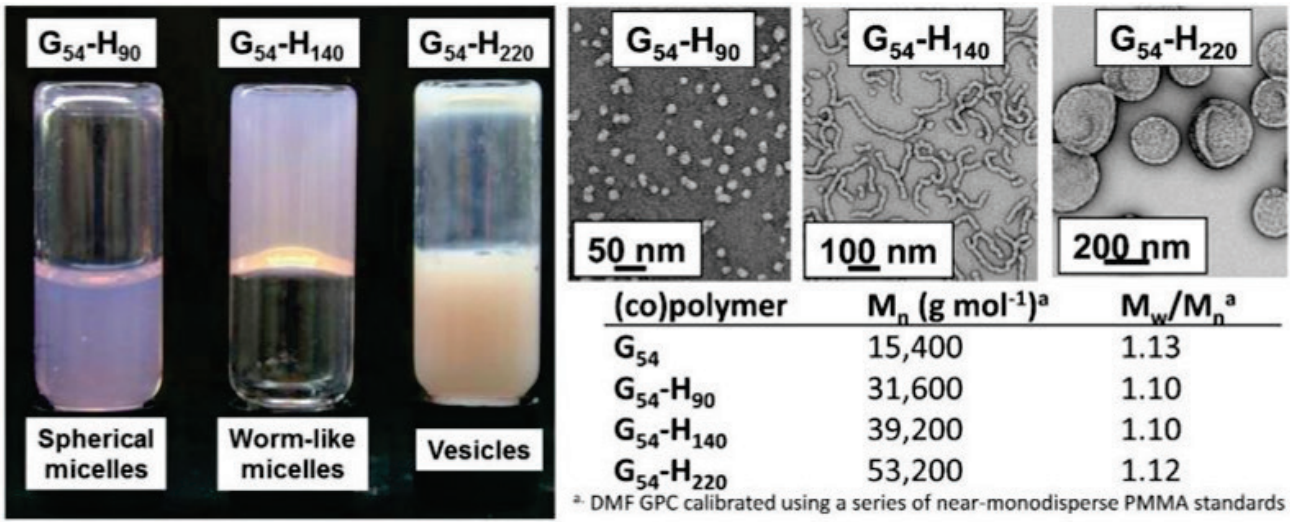

(co)polymer

$\mathrm{G}_{54}$

$\mathrm{G}_{54}-\mathrm{H}_{90}$

$\mathbf{M}_{\mathrm{n}}\left(\mathrm{g} \mathrm{mol}^{-1}\right)^{\mathrm{a}}$

$\mathrm{M}_{\mathrm{w}} / \mathrm{M}_{\mathrm{n}}^{\mathrm{a}}$

$\mathrm{G}_{54}-\mathrm{H}_{140}$

15,400

1.13

$\mathrm{G}_{54}-\mathrm{H}_{220}$

31,600

1.10

39,200

1.10

2. DMF GPC calibrated using a series of near-monodisperse PMMA standards

c)
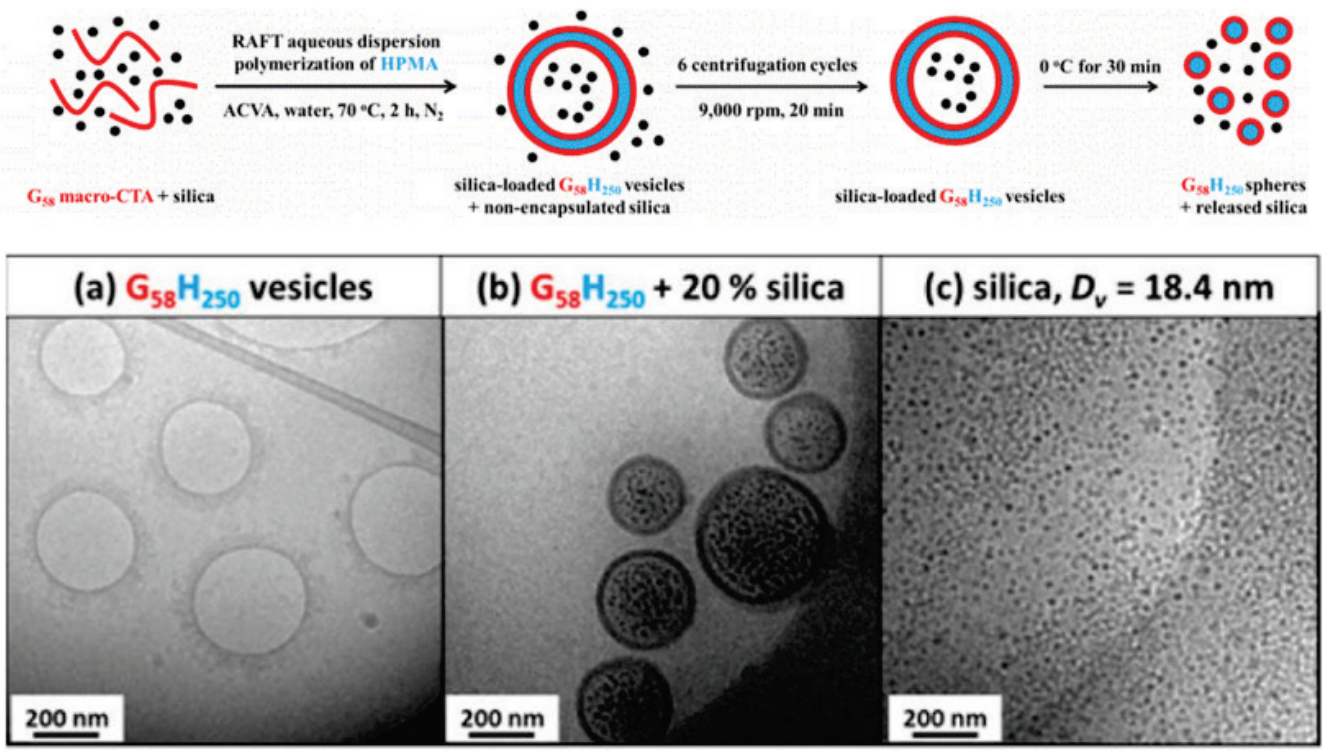

Figure 21. (a) Synthesis of PGMA- $b$-PHPMA diblock copolymer by PISA. (b) Representative samples and their TEM images showing nanoparticles with different morphologies. (c) In-situ encapsulation of silica particles during PISA and cyro-TEM images of: (a) empty vesicles, (b) silica-loaded vesicles and (c) silica alone. Adapted from ref. ${ }^{104}$ and ${ }^{105}$. 
Other studies reported similar PGMA- $b$-PHPMA diblock copolymers nano-objects by PISA but with modification of their solvophilic shell. For instance, by polymerizing HPMA under aqueous PISA conditions from a mixture of PGMA and a poly(galactose methacrylate) (GalSMA) macroCTAs, galactosylated diblock copolymer nano-objects with tunable morphologies (nanospheres, worm-like micelles and vesicles) were formed (Figure 22). ${ }^{108}$ They showed specific binding to a galactose-specific lectin (RCA120) where it was shown that worms and vesicles gave greater interactions than spheres. The nano-objects were not toxic to Human dermal fibroblasts (HDF) up to $1 \mathrm{mg} \cdot \mathrm{mL}^{-1}$. They also exhibited significant internalization into cells followed by release of their payload, which may open new pathways in drug delivery.

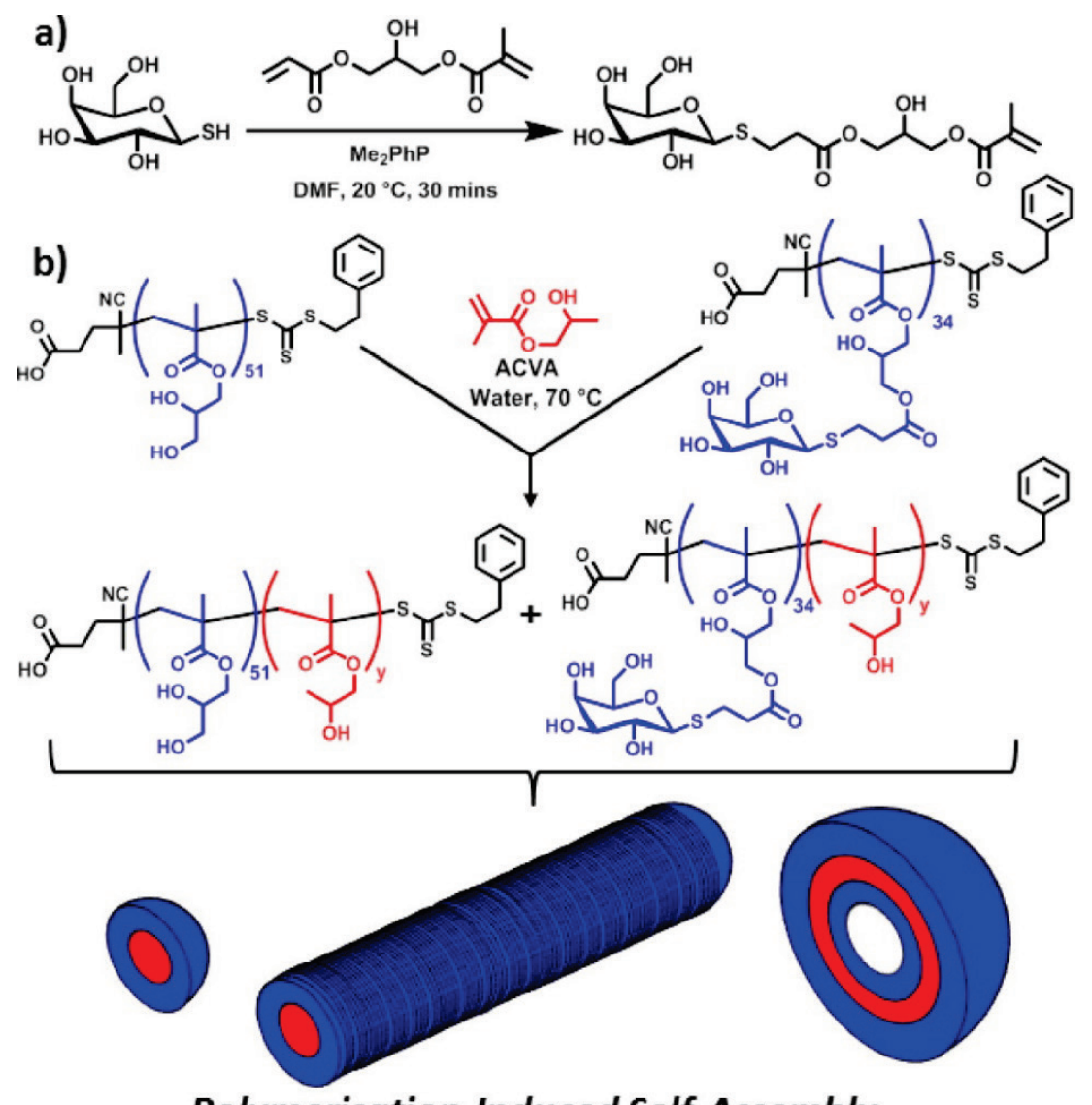

Polymerisation-Induced Self-Assembly

Figure 22. (a) Synthesis of a GalSMA monomer. (b) Synthesis of diblock copolymer nano-objects (spheres, worms or vesicles) via PISA of HPMA using a binary mixture of RAFT macro-CTAs based on PGMA and PGalSMA. Adapted from ref. ${ }^{108}$. 
Introducing 3 mol.\% of 2-methacryloyloxyethyl phosphorylcholine (MPC), to promote selective intracellular uptake, into a PGMA macro-CTA followed by chain extension with (diisopropylamino)ethyl methacrylate (DPA) by PISA was studied to produce nano-objects with a framboidal morphology as a synthetic mimicry of the Dengue virus to target triple-negative (TN) breast cancer cells. ${ }^{109}$ Encapsulation of plasmid DNA into framboidal vesicles was performed by electroporation followed by its successful release in the endolysosomal compartments.

Another type of modification of the solvophilic PGMA block was achieved by copolymerization of a self-assembling tripeptide-based methacrylamide monomer (Mam-GFF,

Mam-Gly-Phe-Phe- $\mathrm{NH}_{2}$ ) with GMA. ${ }^{110}$ The subsequent aqueous PISA process with HPMA produced nano-objects exhibiting peptide moieties on their surface and an unique thermoreversible transformation from fiber-to-worm or rod-to-sphere shape, depending on the temperature. Such a reversible morphology change phenomenon was explained by the local rearrangement of the peptide moieties on the surface. Alternatively, Mam-GFF and another tripeptide-based methacrylamide monomer Mam-FGD (Mam-Phe-Gly-Asp- $\mathrm{NH}_{2}$ ) were also used as core-forming materials with PGMA as the solvophilic block. ${ }^{111}$ It resulted in various fibrous structures (e.g., fiber-, flake- and leaf-like) or spherical vesicles, depending on the copolymer composition and solvent used. This highlighted the crucial influence of the self-assembling peptides moieties on the final morphologies.

\subsubsection{Poly[2-(methacryloyloxy) ethyl phosphorylcholine]}

Zwitterionic polymers are known for their biocompatibility and antifouling properties, as well as their ability to reduce or avoid non-specific interactions with proteins. ${ }^{58} \mathrm{PMPC}$ is one of the most studied zwitterionic polymers as it contains a phosphorycholine group that mimics the phospholipid bilayer of cell membranes. Early studies of alcoholic or aqueous PISA have witnessed the successful use of PMPC as the solvophilic block ${ }^{12,113}$ and the potential of the resulting PMPC-coated nanoparticles in nanomedicine. ${ }^{114,115}$ For instance, the drug loading can 
significantly affect the morphology of the nanoparticles during PISA and therefore their biological performances. ${ }^{115}$ This was illustrated by addition of curcumin as a model drug during the synthesis of PMPC- $b$-PMMA nano-objects by PISA, resulting in a shift of the phase diagram toward different morphologies. Curcumin was mainly located in the core of micelles and worms, whereas it was found in the shell of vesicles.

In another example, MPC was copolymerized with (4-( $N$-( $S$-penicillaminylacetyl)-amino) phenylarsonous acid) methacrylate monomer (PENAO) to yield organo-arsenical therapeutic drug-containing solvophilic macro-CTA that was chain extended by MMA under PISA conditions (Figure 23). ${ }^{114}$ It resulted in polymer prodrug micelles of $30-40 \mathrm{~nm}$ in diameter that exhibited tunable cytotoxicity as function of the PMPC chain length: reducing the PMPC chain length from 37 to 13 repeating units led to a 5-fold increase in cytotoxicity. These nanoparticles can be postfunctionalized on their surface by triphenylphosphonium (TPP) as a mitochondria targeting agent, resulting in an improvement of tumor penetration and cytotoxicity. ${ }^{116}$ Interestingly, when OEGMA was used instead of MPC, the surface functionalization of resulting nanoparticles by TPP had little effect on their biological performances, likely because of a higher steric hindrance around TPP moieties caused by OEGMA side chains compared to MPC moieties. 


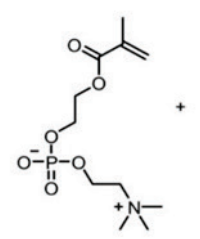

MPC

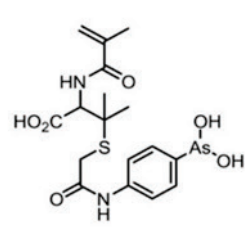

PENAO

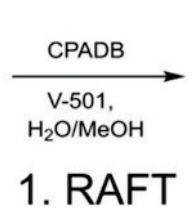

1. RAFT
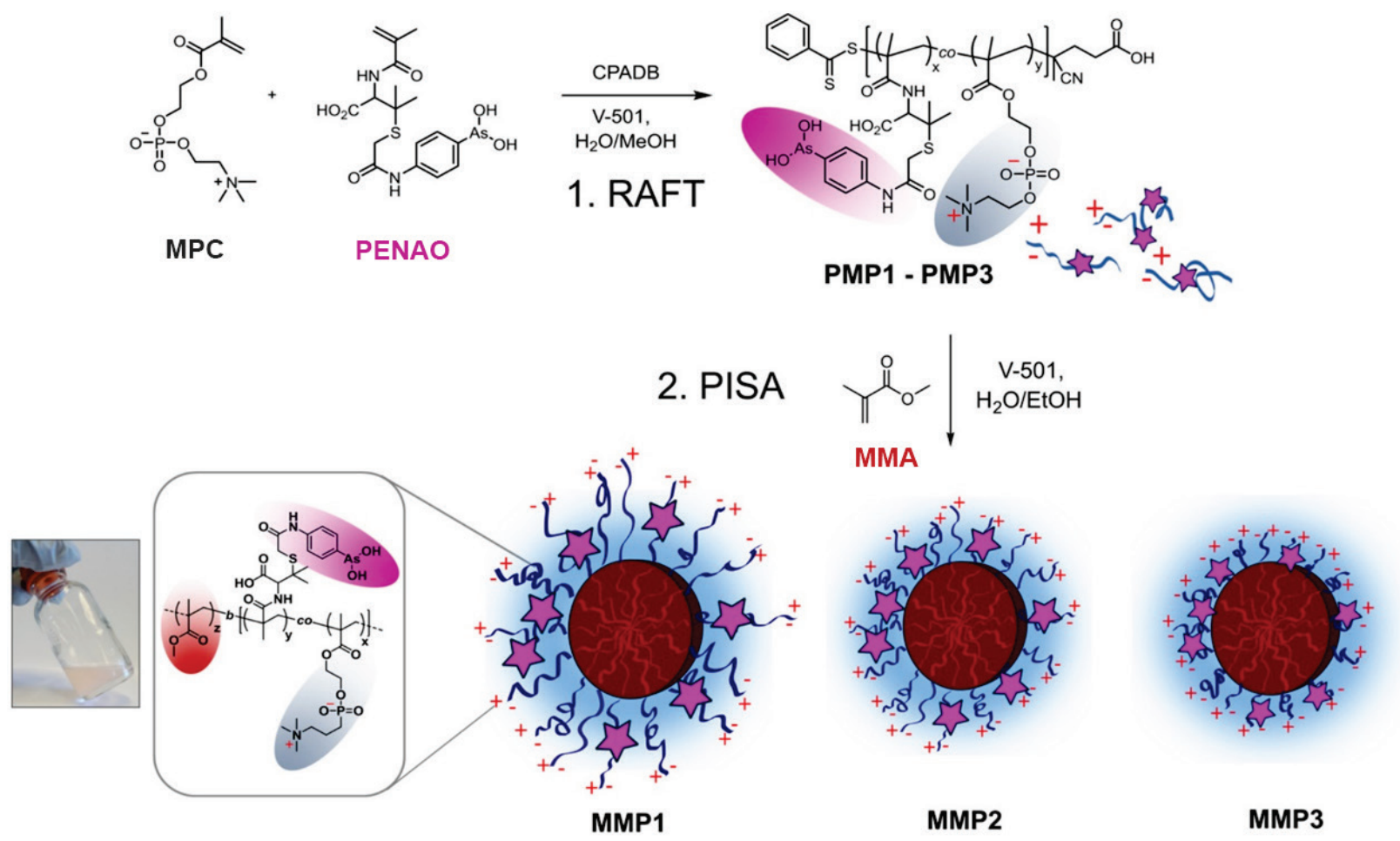

Figure 23. Synthesis of P(MPC-co-PENAO) copolymers followed by the formation of core-shell P(MPC$c o$-PENAO)- $b$-PMMA nanoparticles. Adapted from ref. ${ }^{114}$.

\subsubsection{Poly(acrylamides) and their derivatives}

Poly(acrylamide) (PAAm) has been reported to be non-immunogenic, highly resistant to protein and cytocompatible although its monomer is listed as possibly carcinogenic to humans. ${ }^{12}$ Early examples of PAAm-based nanoparticles produced by emulsion or dispersion polymerization have shown its biocompatibility in vitro and during ex vivo experiments, ${ }^{117}$ which prompted study of its use as solvophilic polymer for PISA. For examples, a thio-ether conjugated acrylamide monomer was designed to produce oxidative responsive micelles by PISA. ${ }^{118} \operatorname{Poly}(N-$ acryloylmorpholine)- $b$-( $N$-acryloylthiomorpholine) (PNAM- $b$-PNAT) diblock copolymer nanoparticles were obtained in a water/dioxane mixture (Figure 24a). The hydrophobic thioether moiety can be transformed into a hydrophilic sulfoxide counterpart, resulting in disassembly of the nanoparticles. Successful internalization in HEK cells was shown, which indicates potential 
application in oxidation-triggered burst release of drugs with tunable time delay (Figure 24b), which was further illustrated by encapsulation of calcein and Gox. ${ }^{119}$

a)
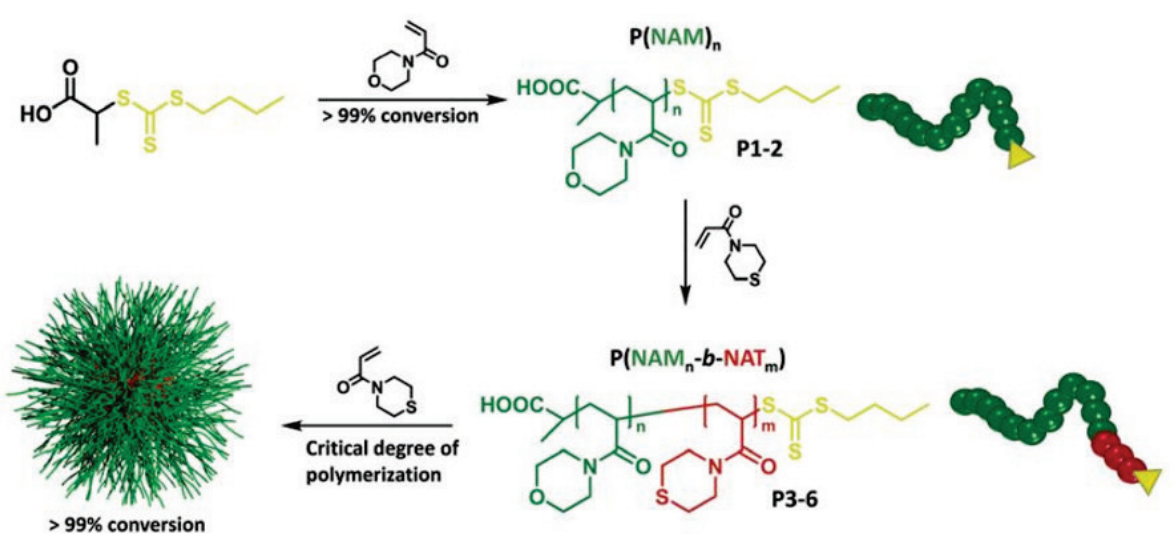

b)
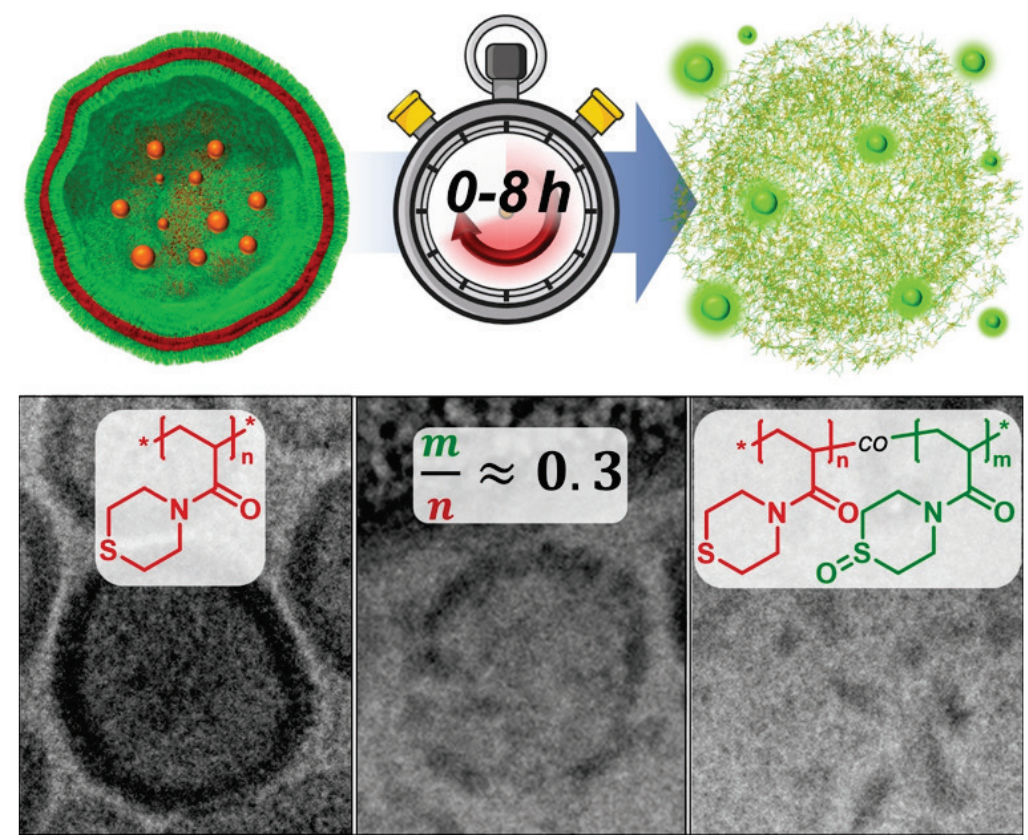

Figure 24. (a) Synthesis of PNAM- $b$-PNAT diblock copolymer nanoparticles by PISA. (b) Application of oxidation-responsive PNAM- $b$-PNAT vesicles in tunable time delay in the burst release. Adapted from ref. 118 and ${ }^{119}$. 
Poly $(N, N$-dimethylacrylamide) (PDMA) was another polyacrylate that is often studied despite its less hydrophilic character than PAAm. Nanoparticles with a PDMA shell have shown interesting applications as ice-recrystallization inhibitors ${ }^{120}$ or for the synthesis of surface-functionalized materials. ${ }^{121-123}$ For example, copolymerization of DMA and an acrylamide monomer functionalized with the proapoptotic peptide KLA (KLAKLAKLAKLAK) was reported as a simple route toward peptide-based nanoparticles by PISA (Figure 25). ${ }^{123}$ Chain extension by photo-PISA of the P(DMA-co-KLAAm) macro-CTA with a mixture of DMA/DAAm in buffer solution $(\mathrm{pH}=5)$ gave stable spherical micelles with tunable size (36-105 $\mathrm{nm}$ in diameter) and high peptide loadings (up to 48 wt.\%). The enhanced proteolytic resistance of the peptidefunctionalized nanoparticles was shown in comparison with the free KLA peptide in the presence of trypsin. Moreover, the nanoparticles significantly enhanced cell uptake permeability by multivalent display of KLA peptides and led to improved apoptosis efficiency in HeLa cells.

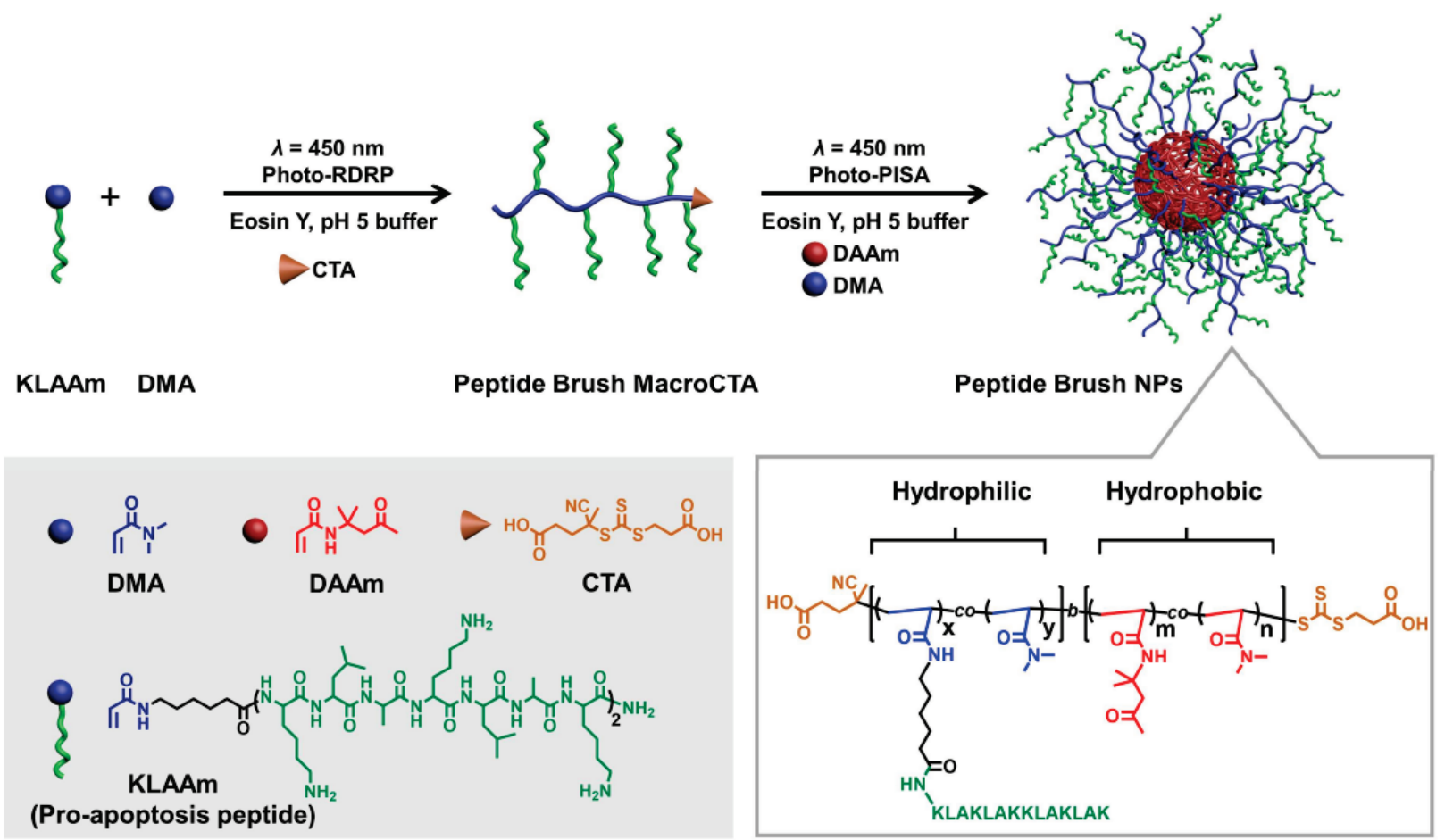

Figure 25. Schematic illustration of the one-pot photo-PISA approach to proapoptotic peptide brush polymer nanoparticles. Adapted from ref. ${ }^{123}$. 
PVP has been recognized as a well-known biocompatible polymer and is currently used in clinical trials. ${ }^{9}$ It was shown that PISA-derived nanoparticles having linear PEG, PDMA or PVP coronas were able to inhibit ice recrystallization, while these polymers had no apparent activity alone. ${ }^{120}$ More interestedly, PVP exhibited ice crystal shaping activity, which opened the way to icecontrolling nano-objects by PISA.

Other supposedly biocompatible polymers have also been investigated as solvophilic blocks for PISA. For example, poly((N-[3-(dimethylamino) propyl] methacrylamide) (PDMAPMA) and PDMAEMA cationic polymers are widely used in gene therapy despite their non-degradability and potential cytotoxicity. ${ }^{124,125}$ A detailed study towards the influence of morphologies was conducted with Dox-conjugated PDMAEMA-b-PMAEBA nanoparticles obtained by PISA. ${ }^{126}$ It was found that polymer prodrug vesicles gave the fastest internalization by HeLa cells, and thus the lowest IC50 values compared with other morphologies. Empty PDMAPMA- $b$-PMMA nanoparticles also demonstrated their non-toxicity on RAW264.7 and MCF-7 cells up to $1 \mathrm{mg} \mathrm{mL}{ }^{-1}$, whereas free Dox and Dox-loaded nanoparticles were much more cytotoxic. ${ }^{127}$

Finely controlling of the surface chemistry and colloidal characteristics of nanoparticles is of utmost importance because these parameters determine the interactions of the nanoparticles with the biological environment. In this context, fluorescent PISA-derived diblock copolymer nanoparticles comprising a solvophilic block with $N$-hydroxyethyl acrylamide (HEAAm) and (ethylene glycol) acrylate (EGA) units, and a PS solvophobic block served as a tool to perform an in-depth investigation on the influence of size, surface chemistry and dynamic flow on their association with endothelial cells (HUVEC) ${ }^{128}$ Carboxylic acid (COOH), tertiary amine (TA), methyl ester and $\mathrm{PEG}_{12}$ were selected as the surface functional groups. Among the different patterns, it was found that increasing the diameter of $\mathrm{COOH}-$ coated nanoparticles gave higher cellular association in flow conditions but lower under static conditions. With TA-coated nanoparticles, larger nanoparticles led to higher cellular associations in both cases. These results provided a better understanding of how different coatings can strongly affect interactions with 
cells under static or flowing conditions, giving insight into how nanoparticles would function in the real human vascular network.

\subsubsection{Other polymers}

Heparin-mimicking nanoparticles were also developed by aqueous PISA using poly(2-acrylamido2-methyl propane sulfonic acid) (PAMPS) as the solvophilic block (Figure 28). ${ }^{129}$ Heparin is a sulfated polysaccharide that plays a significant role in wound healing and tissue engineering, through stabilization of fibroblast growth factors (FGF). The direct one-pot synthesis of stable PAMPS- $b$-PS nanoparticles was reported without need of further purification. Nanoparticles exhibited high cytocompatibility on NIH3T3 cells up to $1 \mathrm{mg} \cdot \mathrm{mL}^{-1}$, but rather significant haemolytic activity compared to natural heparin and PAMPS. Nonetheless, the heparin mimicking nanoparticles gave better results than both heparin and linear PAMPS in terms of cellular proliferation assays, possibly due to the high surface density of sulfonated moieties at the surface of the nanoparticles.

(a)

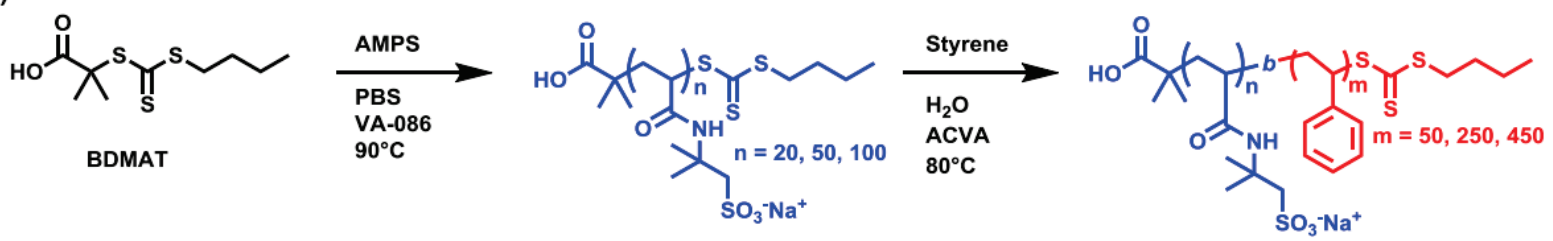

(b)

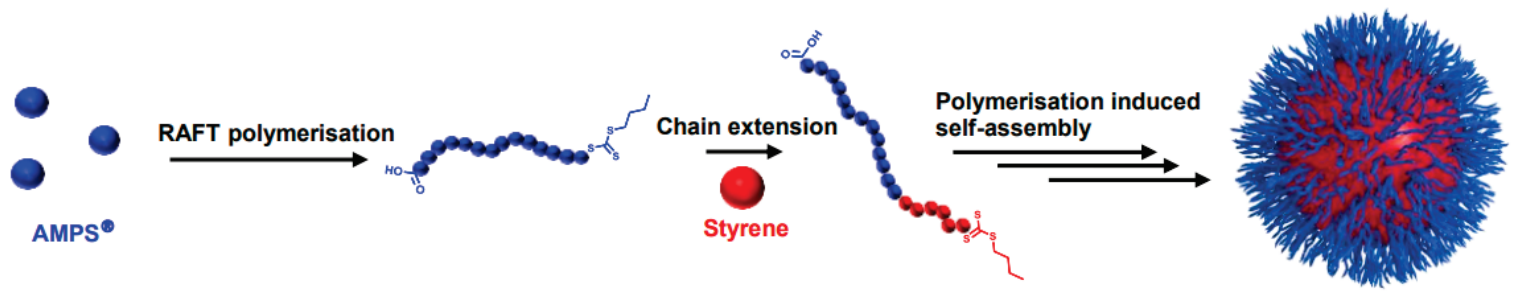

Figure 28. (a) Synthesis scheme and (b) schematic representation for the preparation of poly(2-acrylamido2-methyl propane sulfonic acid) (PAMPS) macro-CTA, and subsequent PISA via chain extension with styrene to generate PAMPS-coated nanoparticles. Adapted from ref ${ }^{129}$. 
Poly(2-oxazoline) (POx) and its derivatives have recently emerged as key biocompatible polymers in the biomedical field. ${ }^{130}$ POx-based nanomaterials synthesised by PISA have recently been investigated. ${ }^{131}$ Poly(2-ethyl-2-oxazoline)-b-PHPMA copolymer nanoparticles with different morphologies were formulated by photoinitiated PISA. The biocompatibility, prolonged circulation in the bloodstream and thermoresponsive properties of the nanoparticles were fully illustrated.

Quite recently, ring-opening metathesis polymerization-induced self-assembly (ROMPISA) $)^{132}$ has emerged as a powerful synthetic tool as alternative to the current PISA methodologies. Several ROMPISA systems with potential in nanomedicines have recently been reported. For instance, ROMPISA can be conducted in acidic phosphate buffer $(\mathrm{pH}=2)$ solutions under air with a PEG-based miscible norbornene monomer leading to diblock copolymer nanoobjects up to $20 \mathrm{wt} . \%$ solids content with different morphologies. ${ }^{133}$ This strategy was then extended to the synthesis of high nitroxide-content nanoparticles relevant for antioxidant delivery. ${ }^{134}$ The ROMPISA process has also been extended to the one-pot aqueous phase synthesis of cisplatin-loaded micelles by the 'grafting through' approach. ${ }^{135}$ An oligo(ethylene glycol) linked norbornene polymer was chain extended with a mixture of a cisplatin norbornene analogue dicarboximide derivative and quaternary amine phenyl norbornene dicarboximide in water at 5 wt.\%, resulting in stable micelles with size ranging from $13 \mathrm{~nm}$ to $81 \mathrm{~nm}$. Scanning transmission electron microscopy energy-dispersive $\mathrm{X}$-ray spectroscopy was used to demonstrate the presence of platinum drugs in the nanoparticles. The cytocompatibility of the nanoparticles was assessed on human cervical cancer (HeLa) and ovarian cancer (CAOV3) cell lines, and MTT assays revealed clear toxicity.

\subsubsection{Biomacromolecules}

Beyond traditional synthetic polymers, natural biomacromolecules such as proteins, sugars, enzymes or DNAs are potential candidates for the fabrication of the shell of PISA nano- 
objects. ${ }^{136,137}$ This section will focus on the biocompatibility of the resulting nanoparticles and their behavior in vitro (Table 2).

Table 2. Biomacromolecules used as the Solvophilic Block in PISA.

\begin{tabular}{|c|c|c|c|}
\hline $\begin{array}{c}\text { Type of } \\
\text { biomacromolecules }\end{array}$ & $\begin{array}{c}\text { Biomacromolecules } \\
\text { used }\end{array}$ & $\begin{array}{c}\text { Anchoring } \\
\text { moiety }\end{array}$ & Ref \\
\hline \multirow{3}{*}{ Proteins } & BSA & RAFT macro-CTA & 138 \\
\hline & HSA & ATRP initiator & 139 \\
\hline & sfGFP & ATRP initiator & 140 \\
\hline \multirow{4}{*}{$\begin{array}{c}\text { Functional } \\
\text { nucleic acids }\end{array}$} & GOx/HRP & ATRP initiator & 141 \\
\hline & $\mathrm{Sgc} 8 / \mathrm{CpG}$ & RAFT macro-CTA & 142 \\
\hline & 19-mer ssDNA & RAFT macro-CTA & 143 \\
\hline & Xyloglucan & RAFT macro-CTA & 144 \\
\hline \multirow[t]{3}{*}{ Polysaccharides } & Alginate & LRP initiator & 145 \\
\hline & Alginate & RAFT macro-CTA & 146 \\
\hline & Dex-g5 & RAFT macro-CTA & 147,148 \\
\hline
\end{tabular}

Protein-initiated PISA offers some advantages such as tunable protein loadings and high loading efficiency (ideally 100\%). Compared to traditional encapsulation of proteins or protein-polymer conjugates, protein-conjugated nanoparticles formed by PISA can therefore exhibit superior stability and protein bioactivity without sophisticated and/or often partially efficient formulation processes. Their presentation on the surface of nanoparticles was also considered beneficial for the crossing of biological barriers and intracellular delivery. ${ }^{149}$ So far, human serum albumin (HSA), mutant superfolder GFP (sfGFP) proteins and BSA have been used as macro initiator for the copolymerization with HPMA by PISA. ${ }^{138-140}$ HSA- $b$-PHPMA nanoparticles were constructed by conjugation of HSA to an ATRP initiator through maleimide/thiol ligation followed by 
polymerization of HPMA via PISA (Figure 26a). ${ }^{139}$ The copolymer growth was confirmed by SDS-PAGE and SEC measurement, and the sphere-to-vesicle transition was confirmed by TEM. No loss of protein activity was found in all cases and no toxicity was observed on cancer cells (MCF-7) and healthy cells (MCF-10A) up to $10 \mathrm{mg} \cdot \mathrm{mL}^{-1}$. Furthermore, attempts to encapsulate GFP led to $\sim 5$ wt.\% loading (Figure 26b). Cellular uptake experiments demonstrated internalization has both HSA- $b$-PHPMA and P-loaded HSA- $b$-PHPMA nanoparticles with a better uptake efficiency compared with free GFP, suggesting that such protein-based nanocarriers can enhance the intracellular delivery of proteins.

a
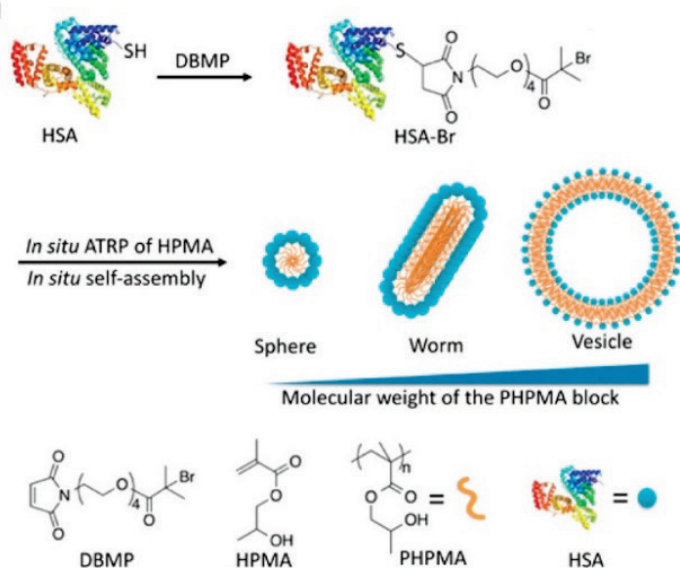
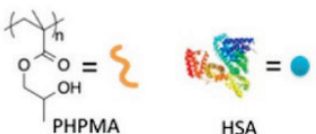

b

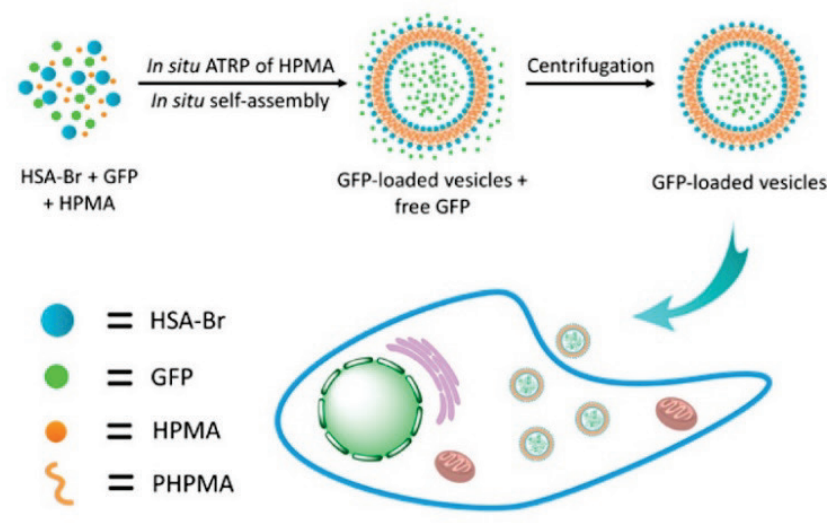

Figure 26. Schematic illustration of: (a) in situ growth of HSA-PHPMA nano-objects with tunable morphologies and (b) in situ encapsulation of GFP into HSA-PHPMA vesicles for the enhanced intracellular delivery of GFP. Adapted from ref ${ }^{139}$.

The ATRP moiety can also be genetically encoded into the protein of interest. This was illustrated by sfGFP that comprised an isobutyryl bromide functionality allowing for the synthesis by photoPISA of sfGFP- $b$-PHPMA micelles with an average diameters of $15-48 \mathrm{~nm} .{ }^{140}$

The protein of interest can also be grafted to a RAFT agent, such as for BSA- $b$-PHPMA nanoparticles. ${ }^{138}$ Similar as HSA-PHPMA nanoparticles, BSA maintained more than $80 \%$ of its 
activity and no toxicity of the nanoparticles was observed in NIH3T3 cells up to $15 \mathrm{mg} \cdot \mathrm{mL}^{-1}$. To investigate their capability as drug-carriers, Dox, Nile Red and DNA were independently encapsulated into the nanoparticles during the PISA process, following by concentration dependent protease-triggered release of the loaded molecules. GOx and HRP enzymes were also conjugated to an ATRP initiator and chain extended together to yield co-micelles exhibiting tunable and enhanced cascade activity. ${ }^{141}$

Aptamers, DNAzymes, and $\mathrm{CpG}$ oligodeoxynucleotides are functional nucleic acids (FNAs) that can provide exciting capacities in biomedicines. ${ }^{150}$ FNA-based nanoparticles can prevent degradations of FNAs in blood circulation and improve their stability in physicochemical conditions. The PISA process using modified FNAs as solvophilic moieties also has great potential in tumor targeting and immunotherapy. ${ }^{142}$ Both sgc8- $b$-PHPMA and CpG- $b$-PHPMA nanoparticles with different morphologies were prepared by PISA. They demonstrated enhanced nuclease resistance, greater cellular uptake efficiency and increased bioactivity. The same strategy was also applied to the design of DNA-based nanoparticles using a functional single stranded DNA (ssDNA). ${ }^{143}$ A 19-mer ssDNA (3'-ATC ATC CAC CAT CTC TTT T-5') linked to a RAFT agent was prepared and used for the polymerization of diacetone acrylamide (DAcAAm) and DMA to yield spherical or worm-like micelles in PBS (Figure 27). 


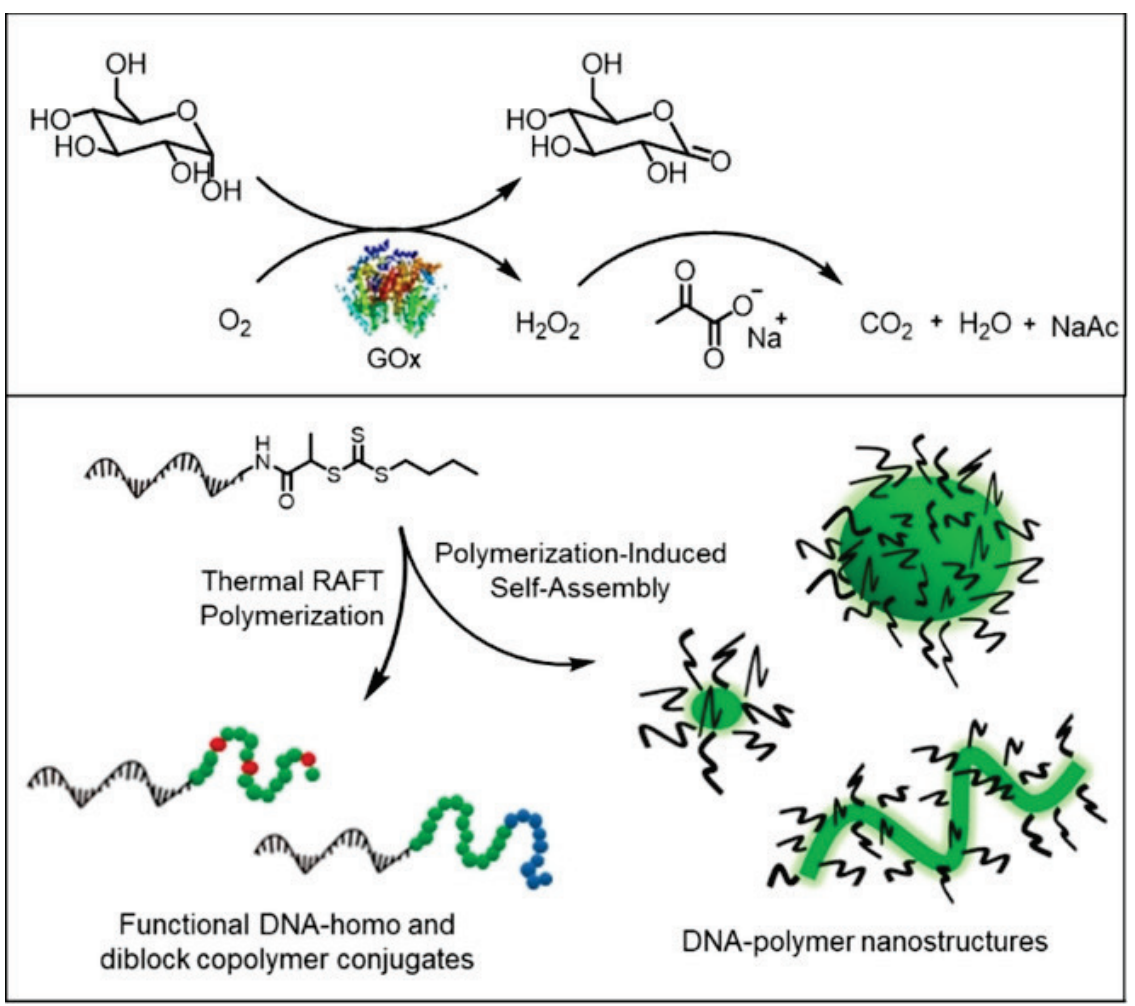

Figure 27. Schematic of the thermal RAFT polymerization from ssDNA under ambient conditions by using enzyme degassing. Adapted from ref. ${ }^{143}$.

Finally, polysaccharides are known for their biocompatibility and show excellent bioactivity, stability and they can be easily functionalized. ${ }^{4,137}$ From the very few examples of PISA systems using polysaccharides as solvophilic block, PMMA or PS was used as the solvophobic block and only spherical shapes were obtained. ${ }^{144-146}$ Direct photo-PISA in presence of modified dextran in water was attempted to yield non-spherical Dex-g5- $b$-PHPMA nanoparticles. ${ }^{147,148}$ 


\section{Crystallization-driven self-assembly (CDSA)}

\section{1. (Bio)degradable nano-objects by CDSA}

\subsubsection{Aliphatic polyester nano-objects}

PLA and PCL are two of the most representative aliphatic polyesters studied for many bio-related applications. ${ }^{5}$ Due to their semi-crystalline mechanical properties, they naturally attracted interest in the design of nano-objects by CDSA. Here we cover bio-relevant applications of CDSA-derived aliphatic polyester nano-objects in aqueous solution.

First example of living CDSA of PLA-containing amphiphilic copolymers was first illustrated by the ROP synthesis of poly(L-LA), poly(D-LA) and poly(rac-LA) macro-CTAs for subsequent RAFT polymerization of a tetrahydropyranyl-acrylate (THPA) monomer, ${ }^{151}$ which yielded a poly(acrylic acid) (PAA) hydrophilic block after deprotection. The self-assembly of the resulting PAA- $b$-PLA diblock copolymers was triggered by heating the polymer in water above the $T_{\mathrm{g}}$ of PLA, to allow core-recrystallization of the nano-objects containing enantiopure homochiral PLA into micelles with cylindrical morphologies. The living epitaxial growth process of the cylindrical micelles was confirmed by re-initiation of cylinder growth via addition of new copolymer. However, atactic PLA core blocks, that were not able to crystallize, afforded spherical micelles. It was also shown that the self-assembly process can proceed during deprotection of PTHPA, thus providing an alternative method and higher final nanoparticle concentrations. ${ }^{152}$ Interestingly, spontaneous structural reorganization of spherical and cylindrical PAA- $b$-PLA diblock copolymer micelles into hollow spheres and cylinders, was also demonstrated upon drying (Figure 29). ${ }^{153}$ These nano-objects have been shown to be able to encapsulate small molecules such as Nile red, which may be of interest for drug delivery. 
a)

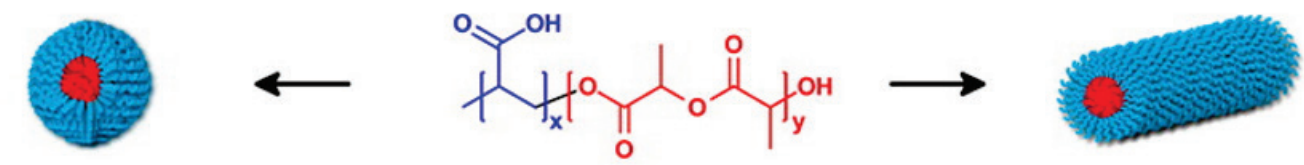

Spherical micelles

Solid core

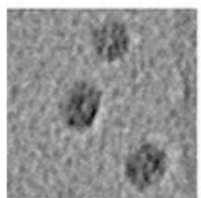

Poly(acrylic acid)- $b$-polylactide

Compartmentalized

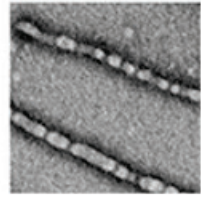

Cylindrical micelles

Hollow core

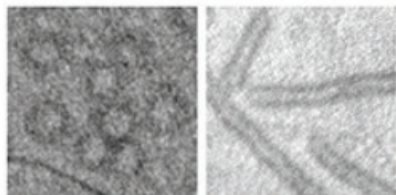

b)

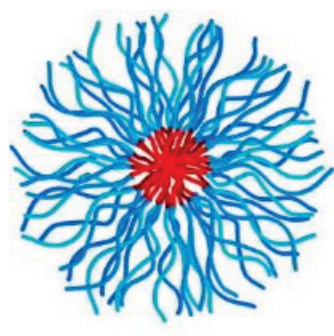

In solution

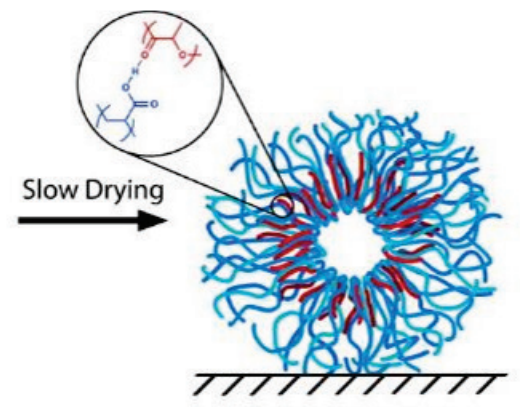

Dried to a surface
Re-suspension

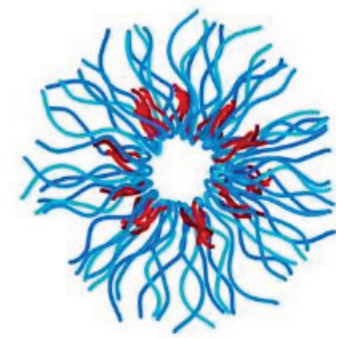

In solution

4

Slow re-equilibration J

Figure 29. (a) Formation of spherical and cylindrical micelles from PAA- $b$-PLA diblock copolymers by CDSA; (b) Schematic representation of hollow nanoparticle formation, showing a cross-section of a cylindrical or spherical particle. Adapted from ref. ${ }^{153}$.

Different functionalization strategies have been studied in order to propose nanoparticles with a biocompatible surface and a semi-crystalline PLA core for biomedical applications. For instance, grafting mannose on the surface of nanoparticles was performed through carbodiimide coupling chemistry to yield glyco-nanoparticles (GNPs) (Figure 30). ${ }^{154}$ Mannose was selected as a typical monosaccharide carbohydrate since it can specifically bind to RAW 264.7 macrophages. Deprotected PDLLA- $b$-PAA spherical micelles and 1D cylindrical micelles were first prepared followed by their post modification with mannose, yielding the nanoparticles without altering their 
colloidal properties. Interestingly, it was revealed that the size and shape of GNP govern their cellular uptake and the inflammatory response. ${ }^{154}$

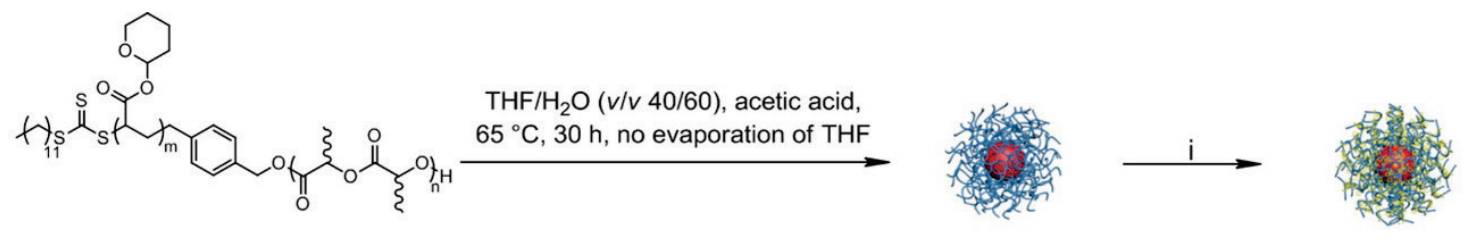

PDLLA-b-PTHPA

PDLLA-b-PAA, Sphere 1

Mannose functionalized Sphere M1

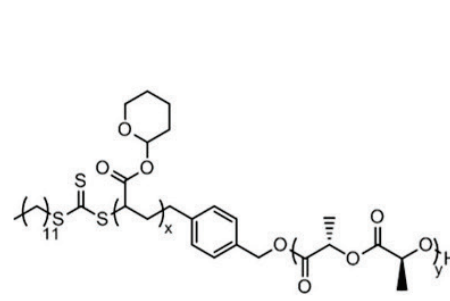

THF/ $\mathrm{H}_{2} \mathrm{O}(v / v 5 / 95)$, acetic acid, $65^{\circ} \mathrm{C}, 30 \mathrm{~h}$, evaporation of THF
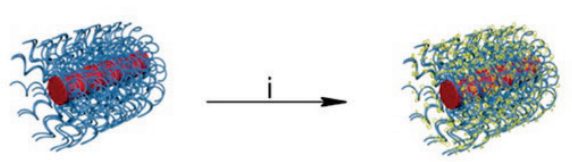

PLLA-b-PAA, Cylinder 2

Mannose functionalized

Cylinder M2

PLLA- $b$-PTHPA

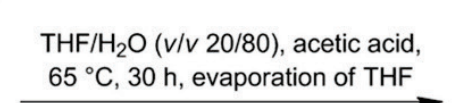

$65^{\circ} \mathrm{C}, 30 \mathrm{~h}$, evaporation of THF

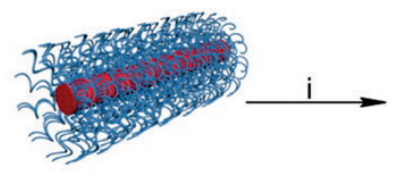

PLLA- $b$-PAA, Cylinder 3

Mannose functionalized

Cylinder M3

i)

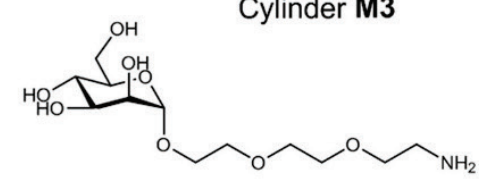

Sulfo-NHS, EDC hydrochloride, $\mathrm{H}_{2} \mathrm{O}$

Figure 30. Preparation of PDLLA- $b$-PAA spherical micelles (1), PLLA- $b$-PAA cylindrical micelles (2) and (3), and their surface functionalization with mannose to yield glyco-nanoparticles (GNPs) M1-M3. Adapted from ref. ${ }^{154}$.

This method was later adapted to the CDSA of pre-synthesised PLLA-based diblock glycopolymers. They were previously conjugated with different saccharides (mannose, galactose, lactose), to yield 1D glyco-cylinder 2D diamond-shaped glyco-platelets (Figure 31). ${ }^{155}$ Not only did assembly conditions and the nature of the carbohydrate play an important role in the selfassembly, but it was also shown that when CDSA was performed under mild degradation conditions, hollow-cored platelets were formed, which is usually a morphology rather difficult to 
obtain. This particular shape was also observed when seeded growth CDSA was used to produce PLLA-based copolymers. ${ }^{156}$

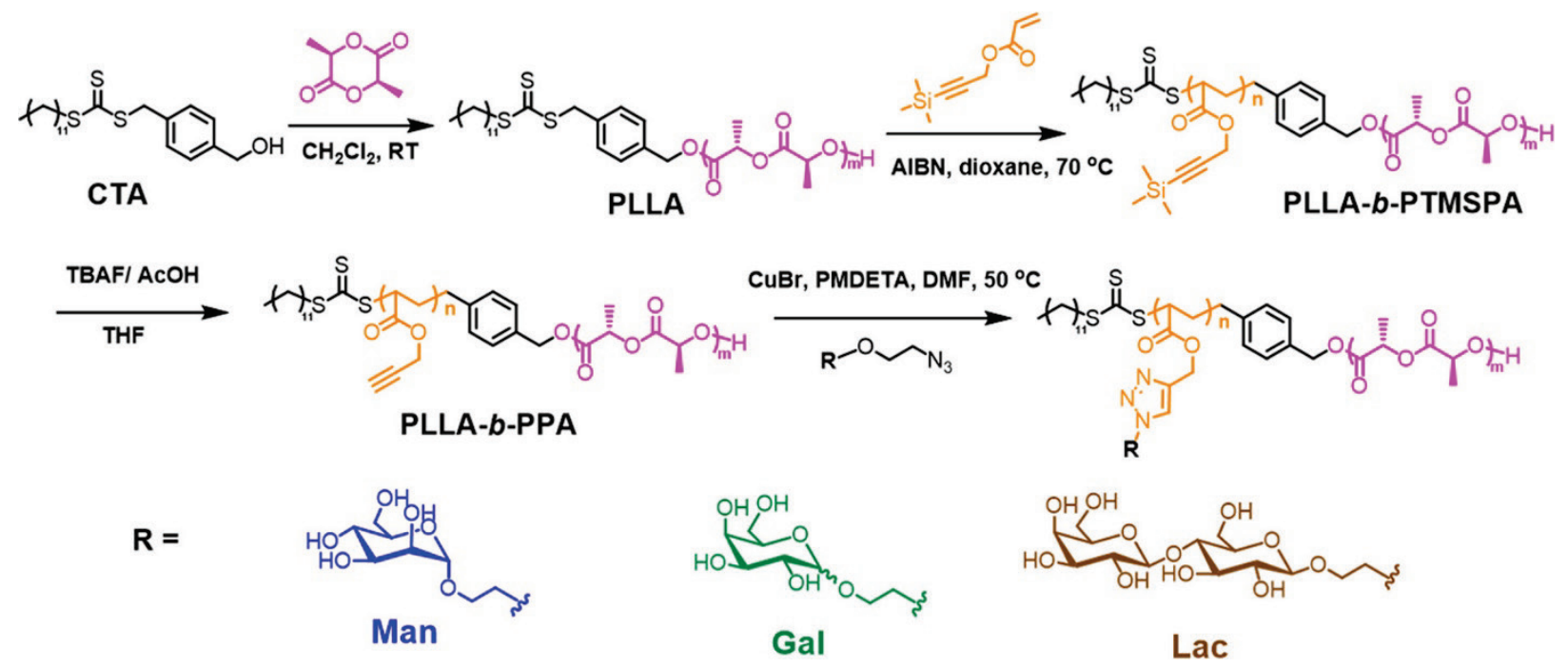

Figure 31. Synthesis scheme of PLLA-based diblock glycopolymers based on mannose, galactose and lactose moieties. Adapted from ref. ${ }^{155}$.

In both cases, the cytotoxicity and cellular uptake of GNPs were evaluated on RAW 264.7 macrophages, resulting no toxicity up to $1 \mathrm{mg} \cdot \mathrm{mL}^{-1}$ for GNPs obtained by post-functionalization and up to $0.1 \mathrm{mg} \cdot \mathrm{mL}^{-1}$ for GNPs formed from pre-synthesized glycopolymers. For the postfunctionalization strategy (Figure 30), spherical GNPs showed greater cellular uptake (via clathrinand caveolin-mediated endocytosis), than cylindrical GNPs, which only followed a clathrinmediated endocytosis mechanism. In terms of inflammatory response, long cylindrical GNPs showed greater inflammatory response than short cylindrical and spherical GNPs. Like for glycocylinders from pre-synthesized GNPs (Figure 31), they exhibited higher cell uptake than 2D glycoplatelets, while large particles were internalized slightly better than small ones. However, glycoplatelets induced a greater inflammatory response than cylindrical GNPs, and smaller glycoplatelets demonstrated higher immune-stimulating efficiency. 
Interestingly, CDSA was also performed from fully degradable amphiphilic diblock copolymers containing a hydrophobic PLA block and a hydrophilic L-cysteine-modified poly $(\alpha-$ D-glucose carbonate) (PDGC-cys) block, that can be degraded hydrolytically and enzymatically. ${ }^{157}$ The obtained PLLA- $b$-PDGC-cys copolymers were synthesized by organocatalyzed ROP followed by grafting with L-cysteine via UV-initiated thiol-yne "click" reaction (Figure 32). A series of spherical, cylindrical and 2D platelet-like bundled cylindrical micelles were obtained. Hydrolytic degradation performed on the nanoparticles at $\mathrm{pH} 1$ and 10 at $37^{\circ} \mathrm{C}$ confirmed their degradation into oligomers. However, the degradation study could not be performed neither at $\mathrm{pH} 7.4$ nor at $\mathrm{pH} 5.0$ because of the precipitation of the copolymers deriving from interactions between carboxylate and ammonium moieties.

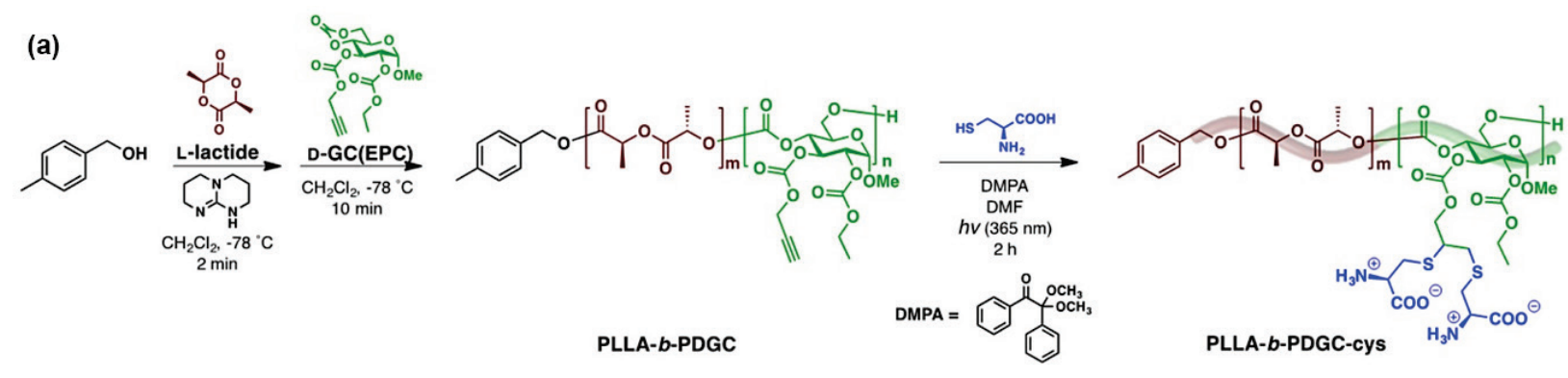

(b)

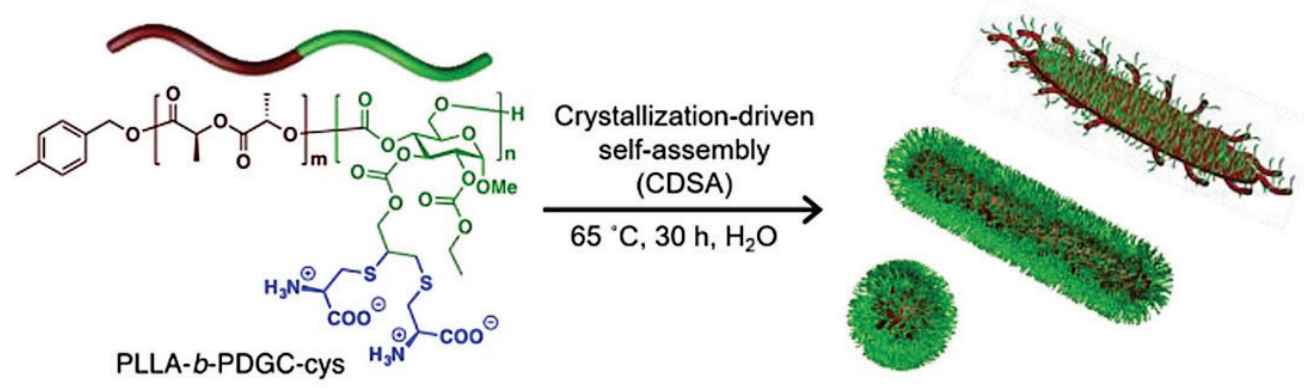

Figure 32. (a) Synthesis of poly(L-lactide)- $b$-poly( $\alpha$ - $D$-glucose carbonate)s (PLLA- $b$-PDGC-cys) copolymers. (b) CDSA of PLLA- $b$-PDGC-cys copolymers to form spherical, cylindrical and 2D plateletlike bundled cylindrical micelles. Adapted from ref. ${ }^{157}$.

Other biocompatible polymers such as poly(4-acryloyl morpholine) (P4AM), poly(ethylene oxide) (PEO) and poly( $N, N$-dimethylacrylamide) (PDMA) were also investigated as hydrophilic corona- 
forming blocks to understand the effect of the polymer hydrophobicity on the morphology of the nanostructures obtained by CDSA. ${ }^{158}$ These copolymers formed cylindrical micelles by CDSA, with the shortest cylinders being obtained for the most hydrophilic corona (PDMA). When applied to poly(dimethyl aminoethyl methacrylate) (PDMAEMA), a charged polymer with antimicrobial activity, spherical or platelet morphologies of different sizes were obtained. It was found that small platelets showed the highest antibacterial activity compared with larger platelets or spherical morphologies. ${ }^{159}$

Even though the degradation of PCL is known to be much slower than that of PLA, its degradation products can be metabolized and cleared by renal excretion. ${ }^{160}$ Similarly to PLAcontaining copolymers, different diblock copolymers based on PCL link to other hydrophilic corona-forming polymers were investigated during CDSA. In a typical example, PEO- $b$-PCL amphiphilic copolymer formed bamboo leaf shaped sheets by CDSA in THF/methanol $\left(L_{\mathrm{n}}=10\right.$ $\mathrm{mm} ; W_{\mathrm{n}}=2 \mathrm{~mm}$ and $\left.D_{\mathrm{n}}=13.5 \mathrm{~nm}\right)$ and remained intact after transfer to water. ${ }^{161}$ Endocytosis of the sheets was studied on five different cell lines, resulting in selective internalization into cells as follows: RAW264.7 cells (92\%), U937 cells (53\%), HUVEC cells (29\%), 293 T cells (5\%) and HeLa cells (3\%). This suggests potential application in precise cell targeting for drug delivery and imaging purposes. The formation of PCL-containing GNPs was also reported by the CDSA process. A PCL macro-CTA was chain extended by a $D$-fructose-functionalized acrylate and BMDO to yield fully degradable copolymers. ${ }^{162}$ The fine-tuning of colloidally stable $2 \mathrm{D}$ platelets was achieved after self-assembly. The degradability of this copolymer was studied on the nanoparticles in the presence of Pseudomonas $s p$. lipase ( $\geq 22$ units/mg) in PBS, and it showed complete degradation by DLS and SEC. ${ }^{163}$

In most examples of CDSA, a water-miscible organic solvent is necessary to induce the living growth of nano-objects. Solvent switch or evaporation is therefore required to obtain aqueous suspensions of the nano-objects, which may limit their controlled growth and affect their morphology. ${ }^{35}$ Performing CDSA in water would not only simplify the entire process, but also allow for more precise control of particle growth. In this context, CDSA was successfully 
implemented in either alcoholic or aqueous media using PCL-based triblock copolymers. ${ }^{164}$ For aqueous CDSA, different concentrations of PCL50- $b$-PMMA $20-b-\mathrm{PDMA}_{200}$ unimers in acetone were added to preformed seeds made from same copolymer in water (Figure 33a). Rapid evaporation of acetone allowed the formation of stable micelles in aqueous media. Linear epitaxial growth with a predictable based on the unimer-to-seed ratio was demonstrated (Figure 33b-i). No cytotoxicity was observed with cylindrical micelles up to $5 \mathrm{mg} \cdot \mathrm{mL}^{-1}$ on $\mathrm{MC} 3 \mathrm{~T} 3$ (murine preosteoclasts) and A549 (human lung cancer fibroblasts) cell lines via MTT assays. The precise control in dimensions achieved in water were then exploited to create biologically relevant hydrogel materials by incorporating MC3T3 and A549 cells into a preformed hydrogel with 15 wt. $\%$ solids content obtained following the same protocol, resulting in $>95 \%$ cell livingness. In a different study, PDMA- $b$-PCL- $b$-PDMA triblock copolymers formed well-defined 1D or 2D crystallized structures like fibers, platelets or long cylinders by CDSA, depending on the solubility of the core-forming block in the solvent selected. ${ }^{165}$ 


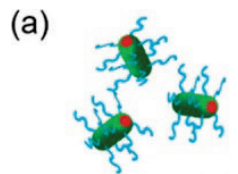

PCL-b-PMMA-b-PDMA seeds
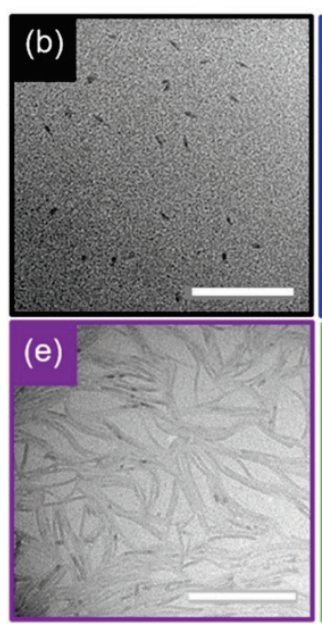

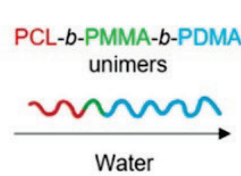

Water

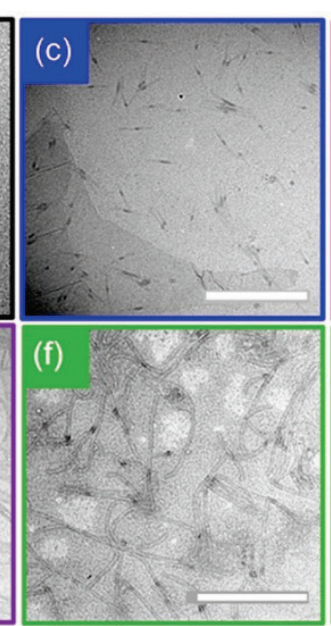

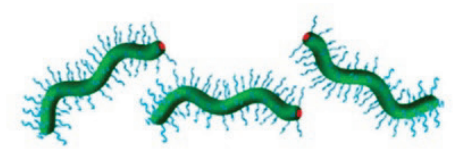

Monodisperse cylinders in water

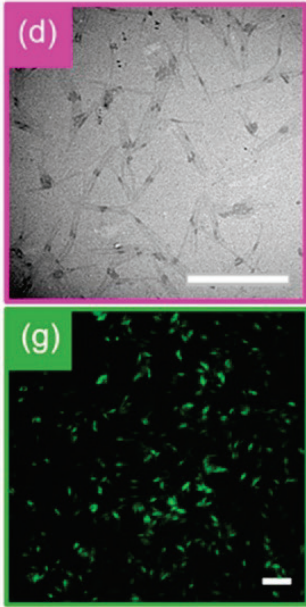

(h)

(i)

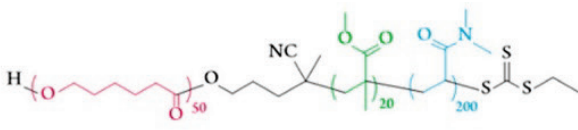

PCL-b-PMMA-b-PDMA

\begin{tabular}{cccc}
\hline Target $(\mathrm{nm})$ & $L_{\mathrm{w}}$ & $L_{\mathrm{n}}$ & $L_{\mathrm{w}} / L_{\mathrm{n}}$ \\
\hline 100 & 111 & 103 & 1.08 \\
300 & 297 & 265 & 1.12 \\
500 & 513 & 473 & 1.09 \\
800 & 826 & 757 & 1.09 \\
\hline
\end{tabular}

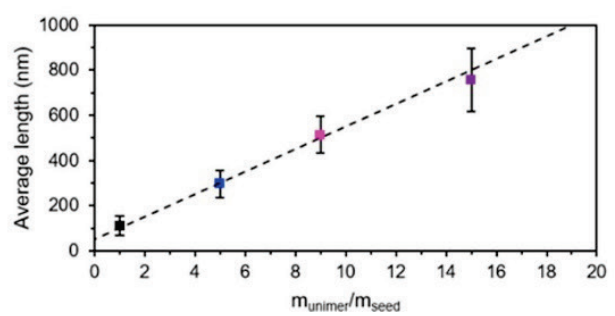

Figure 33. (a) Synthesis by CDSA of cylinders by epitaxial growth in water using $\mathrm{PCL}_{50}-b-\mathrm{PMMA}_{20}-b$ PDMA 200 triblock copolymers. (b-e) TEM images of cylinders grown with a unimer/seed ratio of: (b) 1; (c) 5; (d) 9 and (e) 15. Scale bar $=1000 \mathrm{~nm}$. (f and g) TEM (scale bar = $1000 \mathrm{~nm}$ ) and confocal microscope (scale bar $=20 \mu \mathrm{m}$ ) images of fluorescent cylinders formed with a unimer/seed ratio of 15 . (h) Length dispersity of cylinders. (i) Plot representing the linear epitaxial growth of cylinders. Adapted from ref. ${ }^{164}$.

Combining hybrid materials and PCL-containing nanoparticles from CDSA may also be advantageous. ${ }^{166}$ PCL- $b$-P4VP copolymer was employed here as a seed anchored at the surface of carbon nanotubes (CNTs), followed by in situ CDSA via addition of PCL- $b$-PDMA unimers in ethanol. It was demonstrated that presence of CNTs exerted a spatial confinement for the epitaxial growth of PCL-b-PDMA, which led to long and thin platelets. Without CNTs, 2D platelets were formed. The cytocompatibility of such hybrid hierarchical nanoassemblies in aqueous suspensions was demonstrated on mouse fibroblast (L929) cells from 0 to $140 \mu \mathrm{g} . \mathrm{mL}^{-1}$ over $24 \mathrm{~h}$, proving their potential for biomedical applications.

In terms of drug delivery, encapsulation of drugs into a crystalline core is challenging because it can disrupt crystallinity and lead to particle disassembly. To circumvent this issue, drugs and other functional molecules can be incorporated onto the surface of crystalline particles in order 
to maintain the crystallinity of the core. ${ }^{167}$ This was illustrated by the synthesis of biodegradable 2D glycoplatelets prepared by CDSA and surface-incorporation of Dox or cyanine 5 (Cy5) as a fluorescent dye (Figure 34), either on the preformed polymer or via post-functionalization. This new strategy may open new perspectives in the use of crystalline core nano-objects in drug delivery applications.
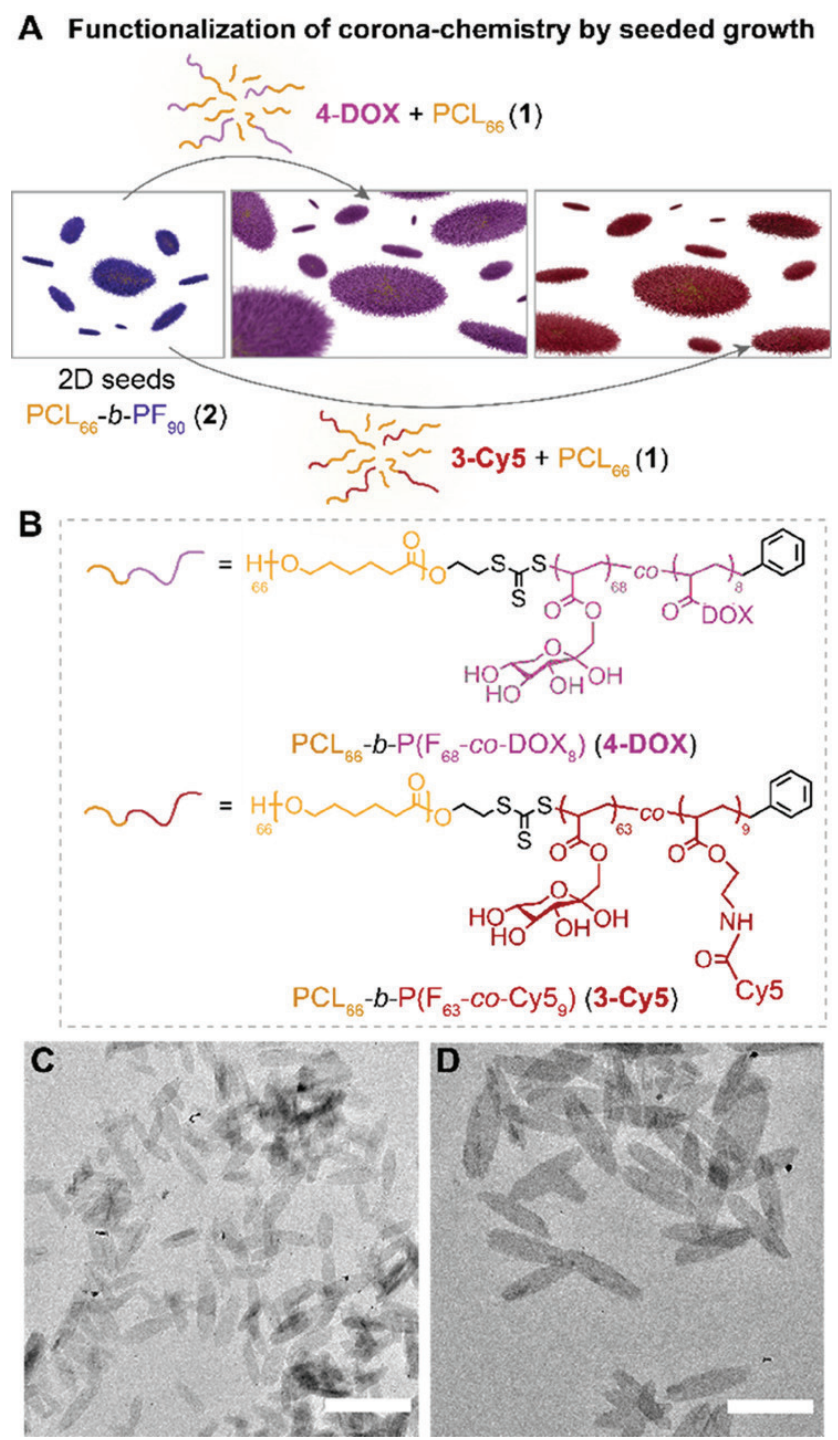

Figure 34. (A) Schematic representation of surface functionalization of glycoplatelets by seeded growth with Dox-conjugated and Cy5-conjugated block copolymers. (B) Chemical structures of block copolymers 3-Cy5 and 4-Dox. TEM images of the platelet particles prepared by seeded growth of $\mathrm{PCL}_{66}-b-\mathrm{PF}_{90}$ (2) seed crystallites using unimeric blends of (C) 3-Cy5/PCL 66 and (D) 4-Dox/PCL 66 . Scale bar $=5 \mu \mathrm{m}$. Adapted from ref. ${ }^{167}$. 


\subsubsection{Aliphatic polycarbonate nano-objects}

In addition to polyesters, aliphatic polycarbonates have also emerged as an interesting class of polymers due to their low inherent toxicity and their biodegradability. ${ }^{5}$ For instance, poly(trimethylene carbonate) (PTMC) and its derivatives have been extensively studied. A poly(spiro[fluorene-9,5'-[1,3]-dioxan]-2'-one)- $b$-poly(ethylene glycol) (PFTMC- $b$-PEG) diblock copolymer synthesized by organocatalytic ROP was described. ${ }^{168}$ It was subjected to living CDSA to form well-defined fiber-like micelles with controlled length $(\sim 40-1500 \mathrm{~nm})$ and then safely transferred to water. TEM, AFM, SAXS, and WAXS analyses indicated rectangular cross-section arrangement of the crystallized PFTMC core surrounded by a PEG corona. Their cytotoxicity was evaluated by two different assays to access the cell viability and reductive metabolism in two representative cell lines (WI-38 fetal lung fibroblast cells and HeLa cervical cancer cells). Even if these needle-like nano-objects were not cytotoxic up to $0.1 \mathrm{mg} \cdot \mathrm{mL}^{-1}$, it was hypothesized that they might disrupt the extracellular matrix of the cells. The living CDSA process was also successful extended to PFTMC- $b$-poly(2-vinylpyridine) (PFTMC- $b$-P2VP). Interestingly, addition of PFTMC- $b$-P2VP to PFTMC- $b$-PEG seed micelles resulted in the formation of well-defined segmented fibers.

\subsubsection{Polypeptoid-based nano-objects}

Recent years have witnessed the growth of polypeptoid-based nanomaterials by CDSA. For instance, poly(ethylene glycol)- $b$-poly( $N$-octylglycine) (PEG- $b$-PNOG), poly(ethylene glycol)- $b$ $\operatorname{poly}(N$-(2-phenylethyl)glycine) (PEG- $b$-PNPE) and poly( $N$-methyl glycine)- $b$-poly $(N$-decyl glycine) (PNMG- $b$-PNDG) diblock copolymers were self-assembled into various hierarchical structures such as 1D wormlike nanofibrils, 1D rigid nanorods and 2D nanosheets. ${ }^{169-172}$ Interestingly, changing the nature of the $N$-substituent (from $N$-octylglycine to $N$-2-ethyl-1hexylglycine) allowed to change the hierarchical self-assembly of diblock copolypeptoids (from microflower morphology to hexagonal nanosheets, respectively). ${ }^{169}$ Other polypeptoid-based 
diblock copolymers were also synthesized: (i) poly(ethylene glycol)-b-poly( $N$-(2phenylethyl)glycine) (PEG- $b$-PNPE) in order to investigate the effect of the polymer solubility on the nanoassemblies ${ }^{173}$ and (ii) poly( $N$-alkylglycine)- $b$-poly( $N$-2-(2-methoxyethoxy)ethylglycine) to explore the selective assembly of sequence-defined amphiphilic diblock copolypeptoids. ${ }^{174}$

Polypeptoids can also exhibit tunable semi-crystalline properties and a thermo-responsive behavior. ${ }^{55,56}$ There have been a few successful attempts to build thermo-responsive polypeptoid nanomaterials by CDSA. ${ }^{175-177}$ In this context, a thiol-terminated triethylene glycol monomethyl ether $\left(\mathrm{EG}_{3}\right)$ was conjugated to a poly( $N$-allylglycine)-b-poly( $N$-octylglycine) (PNAG- $b$-PNOG)

copolymer to yield amphiphilic (PNAG- $g$-EG 3 )- $b$-PNOG copolymers. ${ }^{175}$ They exhibited a reversible thermoresponsive behavior but an irreversible sphere-to-cylinder transition upon heating-cooling cycle due to the presence of the crystalline PNOG block. Cellular uptake experiments on HeLa, RAW264.7 and L929 cells showed a greater internalization of the cylinders compared to that of the spherical ones.

\subsection{Biocompatible nano-objects by CDSA}

\subsubsection{Poly(2-oxazoline)-based nano-objects}

Even if they are supposedly non-biodegradable, ${ }^{178}$ intensive efforts have been devoted to the development of functionalized POx-based materials for bio-applications owing to their noncytotoxicity and 'stealth' properties. ${ }^{179}$ The first example of living CDSA of POx was reported in 2015. ${ }^{176}$ Poly(2-methyl-2-oxazoline) (PMeOx) was used as the hydrophilic block and poly(2isopropyl-2-oxazoline) (PiPrOx) was the hydrophobic block because of its structural similarity with poly( $N$-isopropylacrylamide) (PNIPAM). It also exhibits a LCST behavior in water around body temperature and is capable of crystallizing above its LCST. The self-assembly of the PMeOx$b$-PiPrOx copolymer was performed by heating at $65^{\circ} \mathrm{C}$ in water followed by rapid cooling. Stable spherical and distorted crystallized micelles or micron-size fibers were obtained when the heating 
proceeded over $90 \mathrm{~min}$. The biological evaluation of similar PMeOx- $b$-PiPrOx copolymers was investigated by preparing nanorods with length ranging from 60 to $635 \mathrm{~nm}$, whose control over their growth was achieved by CDSA in water (Figure 35a-c). ${ }^{177}$ Despite simultaneous nanoparticle fragmentation and dissolution upon fiber sonication, as well as slow nanorod disassembly in water, preliminary investigation confirmed their low association with blood cells (Figure 35d) and their stealth properties via pharmacokinetics and ex vivo biodistribution studies in mice, during which promising blood circulation lifetimes $(10 \%$ injected dose per gram of tissue remained in the blood stream after $24 \mathrm{~h}$ ) and systemic clearance mainly via the liver were observed (Figure 35e). However, no significant influence of the length of the nanorod properties was witnessed.

a)
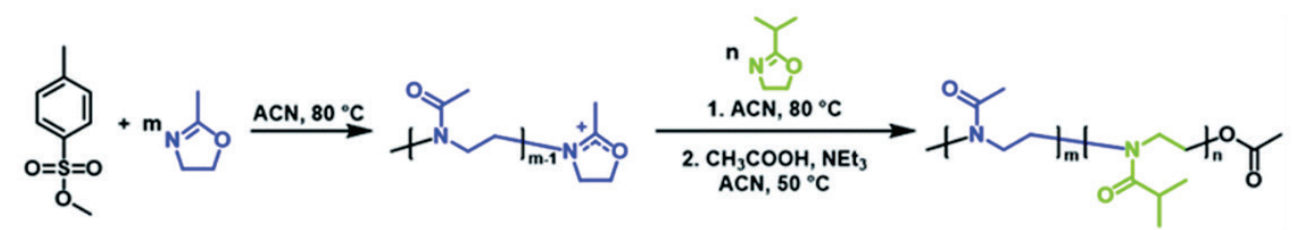

b)

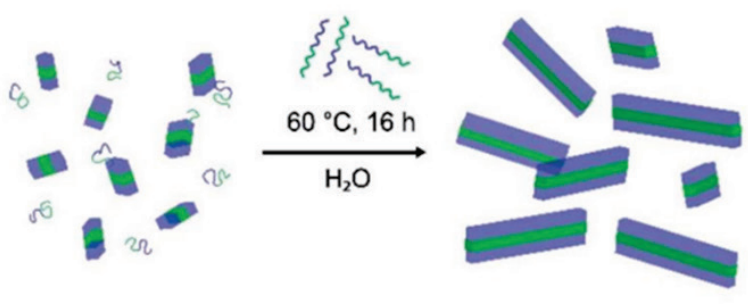

d)

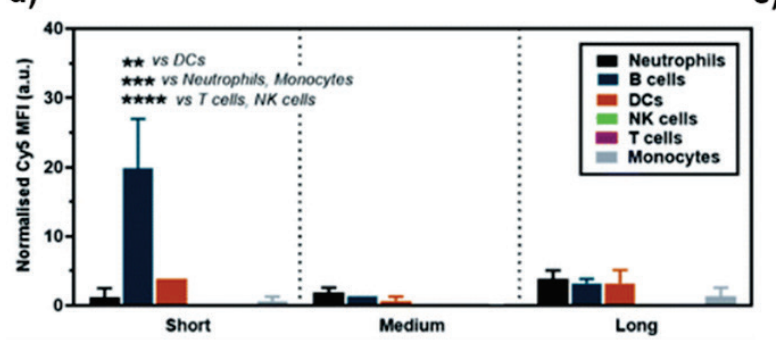

c)

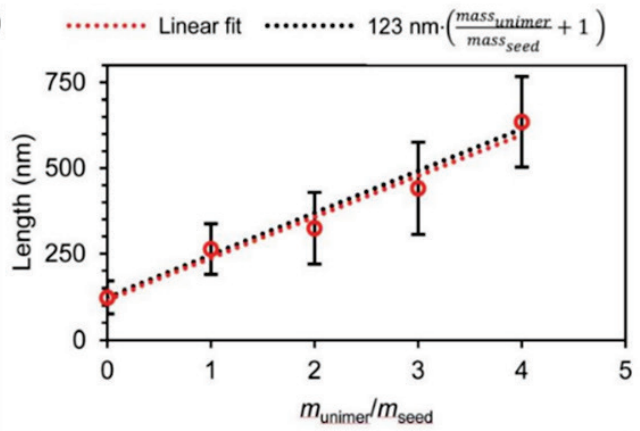

e)

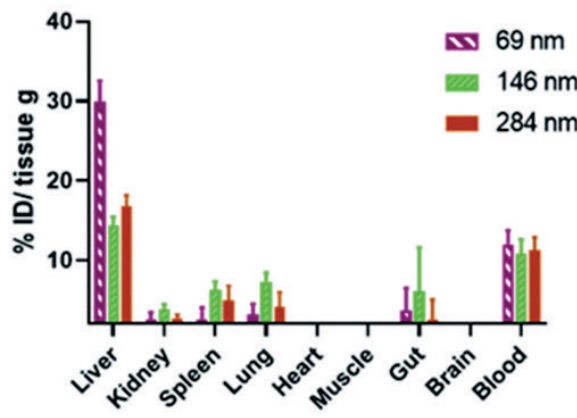

Figure 35. (a) Synthesis of $\mathrm{PMeOx}_{\mathrm{m}}-b$-PiPrOx $\mathrm{x}_{\mathrm{n}}$ block copolymers. (b) CDSA of PMeOx $\mathrm{x}_{\mathrm{m}}-b$-PiPrOx $\mathrm{x}_{\mathrm{n}}$ block copolymers in water. (c) Linear control over rod-like micelle growth. (d) Human blood immune cell association assay of rod-like micelles with different lengths. (e) Ex vivo biodistribution study of $\mathrm{PMeOx}_{\mathrm{m}}{ }^{-}$ $b$-PiPrOx $x_{n}$ nanorods in mice. Adapted from ref. ${ }^{177}$. 
Dual responsive POx-based crystalline nanomaterials were also developed by CDSA (Figure 36). ${ }^{180}$ Poly(2-acrylamido glycolic acid) (PAGA) was used as hydrophilic polyelectrolyte block and its ability to chelate metal ions. ${ }^{181}$ This was successfully applied in aqueous solution to different metal ions such as $\mathrm{Co}, \mathrm{Ni}$ and $\mathrm{Cu}$, resulting in well-defined spherical or wormlike hybrid nanomaterials.

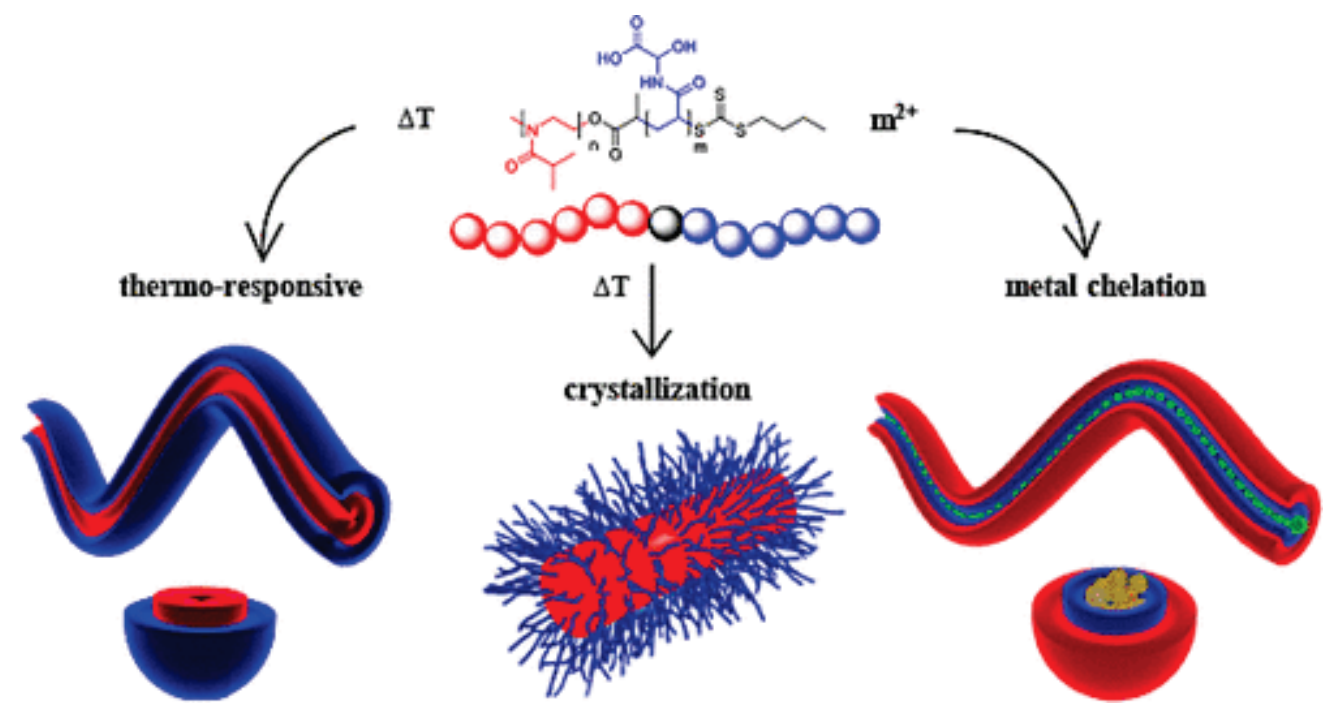

Figure 36. Synthesis of dual stimuli-responsive poly(2-iso-propyl-2-oxazoline)- $b$-poly(2-acrylamido glycolic acid) (PiPrOx- $b$-PAGA) crystalline nanomaterials by CDSA. Adapted from ref. ${ }^{180}$.

\subsubsection{PEG-based polymers as the solvophilic block}

PEGylated nano-objects with non-biodegradable core-forming polymers have also been synthesized by CDSA. Such systems mainly served to study the influence of the nano-object morphology on cell uptake and cytotoxicity. For instance, diblock copolymers comprising a POEGMA solvophilic block plus a few (aminopropyl)methacrylamide (APMA) units and a polyferrocenylsilane (PFS) hydrophobic block produced narrowly dispersed rod-like micelles of variable lengths $(80-2000 \mathrm{~nm})$ by CDSA. ${ }^{182}$ The rod-like micelles maintained their colloid stability in PBS after at least 3 months and showed no cytotoxicity on human breast cancer cell lines (MDA-MB-231 and MDA-MB-436) up to $0.1 \mathrm{mg} \cdot \mathrm{mL}^{-1}$. The successful conjugation of 
diethylenetriaminepentaacetic acid (DTPA) as a metal chelator to APMA moieties was demonstrated and further applied to the capture of heavy metals such as $\mathrm{Tb}^{3+}$ ion. A follow-up study on their cell uptake behavior and penetration into multicellular tumor spheroids compared $\mathrm{PFS}_{27}-b-\mathrm{P}\left(\mathrm{APMA}_{3}-\mathrm{co}-\mathrm{OEGMA}_{48}\right)$ rigid rod-like micelles with analogous spherical micelles prepared by nanoprecipitation. ${ }^{183}$ An in vitro 3D model of multicellular tumor spheroids (MCTSs) with an average diameter of $500 \mu \mathrm{m}$ for both cell lines was used to simulate their behavior in vivo. Overall, it was found that rod-like micelles can penetrate deeper into tumor models than spherical micelles, but longer rod-like micelles decreased the uptake and penetration into MDA-MB-436 cells. This gave a glance on how rod-like nanocarriers with narrow length distributions behave in 3D tumor models and possibly in vivo. In another example, PEGylated copolymers were used as seed nanofibers in CDSA to investigate their internalization by cells. ${ }^{184}$ Functionalized poly(dihexylfluorene)- $b$-poly(ethylene glycol) $\left(\mathrm{PDHF}_{13}-b-\mathrm{PEG}_{227}\right)$ was used as seed to grow with $\mathrm{PDHF}_{13}-b-\mathrm{PEG}_{227}-\mathrm{BODIPY}(\mathrm{BD})$ and $\mathrm{PDHF}_{13}-b-\mathrm{PEG}_{227}$-folic acid (FA) unimers to yield C-BA-B-C pentablock 1D nanofibers (Figure 37a,b). After their transfer into water without altering their colloid characteristics (Figure 37c), the pentablock nanofibers showed no cytotoxicity on HeLa cancer cells and WI-38 fetal lung fibroblasts up to $100 \mu \mathrm{g} \cdot \mathrm{mL}^{-1}$, as well as FA receptormediated cell internalization by HeLa cells (Figure 37d). Interestingly, it was shown that nanofibers were located on the cell membrane, and that only those positioned in an 'end-on' fashion were internalized, highlighting the unique uptake mechanism of high aspect ratio 1D nanomaterials. Small nanoparticle fragments $(\sim 20 \mathrm{~nm}$ long), likely resulting from fiber dissociation were noticed inside the cells, which could be seen as a new drug delivery approach. 
a)

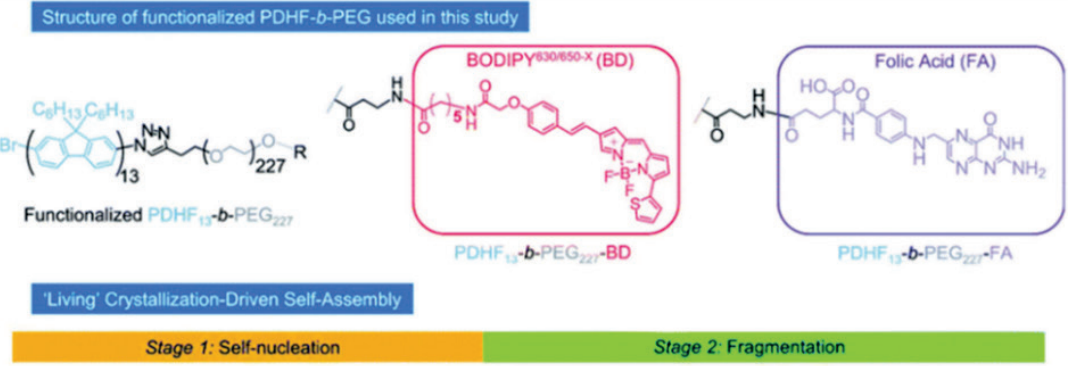

b)

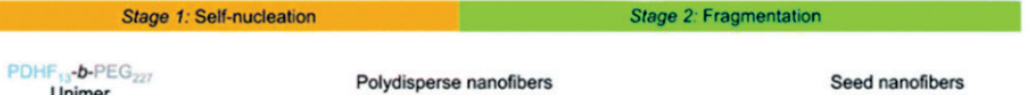

Polydisperse nanofibers

Seed nanofibers
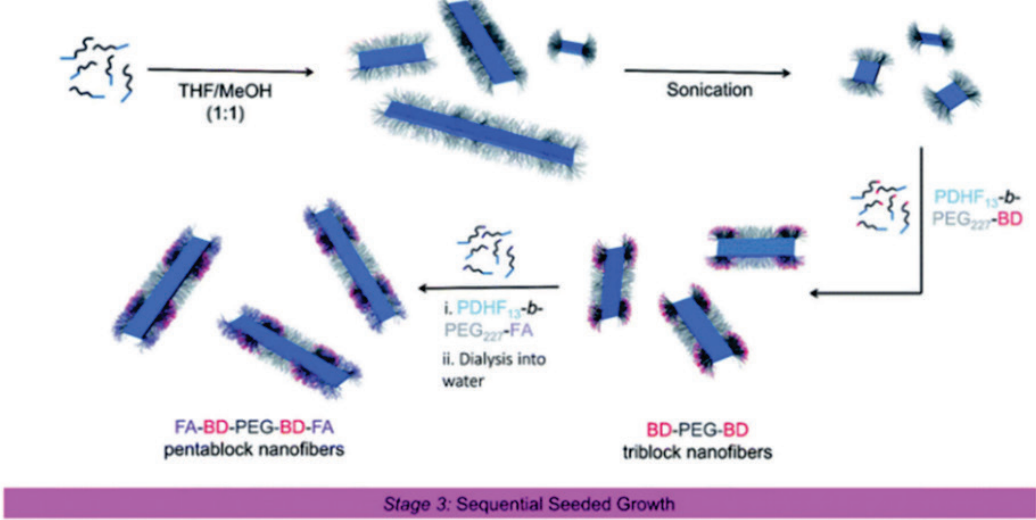

c)

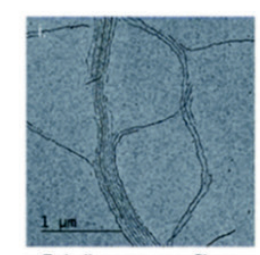

Polydisperse nanofibers

d)

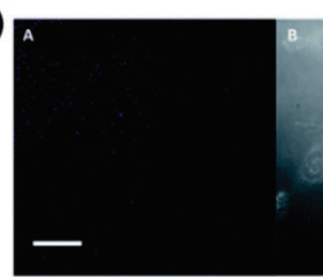
in THFMeOH (1:1)
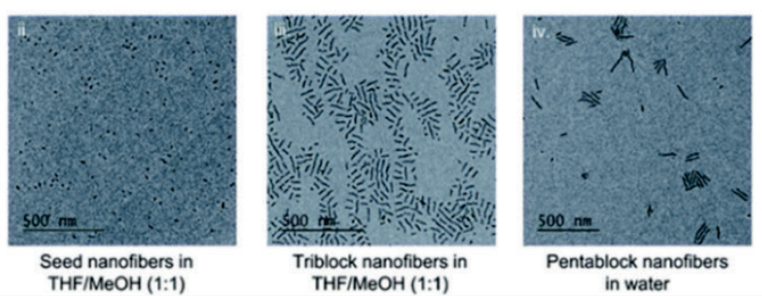

THF/MeOH (1:1)
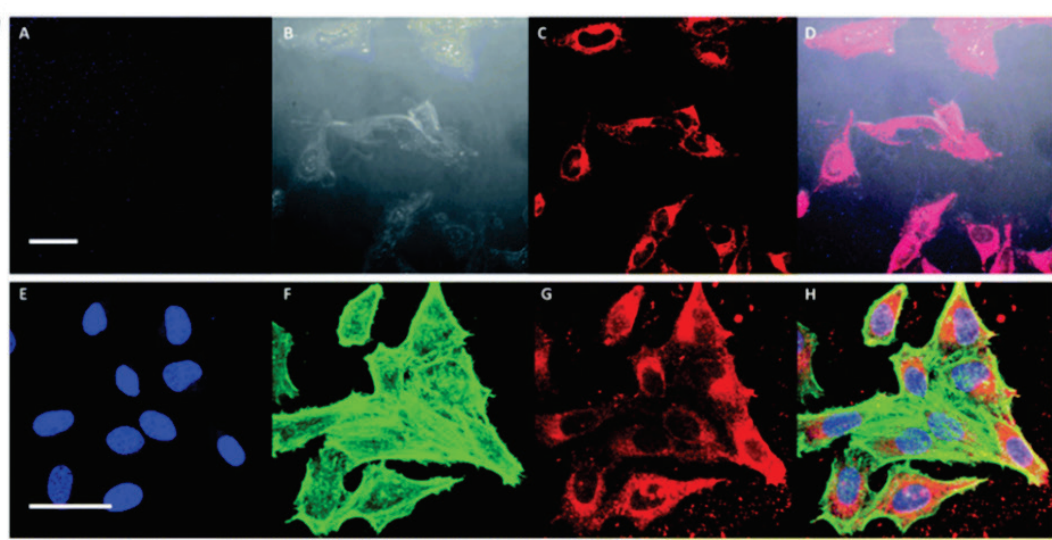

Figure 37. (a) Synthesis of poly(dihexylfluorene)- $b$-poly(ethylene glycol) $\left(\mathrm{PDHF}_{13}-b-\mathrm{PEG}_{227}\right)$ seed, and $\mathrm{PDHF}_{13}-b-\mathrm{PEG}_{227}-\mathrm{BODIPY}(\mathrm{BD})$ and $\mathrm{PDHF}_{13}-b-\mathrm{PEG}_{227}$-folic acid (FA) unimers. (b) CDSA process for the preparation of pentablock 1D nanofibers. (c) TEM images of formed nanofibers in different solvents. (d) Confocal microscope image of HeLa cells after incubation with FA-BD-PEG-BD-FA pentablock nanofibers (A-D) after $30 \mathrm{~min}$ and (E-H) after $1 \mathrm{~h}$. Adapted from ref. ${ }^{184}$. 
By a combination of anionic polymerization and photoinitiated thiol-ene "click" chemistry, another study used poly(ferrocenyldimethylsilane)-b-poly(allyl glycidyl ether) grafted with triethylene glycol (PFS- $b$-(PEO-g-TEG)), to generate cylindrical micelles in aqueous media with narrow length distributions by CDSA. ${ }^{39}$ Formation of B-A-B triblock comicelles were obtained by extension of PFS- $b$-(PEO-g-TEG) cylindrical micelles using PFS- $b$-P2VP copolymers. To highlight their potential in biomedical applications, a selective complexation of DNA by the positively charged domains was illustrated.

\subsubsection{Other biocompatible polymers as the solvophilic block}

In addition to PEG, other biocompatible polymers have also been used in CDSA. For instance, poly(glycosyloxyethyl methacrylate) (PGlcEMA), a bioactive methacrylate ester-based polymer carrying glycolate side chains, was selected to produce multicompartment patchy nanoparticles by CDSA as cargoes for drug delivery purposes (Figure 38). ${ }^{185}$ In this study, PGlcEMA94- $b$-PBzA $277^{-}$ $b$-P4VP 370 terpolymer nanoparticles were formed by a two-step hierarchical self-assembly procedure that allowed to separately encapsulate two types of fluorescent molecules (Dox, Cy5) in different nanoparticle compartments. The nanoparticles were cytocompatible up to $200 \mathrm{ug} \cdot \mathrm{mL}^{-}$ ${ }^{1}$ with MCF-7 cancer cells while the Dox-loaded nanoparticles gave an IC50 value of $15.3 \mathrm{ug} . \mathrm{mL}^{-}$

1. Successful delivery of both fluorophores and establishment of FRET signals were proven inside the cells, providing another way to achieve simultaneous and traceable delivery. 
a)

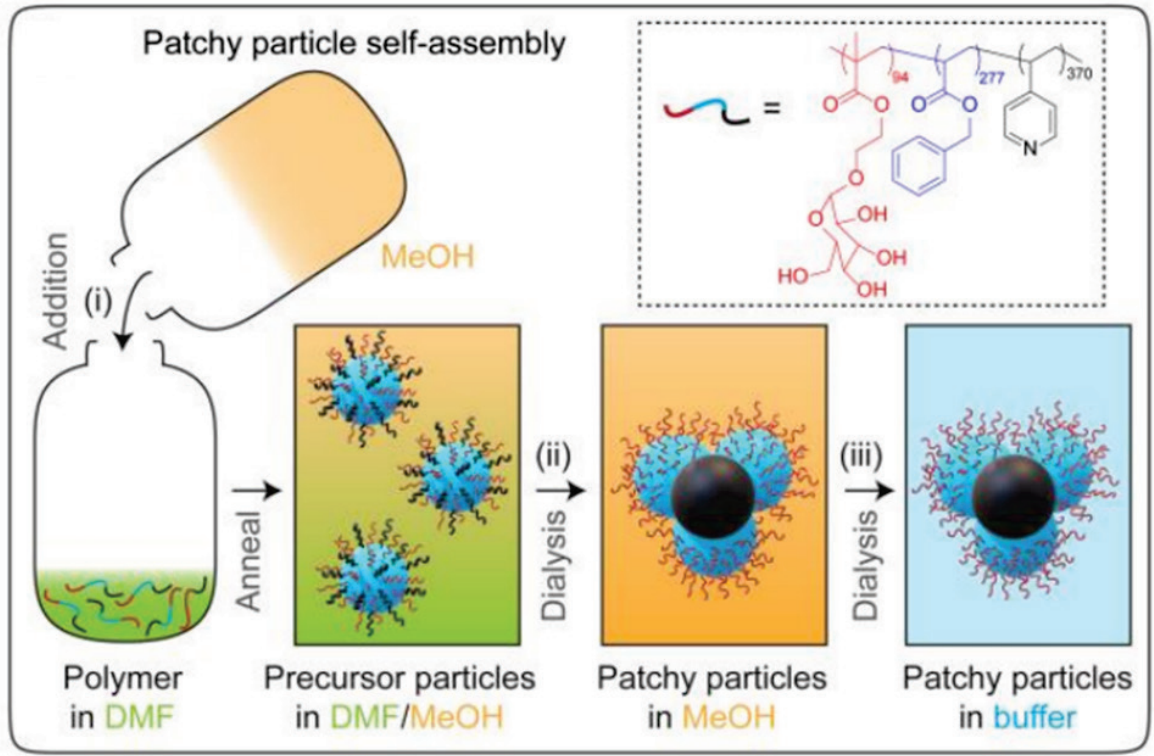

b)

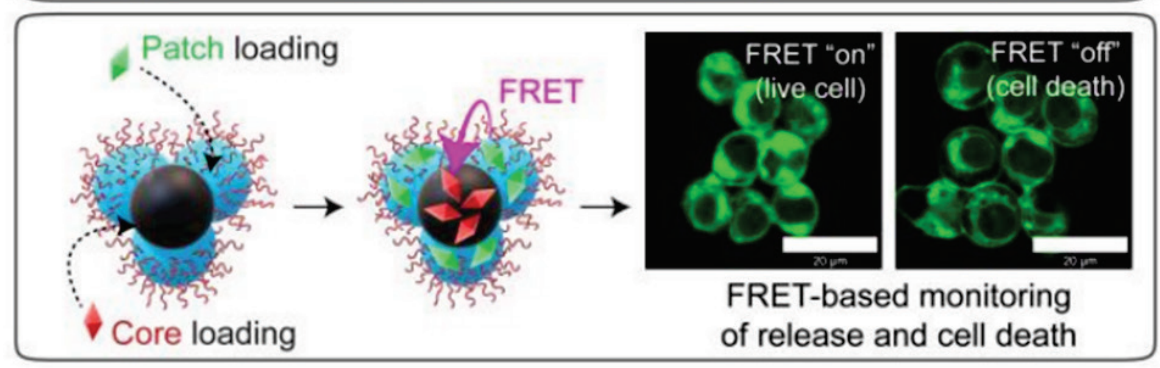

Figure 38. (a) Synthesis of linear $\mathrm{PGlcEMA}_{94}-b-\mathrm{PBzA}_{277}-b-\mathrm{P}_{4 \mathrm{VP}} \mathrm{P}_{30}$ terpolymer nanoparticles and transfer of nanoparticles to buffer solution. (b) selective co-loading into nanoparticles proved by both fluorophores and release of FRET signals. Adapted from ref. ${ }^{185}$.

Silicone polymers, also called polysiloxanes, are also known as biocompatible polymers. ${ }^{186}$ In this context, poly(ferrocenyldimethylsilane)- $b$-poly(methylvinylsiloxane) (PFS- $b$-PMVS) diblock copolymer cylindrical micelles were prepared by living CDSA (Figure 39). ${ }^{40}$ Unimers bearing BODIPY or biotin moieties grafted on the PMVS block were also incorporated into the cylinders for signaling or post binding purposes. Their formulation into water/alcohol mixtures led to hierarchically ordered colloidosomes that can be further transferred to water and crosslinked for biomimetic encapsulation, drug delivery or biosensing properties. 


\section{Conclusion}

In this review, we covered the advances in the design, synthesis and evaluation of diblock copolymer nano-objects synthesized by PISA or CDSA processes, with a strict focus on (bio)degradable and biocompatible systems, which is of tremendous importance when biomedical applications are envisioned. Those systems were mainly composed of a (bio)degradable core and/or a biocompatible shell.

In recent years, considerable efforts have been made to produce nano-objects from PISA and CDSA that meet these criteria. While degradability often relied on insertion of ester, amide or disulfide bonds in the core-forming polymer backbone, biocompatibility was conferred by the use of biocompatible shell-forming polymers such as PEG, PEG derivatives and well-established PEG alternatives, including biomacromolecules. However, despite many advances and promising results, it appears that PISA systems often lack biodegradability compared to CDSA-derived systems given their polyester core, even though some progress has been made in this direction, especially with the recent development of rROPISA and PISA from NCAs. This can be explained by the fact that CDSA relies on the self-assembly of preformed diblock copolymers whereas in PISA, the copolymers are synthesized in situ, which is much more challenging with water-sensitive monomers like CKAs and/or when labile ester groups should be incorporated in the targeted structures. Despite their biodegradable core, drug encapsulation into CDSA systems is also not straightforward despite promising achievements.

Regarding the degradability of PISA systems, an improvement could result from the design and use of new monomers that are tolerant to aqueous conditions, so that labile groups can be easily inserted into the copolymer backbones to achieve efficient (bio)degradability. As this area of research is very active, there is no doubt that the coming years will see further progress and significant achievements that will bring PISA and CDSA systems closer to clinical translation. 


\section{References}

1. Soppimath, K. S.; Aminabhavi, T. M.; Kulkarni, A. R.; Rudzinski, W. E. Biodegradable Polymeric Nanoparticles as Drug Delivery Devices. J. Control. Release. 2001, 70 (1-2), 1-20.

2. Vert, M.; Doi, Y.; Hellwich, K.-H.; Hess, M.; Hodge, P.; Kubisa, P.; Rinaudo, M.; Schué, F. Terminology for biorelated polymers and applications (IUPAC Recommendations 2012). Pure Appl. Chem. 2012, 84 (2), 377-410.

3. Anderson, J. M. Biocompatibility. In Polymer Science: A Comprehensive Reference; Elsevier, 2012; pp 363-383.

4. Nicolas, J.; Mura, S.; Brambilla, D.; Mackiewicz, N.; Couvreur, P. Design, Functionalization Strategies and Biomedical Applications of Targeted Biodegradable/Biocompatible Polymer-Based Nanocarriers for Drug Delivery. Chem. Soc. Rev. 2013, 42 (3), 1147-1235.

5. Seyednejad, H.; Ghassemi, A. H.; van Nostrum, C. F.; Vermonden, T.; Hennink, W. E. Functional Aliphatic Polyesters for Biomedical and Pharmaceutical Applications. J. Control. Release. 2011, 152 (1), 168-176.

6. Deming, T. J. Synthetic Polypeptides for Biomedical Applications. Prog. Polym. Sci. 2007, 32 (8), $858-875$.

7. Nicolas, J.; Couvreur, P. Synthesis of Poly(Alkyl Cyanoacrylate)-Based Colloidal Nanomedicines. WIREs Nanomedicine Nanobiotechnology. 2009, 1 (1), 111-127.

8. Veronese, F. M. Peptide and Protein PEGylation: A Review of Problems and Solutions. Biomaterials. 2001, 22 (5), 405-417.

9. Duncan, R. The Dawning Era of Polymer Therapeutics. Nat. Rev. Drug Discov. 2003, 2 (5), 347-360.

10. Roberts, M. J.; Bentley, M. D.; Harris, J. M. Chemistry for Peptide and Protein PEGylation. Adv. Drug Deliv. Rev. 2012, 64, 116-127.

11. Stolnik, S.; Illum, L.; Davis, S. S. Long Circulating Microparticulate Drug Carriers. Adv. Drug Deliv. Rev. 1995, 16 (2-3), 195-214.

12. Knop, K.; Hoogenboom, R.; Fischer, D.; Schubert, U. S. Poly(Ethylene Glycol) in Drug Delivery: Pros and Cons as Well as Potential Alternatives. Angew. Chem. Int. Ed. 2010, 49 (36), 6288-6308.

13. Abdelhamid, D.; Arslan, H.; Zhang, Y.; Uhrich, K. E. Role of Branching of Hydrophilic Domain on Physicochemical Properties of Amphiphilic Macromolecules. Polym. Chem. 2014, 5 (4), 1457-1462.

14. Miao, T.; Wang, J.; Zeng, Y.; Liu, G.; Chen, X. Polysaccharide-Based Controlled Release Systems for Therapeutics Delivery and Tissue Engineering: From Bench to Bedside. Adv. Sci. 2018, 5 (4), 1700513.

15. Barz, M.; Luxenhofer, R.; Zentel, R.; Vicent, M. J. Overcoming the PEG-Addiction: Well-Defined Alternatives to PEG, from Structure-Property Relationships to Better Defined Therapeutics. Polym. Chem. 2011, 2 (9), 1900.

16. Mai, Y.; Eisenberg, A. Self-Assembly of Block Copolymers. Chem. Soc. Rev. 2012, 41 (18), 5969. 
17. Rösler, A.; Vandermeulen, G. W. M.; Klok, H.-A. Advanced Drug Delivery Devices via SelfAssembly of Amphiphilic Block Copolymers. Adv. Drug Deliv. Rev. 2012, 64, 270-279.

18. Warren, N. J.; Armes, S. P. Polymerization-Induced Self-Assembly of Block Copolymer NanoObjects via RAFT Aqueous Dispersion Polymerization. J. Am. Chem. Soc. 2014, 136 (29), 1017410185.

19. Derry, M. J.; Fielding, L. A.; Armes, S. P. Polymerization-Induced Self-Assembly of Block Copolymer Nanoparticles via RAFT Non-Aqueous Dispersion Polymerization. Prog. Polym. Sci. 2016, 52, 1-18.

20. Penfold, N. J. W.; Yeow, J.; Boyer, C.; Armes, S. P. Emerging Trends in Polymerization-Induced SelfAssembly. ACS Macro Lett. 2019, 8 (8), 1029-1054.

21. D’Agosto, F.; Rieger, J.; Lansalot, M. RAFT-Mediated Polymerization-Induced Self-Assembly. Angew. Chem. Int. Ed. 2020, 59 (22), 8368-8392.

22. Braunecker, W. A.; Matyjaszewski, K. Controlled/Living Radical Polymerization: Features, Developments, and Perspectives. 50 Years Living Polym. 2007, 32 (1), 93-146.

23. Nicolas, J.; Guillaneuf, Y.; Lefay, C.; Bertin, D.; Gigmes, D.; Charleux, B. Nitroxide-Mediated Polymerization. Top. Issue Polym. Chem. 2013, 38 (1), 63-235.

24. Perrier, S. 50th Anniversary Perspective: RAFT Polymerization-A User Guide. Macromolecules. 2017, 50 (19), 7433-7447.

25. Matyjaszewski, K.; Xia, J. Atom Transfer Radical Polymerization. Chem. Rev. 2001, 101 (9), 29212990.

26. Charleux, B.; Delaittre, G.; Rieger, J.; D’Agosto, F. Polymerization-Induced Self-Assembly: From Soluble Macromolecules to Block Copolymer Nano-Objects in One Step. Macromolecules. 2012, 45 (17), 6753-6765.

27. Canning, S. L.; Smith, G. N.; Armes, S. P. A Critical Appraisal of RAFT-Mediated PolymerizationInduced Self-Assembly. Macromolecules. 2016, 49 (6), 1985-2001.

28. Wang, X.; An, Z. New Insights into RAFT Dispersion Polymerization-Induced Self-Assembly: From Monomer Library, Morphological Control, and Stability to Driving Forces. Macromol. Rapid Commun. 2019, 40 (2), 1800325.

29. Foster, J. C.; Varlas, S.; Couturaud, B.; Jones, J. R.; Keogh, R.; Mathers, R. T.; O’Reilly, R. K. Predicting Monomers for Use in Polymerization-Induced Self-Assembly. Angew. Chem. Int. Ed. 2018, 57 (48), 15733-15737.

30. Khor, S. Y.; Quinn, J. F.; Whittaker, M. R.; Truong, N. P.; Davis, T. P. Controlling Nanomaterial Size and Shape for Biomedical Applications via Polymerization-Induced Self-Assembly. Macromol. Rapid Commun. 2019, 40 (2), 1800438.

31. Le, D.; Keller, D.; Delaittre, G. Reactive and Functional Nanoobjects by Polymerization-Induced Self-Assembly. Macromol. Rapid Commun. 2019, 40 (2), 1800551.

32. Phan, H.; Taresco, V.; Penelle, J.; Couturaud, B. Polymerisation-Induced Self-Assembly (PISA) as a Straightforward Formulation Strategy for Stimuli-Responsive Drug Delivery Systems and 
Biomaterials: Recent Advances. Biomater. Sci. 2021, 9 (1), 38-50.

33. Zhang, W.-J.; Hong, C.-Y.; Pan, C.-Y. Polymerization-Induced Self-Assembly of Functionalized Block Copolymer Nanoparticles and Their Application in Drug Delivery. Macromol. Rapid Commun. 2019, 40 (2), 1800279.

34. Gurnani, P.; Perrier, S. Controlled Radical Polymerization in Dispersed Systems for Biological Applications. Prog. Polym. Sci. 2020, 102, 101209.

35. MacFarlane, L.; Zhao, C.; Cai, J.; Qiu, H.; Manners, I. Emerging Applications for Living Crystallization-Driven Self-Assembly. Chem. Sci. 2021, 12 (13), 4661-4682.

36. Ganda, S.; Stenzel, M. H. Concepts, Fabrication Methods and Applications of Living CrystallizationDriven Self-Assembly of Block Copolymers. Prog. Polym. Sci. 2020, 101, 101195.

37. Foster, J. C.; Varlas, S.; Couturaud, B.; Coe, Z.; O’Reilly, R. K. Getting into Shape: Reflections on a New Generation of Cylindrical Nanostructures' Self-Assembly Using Polymer Building Blocks. $J$. Am. Chem. Soc. 2019, 141 (7), 2742-2753.

38. Pearce, A. K.; Wilks, T. R.; Arno, M. C.; O’Reilly, R. K. Synthesis and Applications of Anisotropic Nanoparticles with Precisely Defined Dimensions. Nat. Rev. Chem. 2021, 5 (1), 21-45.

39. Nazemi, A.; Boott, C. E.; Lunn, D. J.; Gwyther, J.; Hayward, D. W.; Richardson, R. M.; Winnik, M. A.; Manners, I. Monodisperse Cylindrical Micelles and Block Comicelles of Controlled Length in Aqueous Media. J. Am. Chem. Soc. 2016, 138 (13), 4484-4493.

40. Dou, H.; Li, M.; Qiao, Y.; Harniman, R.; Li, X.; Boott, C. E.; Mann, S.; Manners, I. Higher-Order Assembly of Crystalline Cylindrical Micelles into Membrane-Extendable Colloidosomes. Nat. Commun. 2017, 8 (1), 426.

41. Jin, X.-H.; Price, M. B.; Finnegan, J. R.; Boott, C. E.; Richter, J. M.; Rao, A.; Menke, S. M.; Friend, R. H.; Whittell, G. R.; Manners, I. Long-Range Exciton Transport in Conjugated Polymer Nanofibers Prepared by Seeded Growth. Science. 2018, 360 (6391), 897-900.

42. Qian, J.; Li, X.; Lunn, D. J.; Gwyther, J.; Hudson, Z. M.; Kynaston, E.; Rupar, P. A.; Winnik, M. A.; Manners, I. Uniform, High Aspect Ratio Fiber-like Micelles and Block Co-Micelles with a Crystalline $\pi$-Conjugated Polythiophene Core by Self-Seeding. J. Am. Chem. Soc. 2014, 136 (11), 4121-4124.

43. Agarwal, S. Chemistry, Chances and Limitations of the Radical Ring-Opening Polymerization of Cyclic Ketene Acetals for the Synthesis of Degradable Polyesters. Polym. Chem. 2010, 1 (7), 953.

44. Delplace, V.; Nicolas, J. Degradable Vinyl Polymers for Biomedical Applications. Nat. Chem. 2015, 7 (10), 771-784.

45. Pesenti, T.; Nicolas, J. 100th Anniversary of Macromolecular Science Viewpoint: Degradable Polymers from Radical Ring-Opening Polymerization: Latest Advances, New Directions, and Ongoing Challenges. ACS Macro Lett. 2020, 9 (12), 1812-1835.

46. Tardy, A.; Nicolas, J.; Gigmes, D.; Lefay, C.; Guillaneuf, Y. Radical Ring-Opening Polymerization: Scope, Limitations, and Application to (Bio)Degradable Materials. Chem. Rev. 2017, 117 (3), 13191406. 
47. Guegain, E.; Zhu, C.; Giovanardi, E.; Nicolas, J. Radical Ring-Opening Copolymerization-Induced Self-Assembly (RROPISA). Macromolecules. 2019, 52 (10), 3612-3624.

48. Fielding, L. A.; Derry, M. J.; Ladmiral, V.; Rosselgong, J.; Rodrigues, A. M.; Ratcliffe, L. P. D.; Sugihara, S.; Armes, S. P. RAFT Dispersion Polymerization in Non-Polar Solvents: Facile Production of Block Copolymer Spheres, Worms and Vesicles in n-Alkanes. Chem. Sci. 2013, 4 (5), 2081-2087.

49. Zhu, C.; Nicolas, J. Towards Nanoparticles with Site-Specific Degradability by Ring-Opening Copolymerization Induced Self-Assembly in Organic Medium. Polym. Chem. 2021, 12 (4), 594-607.

50. Zhu, C.; Denis, S.; Nicolas, J. A Simple Route to Aqueous Suspensions of Degradable Copolymer Nanoparticles Based on Radical Ring-Opening Polymerization-Induced Self-Assembly (rROPISA). Chem. Mater. 2022. DOI: 10.1021/acs.chemmater.1c04151

51. Guégain, E.; Michel, J.-P.; Boissenot, T.; Nicolas, J. Tunable Degradation of Copolymers Prepared by Nitroxide-Mediated Radical Ring-Opening Polymerization and Point-by-Point Comparison with Traditional Polyesters. Macromolecules. 2018, 51 (3), 724-736.

52. Ratcliffe, L. P. D.; Couchon, C.; Armes, S. P.; Paulusse, J. M. J. Inducing an Order-Order Morphological Transition via Chemical Degradation of Amphiphilic Diblock Copolymer NanoObjects. Biomacromolecules. 2016, 17 (6), 2277-2283.

53. Jiang, J.; Zhang, X.; Fan, Z.; Du, J. Ring-Opening Polymerization of N-Carboxyanhydride-Induced Self-Assembly for Fabricating Biodegradable Polymer Vesicles. ACS Macro Lett. 2019, 8 (10), $1216-1221$.

54. Grazon, C.; Salas-Ambrosio, P.; Ibarboure, E.; Buol, A.; Garanger, E.; Grinstaff, M. W.; Lecommandoux, S.; Bonduelle, C. Aqueous Ring-Opening Polymerization-Induced Self-Assembly (ROPISA) of N-Carboxyanhydrides. Angew. Chem. Int. Ed. 2020, 59 (2), 622-626.

55. Zhang, D.; Lahasky, S. H.; Guo, L.; Lee, C.-U.; Lavan, M. Polypeptoid Materials: Current Status and Future Perspectives. Macromolecules. 2012, 45 (15), 5833-5841.

56. Chan, B. A.; Xuan, S.; Li, A.; Simpson, J. M.; Sternhagen, G. L.; Yu, T.; Darvish, O. A.; Jiang, N.; Zhang, D. Polypeptoid Polymers: Synthesis, Characterization, and Properties. Biopolymers. 2018, 109 (1), e23070.

57. Birke, A.; Ling, J.; Barz, M. Polysarcosine-Containing Copolymers: Synthesis, Characterization, Self-Assembly, and Applications. Prog. Polym. Sci. 2018, 81, 163-208.

58. Lowe, S.; O’Brien-Simpson, N. M.; Connal, L. A. Antibiofouling Polymer Interfaces: Poly(Ethylene Glycol) and Other Promising Candidates. Polym. Chem. 2015, 6 (2), 198-212.

59. Settanni, G.; Schäfer, T.; Muhl, C.; Barz, M.; Schmid, F. Poly-Sarcosine and Poly(Ethylene-Glycol) Interactions with Proteins Investigated Using Molecular Dynamics Simulations. Comput. Struct. Biotechnol. J. 2018, 16, 543-550.

60. Varlas, S.; Georgiou, P. G.; Bilalis, P.; Jones, J. R.; Hadjichristidis, N.; O’Reilly, R. K. Poly(Sarcosine)-Based Nano-Objects with Multi-Protease Resistance by Aqueous Photoinitiated Polymerization-Induced Self-Assembly (Photo-PISA). Biomacromolecules. 2018, 19 (11), 44534462. 
61. Ke, P. C.; Lin, S.; Parak, W. J.; Davis, T. P.; Caruso, F. A Decade of the Protein Corona. ACS Nano. 2017, 11 (12), 11773-11776.

62. Beck-Broichsitter, M.; Nicolas, J.; Couvreur, P. Design Attributes of Long-Circulating Polymeric Drug Delivery Vehicles. Eur. J. Pharm. Biopharm. 2015, 97, 304-317.

63. Warren, N. J.; Mykhaylyk, O. O.; Mahmood, D.; Ryan, A. J.; Armes, S. P. RAFT Aqueous Dispersion Polymerization Yields Poly(Ethylene Glycol)-Based Diblock Copolymer Nano-Objects with Predictable Single Phase Morphologies. J. Am. Chem. Soc. 2014, 136 (3), 1023-1033.

64. Tan, J.; Sun, H.; Yu, M.; Sumerlin, B. S.; Zhang, L. Photo-PISA: Shedding Light on PolymerizationInduced Self-Assembly. ACS Macro Lett. 2015, 4 (11), 1249-1253.

65. Blackman, L. D.; Varlas, S.; Arno, M. C.; Houston, Z. H.; Fletcher, N. L.; Thurecht, K. J.; Hasan, M.; Gibson, M. I.; O'Reilly, R. K. Confinement of Therapeutic Enzymes in Selectively Permeable Polymer Vesicles by Polymerization-Induced Self-Assembly (PISA) Reduces Antibody Binding and Proteolytic Susceptibility. ACS Cent. Sci. 2018, 4 (6), 718-723.

66. He, J.; Cao, J.; Chen, Y.; Zhang, L.; Tan, J. Thermoresponsive Block Copolymer Vesicles by Visible Light-Initiated Seeded Polymerization-Induced Self-Assembly for Temperature-Regulated Enzymatic Nanoreactors. ACS Macro Lett. 2020, 9 (4), 533-539.

67. Blackman, L. D.; Varlas, S.; Arno, M. C.; Fayter, A.; Gibson, M. I.; O’Reilly, R. K. Permeable Protein-Loaded Polymersome Cascade Nanoreactors by Polymerization-Induced Self-Assembly. ACS Macro Lett. 2017, 6 (11), 1263-1267.

68. Blackman, L. D.; Oo, Z. Y.; Qu, Y.; Gunatillake, P. A.; Cass, P.; Locock, K. E. S. Antimicrobial Honey-Inspired Glucose-Responsive Nanoreactors by Polymerization-Induced Self-Assembly. ACS Appl. Mater. Interfaces. 2020, 12 (10), 11353-11362.

69. Tan, J.; Liu, D.; Bai, Y.; Huang, C.; Li, X.; He, J.; Xu, Q.; Zhang, L. Enzyme-Assisted Photoinitiated Polymerization-Induced Self-Assembly: An Oxygen-Tolerant Method for Preparing Block Copolymer Nano-Objects in Open Vessels and Multiwell Plates. Macromolecules. 2017, 50.

70. Liu, X.; Sun, M.; Sun, J.; Hu, J.; Wang, Z.; Guo, J.; Gao, W. Polymerization Induced Self-Assembly of a Site-Specific Interferon $\alpha$-Block Copolymer Conjugate into Micelles with Remarkably Enhanced Pharmacology. J. Am. Chem. Soc. 2018, 140 (33), 10435-10438.

71. Varlas, S.; Blackman, L. D.; Findlay, H. E.; Reading, E.; Booth, P. J.; Gibson, M. I.; O’Reilly, R. K. Photoinitiated Polymerization-Induced Self-Assembly in the Presence of Surfactants Enables Membrane Protein Incorporation into Vesicles. Macromolecules. 2018, 51 (16), 6190-6201.

72. Ding, Y.; Zhang, X.; Xu, Y.; Cheng, T.; Ou, H.; Li, Z.; An, Y.; Shen, W.; Liu, Y.; Shi, L. Polymerization-Induced Self-Assembly of Large-Scale Iohexol Nanoparticles as Contrast Agents for X-Ray Computed Tomography Imaging. Polym. Chem. 2018, 9 (21), 2926-2935.

73. Fan, B.; Liu, Y.; Wan, J.; Crawford, S.; Thang, S. H. Polymerization-Induced Self-Assembly (PISA) and "Host-Guest" Complexation-Directed Polymer/Gold Nanocomposites. ACS Mater. Lett. 2020, 2 (5), 492-498.

74. Chen, M.; Li, J.-W.; Zhang, W.-J.; Hong, C.-Y.; Pan, C.-Y. PH- and Reductant-Responsive Polymeric Vesicles with Robust Membrane-Cross-Linked Structures: In Situ Cross-Linking in Polymerization- 
Induced Self-Assembly. Macromolecules. 2019, 52 (3), 1140-1149.

75. Droumaguet, B. L.; Nicolas, J. Recent Advances in the Design of Bioconjugates from Controlled/Living Radical Polymerization. Polym. Chem. 2010, 1 (5), 563-598.

76. Pelegri-O'Day, E. M.; Lin, E.-W.; Maynard, H. D. Therapeutic Protein-Polymer Conjugates: Advancing Beyond PEGylation. J. Am. Chem. Soc. 2014, 136 (41), 14323-14332.

77. Roy, D.; Brooks, W. L. A.; Sumerlin, B. S. New Directions in Thermoresponsive Polymers. Chem. Soc. Rev. 2013, 42 (17), 7214-7243.

78. Zhang, W.-J.; Hong, C.-Y.; Pan, C.-Y. Fabrication of Reductive-Responsive Prodrug Nanoparticles with Superior Structural Stability by Polymerization-Induced Self-Assembly and Functional Nanoscopic Platform for Drug Delivery. Biomacromolecules. 2016, 17 (9), 2992-2999.

79. Karagoz, B.; Boyer, C.; Davis, T. P. Simultaneous Polymerization-Induced Self-Assembly (PISA) and Guest Molecule Encapsulation. Macromol. Rapid Commun. 2014, 35 (4), 417-421.

80. Karagoz, B.; Esser, L.; Duong, H. T.; Basuki, J. S.; Boyer, C.; Davis, T. P. Polymerization-Induced Self-Assembly (PISA) - Control over the Morphology of Nanoparticles for Drug Delivery Applications. Polym. Chem. 2013, 5 (2), 350-355.

81. Zhou, Y.; Wang, Z.; Wang, Y.; Li, L.; Zhou, N.; Cai, Y.; Zhang, Z.; Zhu, X. Azoreductase-Triggered Fluorescent Nanoprobe Synthesized by RAFT-Mediated Polymerization-Induced Self-Assembly for Drug Release. Polym. Chem. 2020, 11 (35), 5619-5629.

82. Wang, Y.; Yang, D.; Hu, Y.; Wang, Y.; Yang, W. J.; Wang, L. Synthesis of Water-Soluble EuropiumContaining Nanoprobes via Polymerization-Induced Self-Assembly and Their Cellular Imaging Applications. Talanta. 2021, 122182.

83. Delplace, V.; Couvreur, P.; Nicolas, J. Recent Trends in the Design of Anticancer Polymer Prodrug Nanocarriers. Polym. Chem. 2014, 5 (5), 1529-1544.

84. Kaga, S.; Truong, N. P.; Esser, L.; Senyschyn, D.; Sanyal, A.; Sanyal, R.; Quinn, J. F.; Davis, T. P.; Kaminskas, L. M.; Whittaker, M. R. Influence of Size and Shape on the Biodistribution of Nanoparticles Prepared by Polymerization-Induced Self-Assembly. Biomacromolecules. 2017, 18 (12), 3963-3970.

85. Kumar, G. S.; Neckers, D. C. Photochemistry of Azobenzene-Containing Polymers. Chem. Rev. 1989, 89 (8), 1915-1925.

86. Pitto-Barry, A. Polymers and Boron Neutron Capture Therapy (BNCT): A Potent Combination. Polym. Chem. 2021, 12 (14), 2035-2044.

87. Sherlock Huang, L.-C.; Le, D.; Hsiao, I.-L.; Fritsch-Decker, S.; Hald, C.; Huang, S.-C.; Chen, J.-K.; Ru Hwu, J.; Weiss, C.; Hsu, M.-H.; Delaittre, G. Boron-Rich, Cytocompatible Block Copolymer Nanoparticles by Polymerization-Induced Self-Assembly. Polym. Chem. 2021, 12 (1), 50-56.

88. Kopeček, J.; Kopečková, P. HPMA Copolymers: Origins, Early Developments, Present, and Future. Adv. Drug Deliv. Rev. 2010, 62 (2), 122-149.

89. Zhang, W.-J.; Hong, C.-Y.; Pan, C.-Y. Efficient Fabrication of Photosensitive Polymeric NanoObjects via an Ingenious Formulation of RAFT Dispersion Polymerization and Their Application for 
Drug Delivery. Biomacromolecules. 2017, 18 (4), 1210-1217.

90. Zhang, W.-J.; Hong, C.-Y.; Pan, C.-Y. Artificially Smart Vesicles with Superior Structural Stability: Fabrication, Characterizations, and Transmembrane Traffic. ACS Appl. Mater. Interfaces. 2017, 9 (17), 15086-15095.

91. Zhao, X.; Chen, M.; Zhang, W.-G.; Wang, C.-H.; Wang, F.; You, Y.-Z.; Zhang, W.-J.; Hong, C.-Y. Polymerization-Induced Self-Assembly to Produce Prodrug Nanoparticles with ReductionResponsive Camptothecin Release and PH-Responsive Charge-Reversible Property. Macromol. Rapid Commun. 2020, 41 (15), 2000260.

92. Yu, Q.; Ding, Y.; Cao, H.; Lu, X.; Cai, Y. Use of Polyion Complexation for Polymerization-Induced Self-Assembly in Water under Visible Light Irradiation at $25^{\circ} \mathrm{C}$. ACS Macro Lett. 2015, 4 (11), 12931296.

93. Ding, Y.; Cai, M.; Cui, Z.; Huang, L.; Wang, L.; Lu, X.; Cai, Y. Synthesis of Low-Dimensional Polyion Complex Nanomaterials via Polymerization-Induced Electrostatic Self-Assembly. Angew. Chem. Int. Ed. 2017, 57 (4), 1053-1056.

94. Shen, L.; Li, Y.; Lu, Q.; Qi, X.; Wu, X.; Zhou, Z.; Shen, J. Directed Arrangement of SiRNA via Polymerization-Induced Electrostatic Self-Assembly. Chem. Commun. 2020, 56 (16), 2411-2414.

95. Wang, L.; Ding, Y.; Liu, Q.; Zhao, Q.; Dai, X.; Lu, X.; Cai, Y. Sequence-Controlled PolymerizationInduced Self-Assembly. ACS Macro Lett. 2019, 8 (5), 623-628.

96. Li, C.; Wang, Y.; Wang, X.; Gao, Z.; Ma, L.; Lu, X.; Cai, Y. Nanostructured Multiphase Condensation of Complex Coacervates in Polymerization-Induced Electrostatic Self-Assembly. ACS Macro Lett. 2021, 10 (7), 780-785.

97. Cai, M.; Ding, Y.; Wang, L.; Huang, L.; Lu, X.; Cai, Y. Synthesis of One-Component Nanostructured Polyion Complexes via Polymerization-Induced Electrostatic Self-Assembly. ACS Macro Lett. 2018, 7 (2), 208-212.

98. Huang, L.; Ding, Y.; Ma, Y.; Wang, L.; Liu, Q.; Lu, X.; Cai, Y. Colloidal Stable PIC Vesicles and Lamellae Enabled by Wavelength-Orthogonal Disulfide Exchange and Polymerization-Induced Electrostatic Self-Assembly. Macromolecules. 2019, 52 (12), 4703-4712.

99. Ding, Y.; Zhao, Q.; Wang, L.; Huang, L.; Liu, Q.; Lu, X.; Cai, Y. Polymerization-Induced SelfAssembly Promoted by Liquid-Liquid Phase Separation. ACS Macro Lett. 2019, 8 (8), 943-946.

100. Wang, Y.; Li, C.; Ma, L.; Wang, X.; Wang, K.; Lu, X.; Cai, Y. Interfacial Liquid-Liquid Phase Separation-Driven Polymerization-Induced Electrostatic Self-Assembly. Macromolecules. 2021, 54 (12), 5577-5585.

101. Zhao, Q.; Liu, Q.; Li, C.; Cao, L.; Ma, L.; Wang, X.; Cai, Y. Noncovalent Structural Locking of Thermoresponsive Polyion Complex Micelles, Nanowires, and Vesicles via Polymerization-Induced Electrostatic Self-Assembly Using an Arginine-like Monomer. Chem. Commun. 2020, 56 (36), 49544957.

102. Blanazs, A.; Madsen, J.; Battaglia, G.; Ryan, A. J.; Armes, S. P. Mechanistic Insights for Block Copolymer Morphologies: How Do Worms Form Vesicles? J. Am. Chem. Soc. 2011, 133 (41), $16581-16587$. 
103. Lovett, J. R.; Warren, N. J.; Armes, S. P.; Smallridge, M. J.; Cracknell, R. B. Order-Order Morphological Transitions for Dual Stimulus Responsive Diblock Copolymer Vesicles. Macromolecules. 2016, 49 (3), 1016-1025.

104. Blanazs, A.; Verber, R.; Mykhaylyk, O. O.; Ryan, A. J.; Heath, J. Z.; Douglas, C. W. I.; Armes, S. P. Sterilizable Gels from Thermoresponsive Block Copolymer Worms. J. Am. Chem. Soc. 2012, 134 (23), 9741-9748.

105. Mable, C. J.; Gibson, R. R.; Prevost, S.; McKenzie, B. E.; Mykhaylyk, O. O.; Armes, S. P. Loading of Silica Nanoparticles in Block Copolymer Vesicles during Polymerization-Induced Self-Assembly: Encapsulation Efficiency and Thermally Triggered Release. J. Am. Chem. Soc. 2015, 137 (51), 16098-16108.

106. Canton, I.; Warren, N. J.; Chahal, A.; Amps, K.; Wood, A.; Weightman, R.; Wang, E.; Moore, H.; Armes, S. P. Mucin-Inspired Thermoresponsive Synthetic Hydrogels Induce Stasis in Human Pluripotent Stem Cells and Human Embryos. ACS Cent. Sci. 2016, 2 (2), 65-74.

107. Mitchell, D. E.; Lovett, J. R.; Armes, S. P.; Gibson, M. I. Combining Biomimetic Block Copolymer Worms with an Ice-Inhibiting Polymer for the Solvent-Free Cryopreservation of Red Blood Cells. Angew. Chem. Int. Ed. 2016, 128 (8), 2851-2854.

108. Ladmiral, V.; Semsarilar, M.; Canton, I.; Armes, S. P. Polymerization-Induced Self-Assembly of Galactose-Functionalized Biocompatible Diblock Copolymers for Intracellular Delivery. J. Am. Chem. Soc. 2013, 135 (36), 13574-13581.

109. Mable, C. J.; Canton, I.; Mykhaylyk, O. O.; Gul, B. U.; Chambon, P.; Themistou, E.; Armes, S. P. Targeting Triple-Negative Breast Cancer Cells Using Dengue Virus-Mimicking PH-Responsive Framboidal Triblock Copolymer Vesicles. Chem. Sci. 2019, 10 (18), 4811-4821.

110. Dao, T. P. T.; Vezenkov, L.; Subra, G.; Amblard, M.; In, M.; Le Meins, J.-F.; Aubrit, F.; Moradi, M.A.; Ladmiral, V.; Semsarilar, M. Self-Assembling Peptide-Polymer Nano-Objects via Polymerization-Induced Self-Assembly. Macromolecules. 2020, 53 (16), 7034-7043.

111. Tuyen Dao, T. P.; Vezenkov, L.; Subra, G.; Ladmiral, V.; Semsarilar, M. Nano-Assemblies with CoreForming Hydrophobic Polypeptide via Polymerization-Induced Self-Assembly (PISA). Polym. Chem. 2021, 12 (1), 113-121.

112. Semsarilar, M.; Jones, E. R.; Blanazs, A.; Armes, S. P. Efficient Synthesis of Sterically-Stabilized Nano-Objects via RAFT Dispersion Polymerization of Benzyl Methacrylate in Alcoholic Media. Adv. Mater. 2012, 24 (25), 3378-3382.

113. Sugihara, S.; Armes, S. P.; Blanazs, A.; Lewis, A. L. Non-Spherical Morphologies from Cross-Linked Biomimetic Diblock Copolymers Using RAFT Aqueous Dispersion Polymerization. Soft Matter. 2011, 7 (22), 10787-10793.

114. Noy, J.-M.; Cao, C.; Stenzel, M. Length of the Stabilizing Zwitterionic Poly(2-Methacryloyloxyethyl Phosphorycholine) Block Influences the Activity of the Conjugated Arsenic Drug in Drug-Directed Polymerization-Induced Self-Assembly Particles. ACS Macro Lett. 2019, 8 (1), 57-63.

115. Cao, C.; Chen, F.; Garvey, C. J.; Stenzel, M. H. Drug-Directed Morphology Changes in Polymerization-Induced Self-Assembly (PISA) Influence the Biological Behavior of Nanoparticles. 
ACS Appl. Mater. Interfaces. 2020, 12 (27), 30221-30233.

116. Noy, J.-M.; Chen, F.; Stenzel, M. Post-Functionalization of Drug-Loaded Nanoparticles Prepared by Polymerization-Induced Self-Assembly (PISA) with Mitochondria Targeting Ligands. Beilstein J. Org. Chem. 2021, 17, 2302-2314.

117. Poon, C. K.; Tang, O.; Chen, X.-M.; Kim, B.; Hartlieb, M.; Pollock, C. A.; Hawkett, B. S.; Perrier, S. Fluorescent Labeling and Biodistribution of Latex Nanoparticles Formed by Surfactant-Free RAFT Emulsion Polymerization. Macromol. Biosci. 2017, 17 (10), 1600366.

118. Sobotta, F. H.; Hausig, F.; Harz, D. O.; Hoeppener, S.; Schubert, U. S.; Brendel, J. C. OxidationResponsive Micelles by a One-Pot Polymerization-Induced Self-Assembly Approach. Polym. Chem. 2018, 9 (13), 1593-1602.

119. Sobotta, F. H.; Kuchenbrod, M. T.; Gruschwitz, F. V.; Festag, G.; Bellstedt, P.; Hoeppener, S.; Brendel, J. C. Tuneable Time Delay in the Burst Release from Oxidation Sensitive Polymersomes Made by PISA. Angew. Chem. Int. Ed. 2021, 60 (46), 24716-24723.

120. Georgiou, P. G.; Marton, H. L.; Baker, A. N.; Congdon, T. R.; Whale, T. F.; Gibson, M. I. Polymer Self-Assembly Induced Enhancement of Ice Recrystallization Inhibition. J. Am. Chem. Soc. 2021, 143 (19), 7449-7461.

121. Zhou, W.; Qu, Q.; Xu, Y.; An, Z. Aqueous Polymerization-Induced Self-Assembly for the Synthesis of Ketone-Functionalized Nano-Objects with Low Polydispersity. ACS Macro Lett. 2015, 4 (5), 495499.

122. Byard, S. J.; Williams, M.; McKenzie, B. E.; Blanazs, A.; Armes, S. P. Preparation and Cross-Linking of All-Acrylamide Diblock Copolymer Nano-Objects via Polymerization-Induced Self-Assembly in Aqueous Solution. Macromolecules. 2017, 50 (4), 1482-1493.

123. Sun, H.; Cao, W.; Zang, N.; Clemons, T. D.; Scheutz, G. M.; Hu, Z.; Thompson, M. P.; Liang, Y.; Vratsanos, M.; Zhou, X.; Choi, W.; Sumerlin, B. S.; Stupp, S. I.; Gianneschi, N. C. Proapoptotic Peptide Brush Polymer Nanoparticles via Photoinitiated Polymerization-Induced Self-Assembly. Angew. Chem. 2020, 132 (43), 19298-19304.

124. You, Y.-Z.; Manickam, D. S.; Zhou, Q.-H.; Oupický, D. Reducible Poly(2-Dimethylaminoethyl Methacrylate): Synthesis, Cytotoxicity, and Gene Delivery Activity. J. Control. Release. 2007, 122 (3), 217-225.

125. van de Wetering, P.; Cherng, J.-Y.; Talsma, H.; Hennink, W. E. Relation between Transfection Efficiency and Cytotoxicity of Poly(2-(Dimethylamino)Ethyl Methacrylate)/Plasmid Complexes. $J$. Control. Release. 1997, 49 (1), 59-69.

126. Qiu, L.; Xu, C.-R.; Zhong, F.; Hong, C.-Y.; Pan, C.-Y. Fabrication of Functional Nano-Objects through RAFT Dispersion Polymerization and Influences of Morphology on Drug Delivery. ACS Appl. Mater. Interfaces. 2016, 8 (28), 18347-18359.

127. Engström, J.; Asem, H.; Brismar, H.; Zhang, Y.; Malkoch, M.; Malmström, E. In Situ Encapsulation of Nile Red or Doxorubicin during RAFT-Mediated Emulsion Polymerization via PolymerizationInduced Self-Assembly for Biomedical Applications. Macromol. Chem. Phys. 2020, 221 (5), 1900443. 
128. Khor, S. Y.; Vu, M. N.; Pilkington, E. H.; Johnston, A. P. R.; Whittaker, M. R.; Quinn, J. F.; Truong, N. P.; Davis, T. P. Elucidating the Influences of Size, Surface Chemistry, and Dynamic Flow on Cellular Association of Nanoparticles Made by Polymerization-Induced Self-Assembly. Small. 2018, 14 (34), 1801702.

129. Gurnani, P.; Bray, C. P.; Richardson, R. A. E.; Peltier, R.; Perrier, S. Heparin-Mimicking Sulfonated Polymer Nanoparticles via RAFT Polymerization-Induced Self-Assembly. Macromol. Rapid Commun. 2019, 40 (2), 1800314.

130. Hoogenboom, R. Poly(2-Oxazoline)s: A Polymer Class with Numerous Potential Applications. Angew. Chem. Int. Ed. 2009, 48 (43), 7978-7994.

131. Le, D.; Wagner, F.; Takamiya, M.; Hsiao, I.-L.; Gil Alvaradejo, G.; Strähle, U.; Weiss, C.; Delaittre, G. Straightforward Access to Biocompatible Poly(2-Oxazoline)-Coated Nanomaterials by Polymerization-Induced Self-Assembly. Chem. Commun. 2019, 55 (26), 3741-3744.

132. Varlas, S.; Foster, J. C.; O’Reilly, R. K. Ring-Opening Metathesis Polymerization-Induced SelfAssembly (ROMPISA). Chem. Commun. 2019, 55 (62), 9066-9071.

133. Foster, J. C.; Varlas, S.; Blackman, L. D.; Arkinstall, L. A.; O’Reilly, R. K. Ring-Opening Metathesis Polymerization in Aqueous Media Using a Macroinitiator Approach. Angew. Chem. Int. Ed. 2018, 57 (33), 10672-10676.

134. Le, D.; Dilger, M.; Pertici, V.; Diabaté, S.; Gigmes, D.; Weiss, C.; Delaittre, G. Ultra-Fast Synthesis of Multivalent Radical Nanoparticles by Ring-Opening Metathesis Polymerization-Induced SelfAssembly. Angew. Chem. Int. Ed. 2019, 58 (14), 4725-4731.

135. Wright, D. B.; Proetto, M. T.; Touve, M. A.; Gianneschi, N. C. Ring-Opening Metathesis Polymerization-Induced Self-Assembly (ROMPISA) of a Cisplatin Analogue for High Drug-Loaded Nanoparticles. Polym. Chem. 2019, 10 (23), 2996-3000.

136. Varlas, S.; Maitland, G. L.; Derry, M. J. Protein-, (Poly)Peptide-, and Amino Acid-Based Nanostructures Prepared via Polymerization-Induced Self-Assembly. Polymers. 2021, 13 (16), 2603.

137. Six, J.-L.; Ferji, K. Polymerization Induced Self-Assembly: An Opportunity toward the SelfAssembly of Polysaccharide-Containing Copolymers into High-Order Morphologies. Polym. Chem. 2019, $10(1), 45-53$.

138. Ma, C.; Liu, X.; Wu, G.; Zhou, P.; Zhou, Y.; Wang, L.; Huang, X. Efficient Way to Generate ProteinBased Nanoparticles by in-Situ Photoinitiated Polymerization-Induced Self-Assembly. ACS Macro Lett. 2017, 6, 689-694.

139. Liu, X.; Gao, W. In Situ Growth of Self-Assembled Protein-Polymer Nanovesicles for Enhanced Intracellular Protein Delivery. ACS Appl. Mater. Interfaces. 2017, 9 (3), 2023-2028.

140. Rucco, D. J.; Barnes, B. E.; Garrison, J. B.; Sumerlin, B. S.; Savin, D. A. Modular Genetic Code Expansion Platform and PISA Yield Well-Defined Protein-Polymer Assemblies. Biomacromolecules. 2020, 21 (12), 5077-5085.

141. Chiang, C.-W.; Liu, X.; Sun, J.; Guo, J.; Tao, L.; Gao, W. Polymerization-Induced Coassembly of Enzyme-Polymer Conjugates into Comicelles with Tunable and Enhanced Cascade Activity. Nano Lett. 2020, 20 (2), 1383-1387. 
142. Yang, L.; Liang, M.; Cui, C.; Li, X.; Li, L.; Pan, X.; Yazd, H. S.; Hong, M.; Lu, J.; Cao, Y. C.; Tan, W. Enhancing the Nucleolytic Resistance and Bioactivity of Functional Nucleic Acids by Diverse Nanostructures through in Situ Polymerization-Induced Self-Assembly. ChemBioChem. 2021, 22 (4), 754-759.

143. Lückerath, T.; Koynov, K.; Loescher, S.; Whitfield, C. J.; Nuhn, L.; Walther, A.; Barner-Kowollik, C.; Ng, D. Y. W.; Weil, T. DNA-Polymer Nanostructures by RAFT Polymerization and Polymerization-Induced Self-Assembly. Angew. Chem. Int. Ed. 2020, 59 (36), 15474-15479.

144. Hatton, F. L.; Ruda, M.; Lansalot, M.; D’Agosto, F.; Malmström, E.; Carlmark, A. XyloglucanFunctional Latex Particles via RAFT-Mediated Emulsion Polymerization for the Biomimetic Modification of Cellulose. Biomacromolecules. 2016, 17 (4), 1414-1424.

145. Kapishon, V.; Whitney, R. A.; Champagne, P.; Cunningham, M. F.; Neufeld, R. J. Polymerization Induced Self-Assembly of Alginate Based Amphiphilic Graft Copolymers Synthesized by Single Electron Transfer Living Radical Polymerization. Biomacromolecules. 2015, 16 (7), 2040-2048.

146. Chaduc, I.; Reynaud, E.; Dumas, L.; Albertin, L.; D’Agosto, F.; Lansalot, M. From Well-Defined Poly(N-Acryloylmorpholine)-Stabilized Nanospheres to Uniform Mannuronan- and GuluronanDecorated Nanoparticles by RAFT Polymerization-Induced Self-Assembly. Polymer. 2016, 106, $218-228$.

147. Ikkene, D.; Arteni, A. A.; Ouldali, M.; Francius, G.; Brûlet, A.; Six, J.-L.; Ferji, K. Direct Access to Polysaccharide-Based Vesicles with a Tunable Membrane Thickness in a Large Concentration Window via Polymerization-Induced Self-Assembly. Biomacromolecules. 2021, 22 (7), 3128-3137.

148. Ferji, K.; Venturini, P.; Cleymand, F.; Chassenieux, C.; Six, J.-L. In Situ Glyco-Nanostructure Formulation via Photo-Polymerization Induced Self-Assembly. Polym. Chem. 2018, 9 (21), 28682872.

149. Elzoghby, A. O.; Samy, W. M.; Elgindy, N. A. Albumin-Based Nanoparticles as Potential Controlled Release Drug Delivery Systems. J. Control. Release. 2012, 157 (2), 168-182.

150. Li, J.; Mo, L.; Lu, C.-H.; Fu, T.; Yang, H.-H.; Tan, W. Functional Nucleic Acid-Based Hydrogels for Bioanalytical and Biomedical Applications. Chem. Soc. Rev. 2016, 45 (5), 1410-1431.

151. Petzetakis, N.; Dove, A. P.; O’Reilly, R. K. Cylindrical Micelles from the Living CrystallizationDriven Self-Assembly of Poly(Lactide)-Containing Block Copolymers. Chem Sci. 2011, 2 (5), 955960.

152. Petzetakis, N.; Walker, D.; Dove, A. P.; O’Reilly, R. K. Crystallization-Driven Sphere-to-Rod Transition of Poly(Lactide)-b-Poly(Acrylic Acid) Diblock Copolymers: Mechanism and Kinetics. Soft Matter. 2012, 8 (28), 7408.

153. Petzetakis, N.; Robin, M. P.; Patterson, J. P.; Kelley, E. G.; Cotanda, P.; Bomans, P. H. H.; Sommerdijk, N. A. J. M.; Dove, A. P.; Epps, T. H.; O’Reilly, R. K. Hollow Block Copolymer Nanoparticles through a Spontaneous One-Step Structural Reorganization. ACS Nano. 2013, 7 (2), $1120-1128$.

154. Li, Z.; Sun, L.; Zhang, Y.; Dove, A. P.; O’Reilly, R. K.; Chen, G. Shape Effect of Glyco-Nanoparticles on Macrophage Cellular Uptake and Immune Response. ACS Macro Lett. 2016, 5 (9), 1059-1064. 
155. Li, Z.; Zhang, Y.; Wu, L.; Yu, W.; Wilks, T. R.; Dove, A. P.; Ding, H.; O’Reilly, R. K.; Chen, G.; Jiang, M. Glyco-Platelets with Controlled Morphologies via Crystallization-Driven Self-Assembly and Their Shape-Dependent Interplay with Macrophages. ACS Macro Lett. 2019, 596-602.

156. He, X.; He, Y.; Hsiao, M.-S.; Harniman, R. L.; Pearce, S.; Winnik, M. A.; Manners, I. Complex and Hierarchical 2D Assemblies via Crystallization-Driven Self-Assembly of Poly(l-Lactide) Homopolymers with Charged Termini. J. Am. Chem. Soc. 2017, 139 (27), 9221-9228.

157. Song, Y.; Chen, Y.; Su, L.; Li, R.; Letteri, R. A.; Wooley, K. L. Crystallization-Driven Assembly of Fully Degradable, Natural Product-Based Poly(1-Lactide)-Block-Poly( $\alpha$-d-Glucose Carbonate)s in Aqueous Solution. Polymer. 2017, 122, 270-279.

158. Pitto-Barry, A.; Kirby, N.; Dove, A. P.; O’Reilly, R. K. Expanding the Scope of the CrystallizationDriven Self-Assembly of Polylactide-Containing Polymers. Polym Chem. 2014, 5 (4), 1427-1436.

159. Inam, M.; Foster, J. C.; Gao, J.; Hong, Y.; Du, J.; Dove, A. P.; O’Reilly, R. K. Size and Shape Affects the Antimicrobial Activity of Quaternized Nanoparticles. J. Polym. Sci. Part Polym. Chem. 2019, 57 (3), 255-259.

160. Malikmammadov, E.; Tanir, T. E.; Kiziltay, A.; Hasirci, V.; Hasirci, N. PCL and PCL-Based Materials in Biomedical Applications. J. Biomater. Sci. Polym. Ed. 2018, 29 (7-9), 863-893.

161. Zhu, W.; Peng, B.; Wang, J.; Zhang, K.; Liu, L.; Chen, Y. Bamboo Leaf-Like Micro-Nano Sheets Self-Assembled by Block Copolymers as Wafers for Cells. Macromol. Biosci. 2014, 14 (12), 1764 1770.

162. Ganda, S.; Dulle, M.; Drechsler, M.; Förster, B.; Förster, S.; Stenzel, M. H. Two-Dimensional SelfAssembled Structures of Highly Ordered Bioactive Crystalline-Based Block Copolymers. Macromolecules. 2017, 50 (21), 8544-8553.

163. Ganda, S.; Jiang, Y.; Thomas, D. S.; Eliezar, J.; Stenzel, M. H. Biodegradable Glycopolymeric Micelles Obtained by RAFT-Controlled Radical Ring-Opening Polymerization. Macromolecules. 2016, 49 (11), 4136-4146.

164. Arno, M. C.; Inam, M.; Coe, Z.; Cambridge, G.; Macdougall, L. J.; Keogh, R.; Dove, A. P.; O’Reilly, R. K. Precision Epitaxy for Aqueous 1D and 2D Poly( $\varepsilon$-Caprolactone) Assemblies. J. Am. Chem. Soc. 2017, 139 (46), 16980-16985.

165. Yu, W.; Foster, J. C.; Dove, A. P.; O’Reilly, R. K. Length Control of Biodegradable Fiber-Like Micelles via Tuning Solubility: A Self-Seeding Crystallization-Driven Self-Assembly of Poly( $\varepsilon$ Caprolactone)-Containing Triblock Copolymers. Macromolecules. 2020, 53 (4), 1514-1521.

166. Tong, Z.; Su, Y.; Jiang, Y.; Xie, Y.; Chen, S.; O’Reilly, R. K. Spatially Restricted Templated Growth of Poly( $\varepsilon$-Caprolactone) from Carbon Nanotubes by Crystallization-Driven Self-Assembly. Macromolecules. 2021, 54 (6), 2844-2851.

167. Ganda, S.; Wong, C. K.; Stenzel, M. H. Corona-Loading Strategies for Crystalline Particles Made by Living Crystallization-Driven Self-Assembly. Macromolecules. 2021, 54 (14), 6662-6669.

168. Finnegan, J. R.; He, X.; Street, S. T. G.; Garcia-Hernandez, J. D.; Hayward, D. W.; Harniman, R. L.; Richardson, R. M.; Whittell, G. R.; Manners, I. Extending the Scope of "Living" CrystallizationDriven Self-Assembly: Well-Defined 1D Micelles and Block Comicelles from Crystallizable 
Polycarbonate Block Copolymers. J. Am. Chem. Soc. 2018, 140 (49), 17127-17140.

169. Kang, L.; Chao, A.; Zhang, M.; Yu, T.; Wang, J.; Wang, Q.; Yu, H.; Jiang, N.; Zhang, D. Modulating the Molecular Geometry and Solution Self-Assembly of Amphiphilic Polypeptoid Block Copolymers by Side Chain Branching Pattern. J. Am. Chem. Soc. 2021, 143 (15), 5890-5902.

170. Jiang, N.; Yu, T.; Darvish, O. A.; Qian, S.; Mkam Tsengam, I. K.; John, V.; Zhang, D. CrystallizationDriven Self-Assembly of Coil-Comb-Shaped Polypeptoid Block Copolymers: Solution Morphology and Self-Assembly Pathways. Macromolecules. 2019, 52 (22), 8867-8877.

171. Shi, Z.; Wei, Y.; Zhu, C.; Sun, J.; Li, Z. Crystallization-Driven Two-Dimensional Nanosheet from Hierarchical Self-Assembly of Polypeptoid-Based Diblock Copolymers. Macromolecules. 2018, 51 (16), 6344-6351.

172. Wei, Y.; Tian, J.; Zhang, Z.; Zhu, C.; Sun, J.; Li, Z. Supramolecular Nanosheets Assembled from Poly(Ethylene Glycol)-b-Poly(N-(2-Phenylethyl)Glycine) Diblock Copolymer Containing Crystallizable Hydrophobic Polypeptoid: Crystallization Driven Assembly Transition from Filaments to Nanosheets. Macromolecules. 2019, 52 (4), 1546-1556.

173. Wei, Y.; Liu, F.; Li, M.; Li, Z.; Sun, J. Dimension Control on Self-Assembly of a Crystalline CoreForming Polypeptoid Block Copolymer: 1D Nanofibers versus 2D Nanosheets. Polym. Chem. 2021, $12(8), 1147-1154$.

174. Xuan, S.; Jiang, X.; Balsara, N. P.; Zuckermann, R. N. Crystallization and Self-Assembly of ShapeComplementary Sequence-Defined Peptoids. Polym. Chem. 2021, 12 (33), 4770-4777.

175. Wang, Z.; Lin, M.; Bonduelle, C.; Li, R.; Shi, Z.; Zhu, C.; Lecommandoux, S.; Li, Z.; Sun, J. Thermoinduced Crystallization-Driven Self-Assembly of Bioinspired Block Copolymers in Aqueous Solution. Biomacromolecules. 2020.

176. Legros, C.; De Pauw-Gillet, M.-C.; Tam, K. C.; Taton, D.; Lecommandoux, S. Crystallisation-Driven Self-Assembly of Poly(2-Isopropyl-2-Oxazoline)-Block-Poly(2-Methyl-2-Oxazoline) above the LCST. Soft Matter. 2015, 11 (17), 3354-3359.

177. Finnegan, J.; Pilkington, E.; Alt, K.; Rahim, M. A.; Kent, S. J.; Davis, T. P.; Kempe, K. Stealth Nanorods via the Aqueous Living Crystallisation-Driven Self-Assembly of Poly(2-Oxazoline)s. Chem. Sci. 2021.

178. Mees, M. A.; Hoogenboom, R. Full and Partial Hydrolysis of Poly(2-Oxazoline)s and the Subsequent Post-Polymerization Modification of the Resulting Polyethylenimine (Co)Polymers. Polym. Chem. 2018, 9 (40), 4968-4978.

179. Luxenhofer, R.; Han, Y.; Schulz, A.; Tong, J.; He, Z.; Kabanov, A. V.; Jordan, R. Poly(2-Oxazoline)s as Polymer Therapeutics. Macromol. Rapid Commun. 2012, 33 (19), 1613-1631.

180. Nabiyan, A.; Biehl, P.; Schacher, F. H. Crystallization vs Metal Chelation: Solution Self-Assembly of Dual Responsive Block Copolymers. Macromolecules. 2020, 53 (13), 5056-5067.

181. Volkmann, L.; Köhler, M.; Sobotta, F. H.; Enke, M. T.; Brendel, J. C.; Schacher, F. H. Poly(2Acrylamidoglycolic Acid) (PAGA): Controlled Polymerization Using RAFT and Chelation of Metal Cations. Macromolecules. 2018, 51 (18), 7284-7294. 
182. Yu, Q.; Roberts, M. G.; Pearce, S.; Oliver, A. M.; Zhou, H.; Allen, C.; Manners, I.; Winnik, M. A. Rodlike Block Copolymer Micelles of Controlled Length in Water Designed for Biomedical Applications. Macromolecules. 2019, 52 (14), 5231-5244.

183. Yu, Q.; G. Roberts, M.; Houdaihed, L.; Liu, Y.; Ho, K.; Walker, G.; Allen, C.; M. Reilly, R.; Manners, I.; A. Winnik, M. Investigating the Influence of Block Copolymer Micelle Length on Cellular Uptake and Penetration in a Multicellular Tumor Spheroid Model. Nanoscale. 2021, 13 (1), 280-291.

184. Street, S. T. G.; He, Y.; Jin, X.-H.; Hodgson, L.; Verkade, P.; Manners, I. Cellular Uptake and Targeting of Low Dispersity, Dual Emissive, Segmented Block Copolymer Nanofibers. Chem. Sci. 2020, 11 (32), 8394-8408.

185. Wong, C. K.; Chen, F.; Walther, A.; Stenzel, M. H. Bioactive Patchy Nanoparticles with Compartmentalized Cargoes for Simultaneous and Trackable Delivery. Angew. Chem. Int. Ed. 2019, 58 (22), 7335-7340.

186. McInnes, S. J.; Voelcker, N. H. Silicon-Polymer Hybrid Materials for Drug Delivery. Future Med. Chem. 2009, 1 (6), 1051-1074. 